Ricardo Cesare Román Amigo

\title{
Otimização e Fabricação de Dispositivos Piezelétricos com Gradação Funcional de Material
}


Ricardo Cesare Román Amigo

\section{Otimização e Fabricação de Dispositivos Piezelétricos com Gradação Funcional de Material}

Dissertação apresentada à Escola Politécnica da Universidade de São Paulo para obtenção do Título de Mestre em Engenharia Mecânica. 


\title{
Ricardo Cesare Román Amigo
}

\section{Otimização e Fabricação de Dispositivos Piezelétricos com Gradação Funcional de Material}

\author{
Dissertação apresentada à Escola Politécnica \\ da Universidade de São Paulo para obtenção \\ do Título de Mestre em Engenharia Mecânica. \\ Área de concentração: \\ Engenharia de Controle e Automação \\ Mecânica \\ Orientador: \\ Prof. Dr. Emílio Carlos Nelli Silva
}


Este exemplar foi revisado e corrigido em relação à versão original, sob responsabilidade única do autor e com a anuência de seu orientador.

São Paulo, 18 de março de 2013.

Assinatura do autor

Assinatura do orientador

Amigo, Ricardo Cesare Román

Otimização e fabricação de dispositivos piezelétricos com gradação funcional de material / R.C.R. Amigo. -- versão corr. -São Paulo, 2013.

$91 \mathrm{p}$.

Dissertação (Mestrado) - Escola Politécnica da Universidade de São Paulo. Departamento de Engenharia Mecatrônica e de Sistemas Mecânicos.

1. Métodos topológicos (Otimização) 2. Atuadores piezelétricos 3. Sensores 4. Sinterização I. Universidade de São Paulo. Escola Politécnica. Departamento de Engenharia Mecatrônica e de Sistemas Mecânicos II. t. 


\section{Agradecimentos}

Ao orientador, Prof. Dr. Emílio Carlos Nelli Silva, por sua contagiante dedicação.

Ao Conselho Nacional de Desenvolvimento Científico e Tecnológico (CNPq) pelo apoio financeiro a este trabalho, através de bolsa de mestrado (Processo 558411/2010-0).

À Profa. Dra. Izabel Fernanda Machado, por me ajudar na prática.

Aos amigos do Laboratório de Ultrassom, Fausto, Marco, Nico, Timóteo e Wellington pela ajuda constante no desenvolvimento deste trabalho.

Aos otimizadores Bencho, César, Douglas, Flávio, Lance, Luís, Ronny, Sandro e Teves, por serem os únicos que sabiam do que eu estava falando em boa parte destes últimos anos.

Aos vizinhos de laboratório, Barazani, Chaves, Erick, João e Lucas, pelos momentos de extensão acadêmica.

Ao Karatê Poli-USP, que tornou minha relação com a Escola Politécnica muito mais intensa.

À minha mãe e às minhas irmãs, Carolina e Cristina, por serem minha família.

Ao meu pai, que continua me inspirando a tentar deixar o mundo melhor do que eu recebi. 


\section{Resumo}

Cerâmicas piezelétricas possibilitam posicionamento e sensoriamento de precisão ou captação de energia mecânica valendo-se do efeito piezelétrico, capaz de converter energia mecânica em elétrica ou o contrário. Para aprimorar ou estender as aplicações dessas cerâmicas, mecanismos flexíveis podem ser acoplados a elas, formando um Dispositivos Piezelétricos Flextensionais (DPF). No projeto desse tipo de estrutura, o conceito de Material com Gradação Funcional (MGF) é interessante, já que esses materiais apresentam variações graduais de suas propriedades efetivas, permitindo a alternância entre um material mais flexível e um mais rígido de acordo com a intensidade de deslocamento desejada em cada região da estrutura. Assim, neste trabalho, implementa-se o Método de Otimização Topológica (MOT) no projeto de estruturas gradadas com o intuito de identificar as vantagens e desvantagens da utilização do conceito de MGF em DPF. Esse método combina algoritmos de otimização e o Método dos Elementos Finitos (MEF) para distribuir material dentro de um domínio fixo através de um modelo de material, que no caso estudado é o de Material Isotrópico Sólido com Penalização (MISP) adaptado a MGF. Na fabricação desses dispositivos otimizados, utiliza-se a Sinterização por Jato de Plasma (SJP) para a obtenção de tarugos gradados que são submetidos a processos de eletro-erosão e de corte a laser. Por fim, para a verificação dos resultados numéricos, utiliza-se um vibrômetro para aferir os deslocamentos dos protótipos de atuadores fabricados. Os resultados demonstram que o MOT é adequado ao projeto de dispositivos piezelétricos gradados e que, pela sua implementação, mecanismos com gradação funcional de material apresentam ganhos significativos em desempenho quando comparados aos análogos constituídos por materiais homogêneos. Além disso, o processo de fabricação proposto se mostra viável, possibilitando a obtenção de protótipos que se comportam conforme esperado.

Palavras-chave: Atuadores Piezelétricos. Materiais com Gradação Funcional. Método de Otimização Topológica. Sinterização por Jato de Plasma. 


\section{Abstract}

Piezoelectric devices enable precision positioning and sensing or mechanical energy harvesting based on the piezoelectric effect. In flextensional piezoelectric devices, flexible coupling structures are attached to ceramics to improve or extend the application possibilities. On the design of this kind of structure, the concept of Functionally Graded Materials (FGM) can be interesting, since it allows gradual variations of its effective properties along some direction by mixing two or more materials. Thus, in order to identify the advantages and disadvantages of using FGM, graded flexible coupling structures that maximize the performance of piezoelectric devices are obtained by implementing the Topology Optimization Method (TOM). This method combines optimization algorithms and the Finite Element Method (FEM) to distribute material inside a fixed domain. In this work, the formulation is based on the Solid Isotropic Material with Penalization (SIMP) material model adapted for the FGM concept, which can represent continuous change in material properties along the domain. Resulting optimal graded topologies of coupling structures are presented and compared with homogeneous structures. Finally, graded devices are manufactured through Spark Plasma Sintering (SPS) technique in order to be characterized, validating numerical results. The numerical results demonstrate the TOM efficacy in designing functionally graded piezoelectric devices and show, by its implementation, significant gains in graded mechanisms performance when compared with analogous homogeneous. Furthermore, the feasibility of proposed manufacturing process is confirmed, allowing the fabrication of prototypes with expected behavior.

Keywords: Piezoelectric Actuators. Functionally Graded Material. Topology Optimization Method. Spark Plasma Sintering. 


\section{Sumário}

\section{Lista de Figuras}

\section{Lista de Tabelas}

\section{Lista de Abreviaturas}

\section{Lista de Símbolos}

1 Introdução 1

1.1 Dispositivos Piezelétricos . . . . . . . . . . . . . . . . . . . . 1

1.1.1 Aplicações de Dispositivos Piezelétricos . . . . . . . . . . . . . . . . . 1

1.1.2 Projeto de Dispositivos Piezelétricos com Gradação Funcional de Material ....................... . . . 2

1.1.3 Fabricação de Peças com Gradação Funcional de Material . . . . . . 4

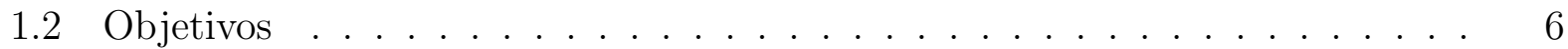

1.3 Justificativa . . . . . . . . . . . . . . . . . . . . . . . . 7

1.4 Organização do Texto . . . . . . . . . . . . . . . . . . . . 7

2 Projeto de Dispositivos Piezelétricos Gradados $\quad 8$

2.1 Métodos de Otimização Estrutural . . . . . . . . . . . . . . . . . . . 8

2.2 Método de Otimização Topológica . . . . . . . . . . . . . . . . . . . . . . 10

2.2 .1 Modelo de Material . . . . . . . . . . . . . . . . . . . . . 10

2.2 .2 Escala de Cinza . . . . . . . . . . . . . . . . . . . . . . 11

2.2.3 Instabilidade de Tabuleiro e Dependência de Malha . . . . . . . . . . 12

2.3 Projeto de Dispositivos com Gradação Funcional de Material . . . . . . . . . 14

2.3.1 Projeto de Atuadores Piezelétricos . . . . . . . . . . . . . 15 
2.3.2 Projeto de Sensores Piezelétricos . . . . . . . . . . . . . . 16

3 Implementação Numérica $\quad 18$

3.1 Aspectos Numéricos da Implementação do MOT . . . . . . . . . . . . . . . . 18

3.1 .1 Aproximação Contínua da Distribuição de Material . . . . . . . . . . 18

3.1 .2 Linearização das Funções-Objetivo e das Restrições . . . . . . . . . . 18

3.2 Procedimento de Otimização . . . . . . . . . . . . . . . . . . . . . . . 19

3.3 Otimização de Atuadores Piezelétricos . . . . . . . . . . . . . . . . . . . 20

3.3.1 Formulação Discreta . . . . . . . . . . . . . . . . . . . . 20

3.3.2 Análise de Sensibilidades . . . . . . . . . . . . . . . . . . . . . . . 21

3.4 Otimização de Sensores Piezelétricos . . . . . . . . . . . . . . . . . . 23

3.4.1 Formulação Discreta . . . . . . . . . . . . . . . . . . . 23

3.4 .2 Análise de Sensibilidades . . . . . . . . . . . . . . . . . . . . . . 24

4 Fabricação, Caracterização e Verificação Experimental 25

4.1 Sequência de Atividades para a fabricação de dispositivos piezelétricos gradados 25

4.2 Método Ultrassônico de Caracterização de Materiais . . . . . . . . . . . . . . 26

4.3 Estimativa das Propriedades Elásticas de Gradações Funcionais . . . . . . . 28

4.4 Processo de Fabricação de Dispositivos com Gradação Funcional de Material 30

4.5 Método de Verificação Experimental de Atuadores Piezelétricos . . . . . . . . 32

5 Resultados Numéricos e Discussões $\quad 34$

5.1 Moonie Piezelétrico . . . . . . . . . . . . . . . . . . . . . . . . 34

5.1.1 Otimização com Restrições de Volume e Manufatura . . . . . . . . . 34

5.1 .2 Otimização sem Restrições de Volume e Manufatura . . . . . . . . . . 37

5.1 .3 Discussão . . . . . . . . . . . . . . . . . . . . 40

5.2 Garra Piezelétrica . . . . . . . . . . . . . . . . . . . . . 41

5.2.1 Otimização com Restrições de Volume e Manufatura . . . . . . . . . 41

5.2.2 Otimização sem Restrições de Volume e Manufatura . . . . . . . . . . . 44 
5.2 .3 Discussão . . . . . . . . . . . . . . . . . . . . . . . 47

5.3 Sensor de Força Piezelétrico . . . . . . . . . . . . . . . . . . . . . 47

5.3.1 Otimização com Restrições de Volume e Manufatura ....... . 49

5.3.2 Otimização sem Restrições de Volume e Manufatura . . . . . . . . . . 50

5.3 .3 Discussão . . . . . . . . . . . . . . . . . . . . . 54

6 Resultados Experimentais e Discussões $\quad 57$

6.1 Protótipos com Formas Arbitrárias . . . . . . . . . . . . . . . . 57

6.1.1 Fabricação . . . . . . . . . . . . . . . . . . 57

6.1 .2 Discussão . . . . . . . . . . . . . . . . . . . . . 58

6.2 Gradação Cobre-Níquel . . . . . . . . . . . . . . . . . . . . . 58

6.2 .1 Fabricação . . . . . . . . . . . . . . . . . . . . . . 59

6.2 .2 Caracterização . . . . . . . . . . . . . . . 60

6.2 .3 Discussão . . . . . . . . . . . . . . . . . . . . . . . 60

6.3 Protótipo de Atuador Piezelétrico . . . . . . . . . . . . . . . . 61

6.3.1 Fabricação . . . . . . . . . . ......... 61

6.3.2 Verificação Experimental . . . . . . . . . . . . . . . 63

6.3 .3 Discussão . . . . . . . . . . . . . . . . . . . . . . 65

7 Conclusões e Trabalhos Futuros $\quad 67$

7.1 Conclusões . . . . . . . . . . . . . . . . . . . . . . . . . 67

7.2 Trabalhos Futuros . . . . . . . . . . . . . . . . . . 68

$\begin{array}{ll}\text { Referências } & 69\end{array}$

$\begin{array}{ll}\text { Apêndice A - Piezeletricidade } & 75\end{array}$

Apêndice B - Método dos Elementos Finitos Piezelétrico 78

B.1 Princípio Variacional Piezelétrico . . . . . . . . . . . . . . . . . . 78

B.2 Estado Plano de Tensões Mecânicas . . . . . . . . . . . . . . . . . . . . 80 
B.3 Elemento Isoparamétrico de Quatro Nós . . . . . . . . . . . . . . . . . . 81

B.4 Formulação Matricial do Elemento . . . . . . . . . . . . . . . . . . 82

B.5 Prescrição de Deslocamentos . . . . . . . . . . . . . . . . . . . . . . 83

B.6 Verificação da Implementação do MEF . . . . . . . . . . . . . . . . . . . . . 84

Apêndice C - Programação Linear Sequencial $\quad 88$

$\begin{array}{ll}\text { Anexo A - Relações entre Constantes Elásticas } & 90\end{array}$

Anexo B - Propriedades Teóricas do Material Piezelétrico Utilizado 91 


\section{Lista de Figuras}

1.1 Princípio de funcionamento de (a) atuadores e (b) sensores piezelétricos flextensionais com gradação funcional de material em sua estrutura acoplada. 2

1.2 Microestrutura da seção transversal do tronco de um bambu (SILVA; WALTERS;

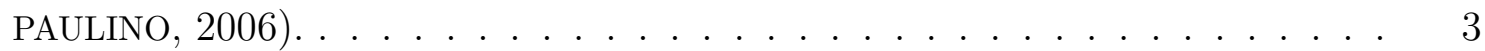

1.3 Microestrutura da gradação funcional entre um material A (cerâmico) e um material B (metálico) (KIEBACK; NEUBRAND; RIEDEL, 2003) . . . . . . . . . . 3

1.4 Vista em corte da montagem para SJP. . . . . . . . . . . . . . . . . 5

1.5 Sequência do desenvolvimento de dispositivos piezelétricos com gradação funcional de material. . . . . . . . . . . . . . . . . . . . . . 6

2.1 Classificação dos métodos de otimização estrutural. . . . . . . . . . . . . . 8

2.2 Exemplo de aplicação dos métodos de otimização (a) paramétrica, (b) de forma e $($ c) topológica. . . . . . . . . . . . . . . . . . . . . . . . 9 9

2.3 Ocorrência de escala de cinza $($ KIYONO, 2008) . . . . . . . . . . . . . . . 11

2.4 Demonstração do aumento de mínimos locais provocado pelo aumento dos penalizadores. . . . . . . . . . . . . . . . . . . . . 11

2.5 Ocorrência da instabilidade de tabuleiro (KIYONO, 2008) . . . . . . . . . . . . 12

2.6 Topologia ótima obtida com malhas de (a) 1200 elementos, (b) 1875 elementos e (c) 3000 elementos $($ KIYONO, 2008) . . . . . . . . . . . . . . . . 12

2.7 Raio de abrangência do filtro de projeção em (a) malha grossa e (b) malha fina. 13

2.8 Perfis da função peso de acordo com o valor de $g$. . . . . . . . . . . . . . . 14

2.9 Caso de carga para o cálculo do deslocamento estático gerado. . . . . . . . . 15

2.10 Caso de carga para o cálculo do potencial elétrico estático gerado. . . . . . . 16

3.1 Fluxograma do software de implementação do MOT. . . . . . . . . . . . . . 20

4.1 Sequência geral de projeto e fabricação de dispositivos piezelétricos gradados. 26 
4.2 Montagens para medição das velocidades de propagação de ondas (a) longitudinais e (b) de cisalhamento em uma amostra. . . . . . . . . . . . . 27

4.3 Goniômetro para caracterização ultrassônica de amostras de material. . . 27

4.4 Curvas limítrofes do módulo de compressibilidade $K$ e de cisalhamento $G$ em materiais compósitos com fases isotrópicas. . . . . . . . . . . . . . . . . . 29

4.5 Processo de fabricação de dispositivos piezelétricos gradados. . . . . . . . . . . 31

4.6 SPS-1050 (SPS SYNTEX INC., 2008) . . . . . . . . . . . . . . . . . . 31

4.7 Esquema de verificação experimental de protótipo via vibrômetro. . . . . . . . 32

4.8 Montagem para verificação experimental de protótipo via vibrômetro. . . . . 32

5.1 Domínio de projeto de moonie piezelétrico (dimensões em $\mathrm{mm}$ ) . . . . . . . . 35

5.2 Atuadores, sujeitos a restrições, com estrutura acoplada homogênea. . . . . . 36

5.3 Atuadores, sujeitos a restrições, com gradação funcional Cobre-Níquel restrita em camadas. . . . . . . . . . . . . . . . . . . . . . . . 37

5.4 Distribuição da gradação nos atuadores otimizados. . . . . . . . . . . . . . . 37

5.5 Deformada do atuador, sujeito a restrições, com gradação Cobre-Níquel em camadas perpendiculares. . . . . . . . . . . . . . . . . . . . . 38

5.6 Atuadores com estrutura acoplada homogênea. . . . . . . . . . . . . . . . . . 39

5.7 Atuador com gradação funcional livre de Cobre-Níquel. . . . . . . . . . . . . 39

5.8 Deformadas dos atuadores otimizados. . . . . . . . . . . . . . . . . 40

5.9 Domínio de projeto de garra piezelétrica (dimensões em $m m$ ). . . . . . . . . 42

5.10 Garras, sujeitas a restrições, com estrutura acoplada homogênea. . . . . . . . 43

5.11 Garras, sujeitas a restrições, com gradação funcional Cobre-Níquel restrita em camadas. . . . . . . . . . . . . . . . . . . . . . 44

5.12 Distribuição da gradação nas garras otimizadas. . . . . . . . . . . . . . . . . 44

5.13 Deformadas das garras otimizadas sujeitas a restrições. . . . . . . . . . . . 45

5.14 Garras com estrutura acoplada homogênea. . . . . . . . . . . . . . . . . 46

5.15 Garra com gradação funcional livre de Cobre-Níquel. . . . . . . . . . . . . . 47

5.16 Deformadas das garras otimizadas. . . . . . . . . . . . . . . . . . 48

5.17 Domínio de projeto de sensor de força piezelétrico (dimensões em $\mathrm{mm}$ ) . . . . 49 
5.18 Sensores, sujeitos a restrições, com estrutura acoplada homogênea. . . . . . . 50

5.19 Sensores, sujeitos a restrições, com gradação funcional Cobre-Níquel restrita em camadas. . . . . . . . . . . . . . . . . . . . . . . . 51

5.20 Distribuição da gradação nos sensores otimizados. . . . . . . . . . . . . . . . 51

5.21 Deformadas dos sensores otimizados sujeitos a restrições. . . . . . . . . . . . 52

5.22 Sensores com estrutura acoplada homogênea. . . . . . . . . . . . . . . . . . 53

5.23 Sensor com gradação funcional livre de Cobre-Níquel. . . . . . . . . . . . . . 54

5.24 Deformadas dos sensores otimizados. . . . . . . . . . . . . . . . 55

6.1 Curvas de temperatura e pressão mecânica da sinterização de amostras em Cobre e Níquel. . . . . . . . . . . . . . . . . . . . . . . . . . . . . . . 58

6.2 Eletroerosão de peça com gradação arbitrária. . . . . . . . . . . . . . . 58

6.3 Protótipos de dispositivos piezelétricos com topologia arbitrária. . . . . . . . 59

6.4 Seção da amostra de Cobre vista em microscópio óptico. . . . . . . . . . . . . 60

6.5 Peças obtidas em cada etapa do processo de fabricação. . . . . . . . . . . . 63

6.6 Protótipo de atuador gradado. . . . . . . . . . . . . . . . 63

6.7 Condição de ensaio do protótipo de atuador gradado. . . . . . . . . . . . 63

6.8 Deslocamento da face de atuação em função da frequência de excitação. . . . 64

6.9 Deformada (em $\mathrm{mm}$ ) do protótipo sob excitação de $18,2 \mathrm{~V}$ a $4 k H z$. . . . . 65

6.10 Variação máxima da rigidez conforme fração em volume de Níquel. . . . . . . 66

A.1 Estado dos dipolos elétricos (a) antes, (b) durante e (c) após o processo de polarização. . . . . . . . . . . . . . . . . . . . 75

A.2 Célula unitária de $\mathrm{BaTiO}_{3}$ (a) antes e (b) depois da polarização (CALLISTER,

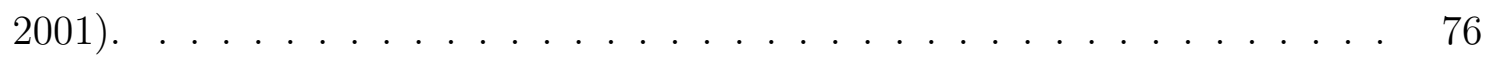

B.1 Estado Plano de Tensões Mecânicas. . . . . . . . . . . . . . . . . . . 80

B.2 Deformada do domínio controle obtida via COMSOL. . . . . . . . . . . . . 84

C.1 Programação linear sequencial e os limites móveis. . . . . . . . . . . . . . . . 89 


\section{Lista de Tabelas}

5.1 Pseudodensidades de gradação dos atuadores otimizados. . . . . . . . . . . . 36

5.2 Deslocamentos estáticos verticais gerados pelos atuadores otimizados sujeitos a restrições. . . . . . . . . . . . . . . . . . . . . . . . . . 38

5.3 Deslocamentos estáticos verticais gerados pelos atuadores otimizados. . . . . 40

5.4 Pseudodensidades de gradação das garras otimizadas sujeitas a restrições. . . 43

5.5 Deslocamentos estáticos horizontais médios de $\Gamma_{t}$ nas garras otimizadas sujeitas a restrições. . . . . . . . . . . . . . . . . . . . 45

5.6 Deslocamentos estáticos horizontais médios de $\Gamma_{t}$ nas garras otimizadas. . . . 47

5.7 Pseudodensidades de gradação dos sensores otimizados sujeitos a restrições. . 50

5.8 Potenciais elétricos no eletrodo $\Gamma_{\varphi}$ dos sensores otimizados sujeitos a restrições. 52

5.9 Deslocamentos verticais de $\Gamma_{t}$ nos sensores otimizados sujeitos a restrições. $\quad 52$

5.10 Potenciais elétricos no eletrodo $\Gamma_{\varphi}$ dos sensores otimizados. . . . . . . . . . 54

5.11 Deslocamentos verticais de $\Gamma_{t}$ nos sensores otimizados. . . . . . . . . . . 54

6.1 Características físicas das amostras homogêneas. . . . . . . . . . . . . . . . 59

6.2 Velocidades de propagação e constantes elásticas das amostras homogêneas. . 60

6.3 Determinação das frações em volume de Níquel a partir da rigidez desejada. 62

6.4 Massas de Cobre e Níquel em cada camada. . . . . . . . . . . . . . . . . . 62

6.5 Erro introduzido aos módulos de compressibilidade $(K)$ e de cisalhamento $(G)$ pela aproximação de material baseada no princípio variacional de Hashin-Shtrikman (HASHIN; SHTRIKMAN, 1963) . . . . . . . . . . . 66

B.1 Comparação entre deslocamentos na coordenada $x$ obtidos via AMIGOT e

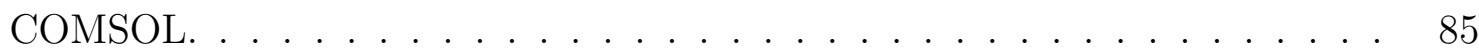

B.2 Comparação entre deslocamentos na coordenada $y$ obtidos via AMIGOT e

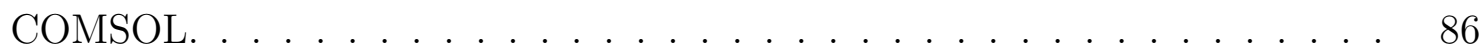

B.3 Comparação entre potenciais elétricos obtidos via AMIGOT e COMSOL. . 87 


\section{Lista de Abreviaturas}

ACDM Aproximação Contínua da Distribuição de Material

CEP Coletor de Energia Piezelétrico

CL Corte a Laser

CNC Controle Numérico Computadorizado

DPF Dispositivos Piezelétricos Flextensionais

DFE Domínio Fixo Estendido

EEF Eletro-Erosão a Fio

EPTM Estado Plano de Tensões Mecânicas

GDL Grau de Liberdade

MAC Modal Assurance Criterion

MC Método da Continuação

MDF Método de Diferenças Finitas

MEF Método dos Elementos Finitos

MEMS Sistemas Micro-eletromecânicos

MGF Material com Gradação Funcional

MISP Material Isotrópico Sólido com Penalização

MM Modelo de Material

MMA Método das Assíntotas Móveis

MOF Método de Otimização de Forma

MOP Método de Otimização Paramétrica

MOT Método de Otimização Topológica 
MOTAL Método de Otimização Topológica por Alocação de Lacunas

MOTCN Método de Otimização Topológica por Curvas de Nível

MOTE Método de Otimização Topológica Evolucionária

PL Programação Linear

PLS Programação Linear Sequencial

PZT Titanato Zircanato de Chumbo

SJP Sinterização por Jato de Plasma

TPGF Transdutores Piezelétricos com Gradação Funcional 


\title{
Lista de Símbolos
}

\author{
$A_{s} \quad$ amplitude de deslocamento \\ $A_{v} \quad$ amplitude de velocidade \\ $\mathbf{c}_{E} \quad$ matriz de constantes elásticas a campo elétrico constante \\ $d_{k_{i}} \quad$ variável de projeto da classe $k$ referente ao nó $i$ \\ $\mathbf{d}_{k} \quad$ vetor de variáveis de projeto da classe $k$ \\ $\mathbf{d}_{1}^{\min } \quad$ vetor de valores mínimos para variáveis de topologia (classe 1) \\ D vetor de deslocamentos elétricos \\ matriz de constantes piezelétricas \\ E módulo elástico de Young \\ E vetor de campos elétricos \\ $f \quad$ frequência \\ F vetor de carregamentos mecânicos \\ $\mathcal{F}() \quad$ função-multiobjetivo \\ G módulo elástico de cisalhamento \\ $K$ módulo elástico de compressibilidade \\ K matriz de rigidez \\ $L_{i}() \quad$ função-objetivo $i$ \\ M módulo elástico de onda de pressão \\ $p \quad$ coeficiente de penalização das variáveis de topologia no MISP \\ $q$ coeficiente de penalização das variáveis de gradação no MISP \\ Q vetor de carregamentos elétricos \\ $R_{i j} \quad$ distância entre os pontos $\mathbf{x}_{i}$ e $\mathbf{x}_{j}$ \\ $R_{\max } \quad$ raio de abrangência do filtro de projeção \\ $S_{i}^{r} \quad$ conjunto dos pontos compreendidos pela região $\Omega_{i}^{r}$ \\ S vetor de deformações mecânicas \\ T vetor de tensões mecânicas \\ U vetor de deslocamentos mecânicos \\ $v_{i}^{*} \quad$ aproximação da fração em volume da fase $i$ na mistura \\ $V_{e} \quad$ volume do elemento finito $e$ \\ $w_{i j} \quad$ peso de $d_{j}$ no cálculo de $\rho_{i}$ pela técnica de projeção \\ vetor de coordenadas de um ponto
}




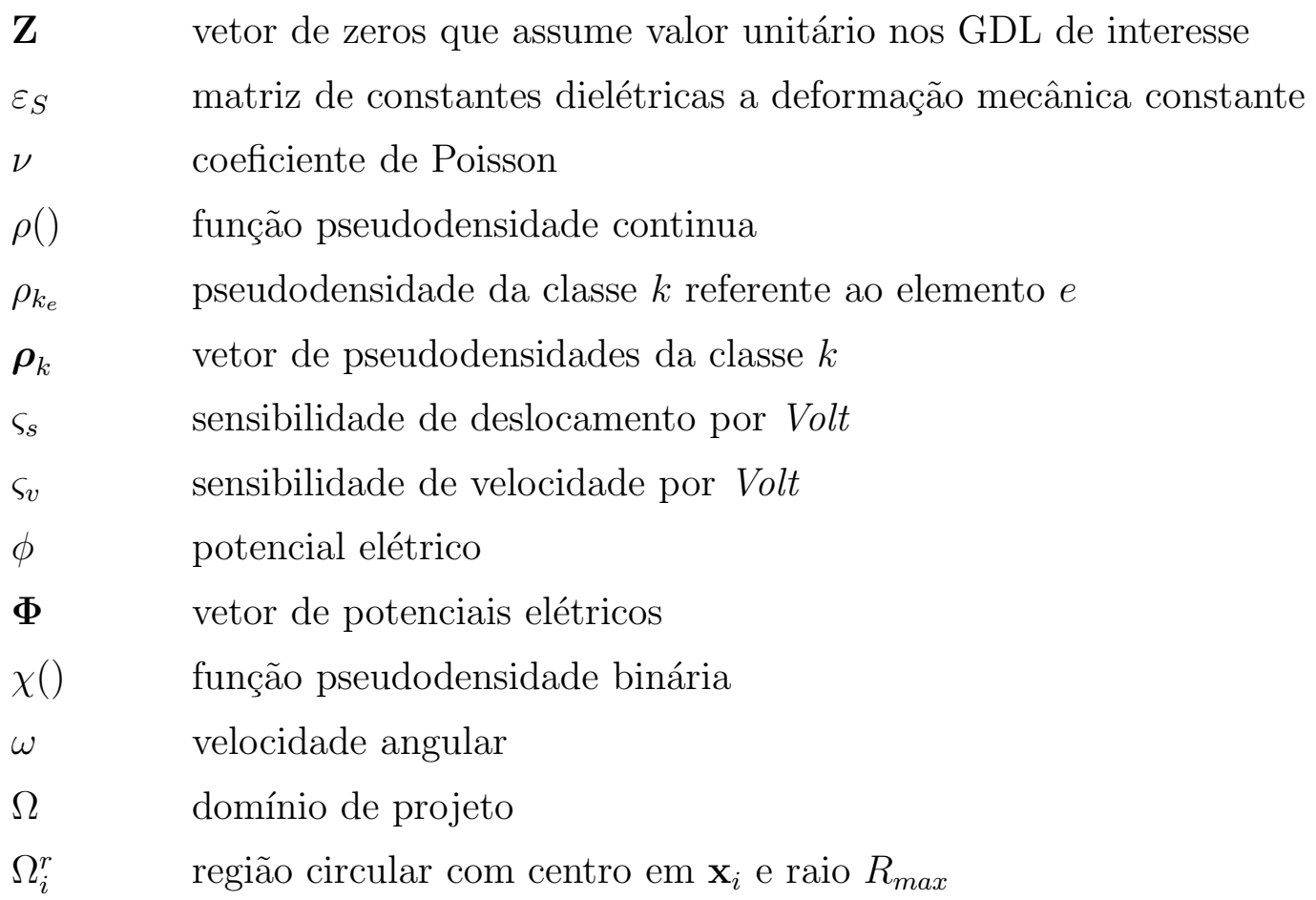




\section{Introdução}

Este trabalho é dedicado ao projeto e à fabricação de dispositivos que utilizam cerâmicas piezelétricas, associadas a estruturas com gradação funcional de material, para fins de atuação e sensoriamento. Para isso, o Método de Otimização Topológica (MOT) é empregado na otimização de suas geometrias e propriedades físicas. A sequência deste Capítulo explora o estado-da-arte dos dispositivos piezelétricos gradados com o intuito de definir os objetivos deste trabalho.

\subsection{Dispositivos Piezelétricos}

Materiais piezelétricos possuem a capacidade de obter energia elétrica quando submetidos a esforços mecânicos e de gerar forças mecânicas internas quando submetidos a um campo elétrico, como é detalhado no Apêndice A. Dentre os materiais com propriedades piezelétricas, os cerâmicos são os mais comumente utilizados em aplicações de engenharia. Porém, as cerâmicas piezelétricas frequentemente não apresentam a conversão entre grandezas elétricas e mecânicas requerida para sua aplicação. Assim, visando estender sua aplicabilidade, é interessante acoplar estruturas flexíveis às cerâmicas. Essas estruturas possuem funcionamento baseado na sua própria flexibilidade estrutural, trabalhando dentro de seu regime elástico e, por isso, sendo utilizados em casos onde deslocamentos de pequena magnitude são esperados. Dessa forma, é possível tanto readequar direção e magnitude dos deslocamentos gerados quanto alterar a intensidade de geração de cargas nos eletrodos das cerâmicas, como é esquematizado na Figura 1.1. Na Figura 1.1(a), um campo elétrico é aplicado à cerâmica piezelétrica de um atuador e, na Figura 1.1(a), uma força mecânica é aplicada a um sensor.

\subsubsection{Aplicações de Dispositivos Piezelétricos}

Os Dispositivos Piezelétricos Flextensionais (DPF), como são denominados, encontram um vasto emprego em atuação e posicionamento de alta precisão, como em microcirurgias (EISINBERG et al., 2001), posicionamento de lentes na interferometria laser (CLAEYSSEN 


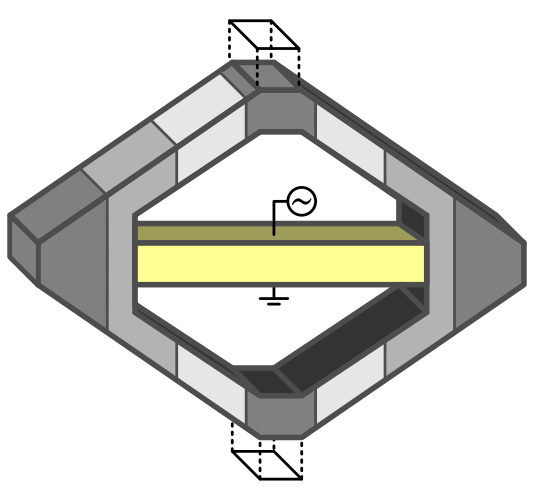

(a)

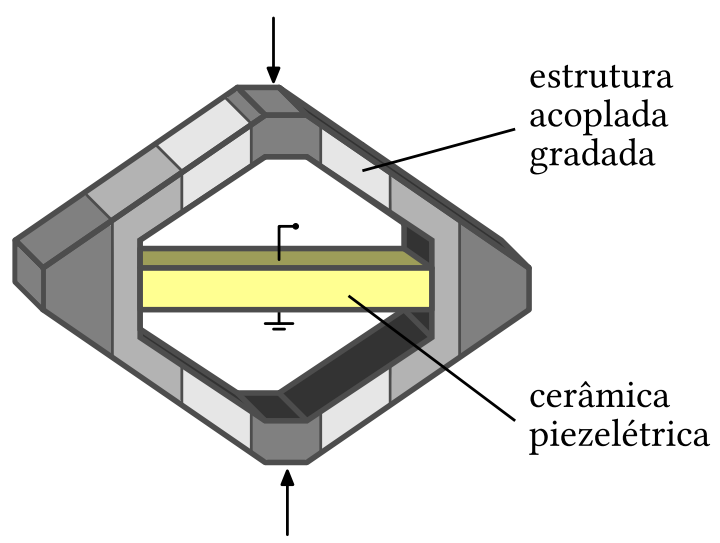

(b)

Figura 1.1: Princípio de funcionamento de (a) atuadores e (b) sensores piezelétricos flextensionais com gradação funcional de material em sua estrutura acoplada.

et al., 2001), micromotores (KWON; CHO; JANG, 2004), Sistemas Microeletromecânicos (MEMS) (REYNAERTS; PEIRS; BRUSSEL, 1998), microscopia eletrônica (SHIBATA et al., 2004), nano-impressão e litografia (CHU; FAN, 2006), máquinas Controle Numérico Computacional (CNC) (WORONKO; HUANG; ALTinTAS, 2003), em agulhas de leitura de discos rígidos (KURIHARA et al., 2004) entre outros. Além disso, também encontram emprego na área de sensoriamento, como em acelerômetros (IULA; LAMBERTI; PAPPALARDO, 1999), giroscópios (TAO et al., 2011) e sonares para tomografia acústica e monitoramento do oceano global (MOROZOV; WEBB, 2003). Há, ainda, aplicações na captação de energia mecânica para conversão em elétrica, pelo Coletor de Energia Piezelétrico (CEP), do inglês energy harvester (RUPP et al., 2009; LIU et al., 2009).

\subsubsection{Projeto de Dispositivos Piezelétricos com Gradação Funcional de Material}

As aplicações de dispositivos piezelétricos podem ser estendidas quando se inclui o conceito de Material com Gradação Funcional (MGF) em seus projetos. Este tipo de material procura reproduzir estruturas naturais cujas propriedades variam gradualmente ao longo de direções específicas. Estudos de algumas estruturas do bioma evidenciaram essa característica em dentes de animais (HUANG et al., 2007) e troncos de bambu (SILVA; WALTERS; PAUlino, 2006). Na Figura 1.2, é possível notar a variação do tamanho das inclusões de um material mais escuro na matriz composta por um material mais claro, constituindo uma gradação funcional suave onde cada material mantém suas características individuais.

Koizumi (1997) relata que a ideia de estruturas artificiais utilizando o conceito de MGF surgiu em meados dos anos 1980. Na época, a motivação principal era obter materiais, para 


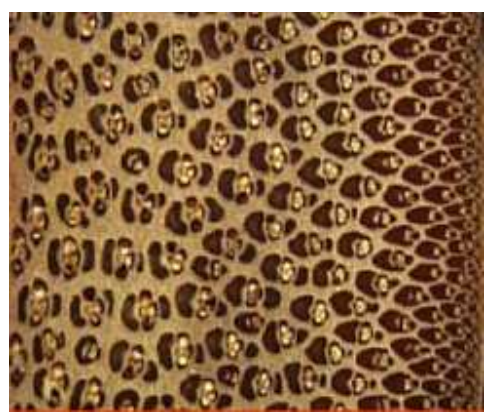

Figura 1.2: Microestrutura da seção transversal do tronco de um bambu (SILVA; WALTERS; PAULINO, 2006).

a indústria aero-espacial, que suportassem gradientes de temperatura superiores a $1600 \mathrm{~K}$. Assim, os MGF cumpriram com essa necessidade, permitindo a fabricação de peças que fossem constituídas por uma face cerâmica, com alta resistência à temperatura, e por uma face metálica, com alta condutibilidade térmica, sem a presença de interfaces concentradoras de tensão entre elas - Figura 1.3.

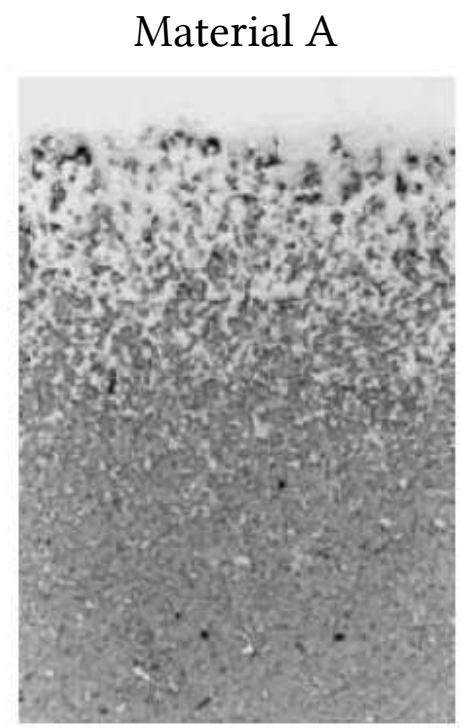

Material B

Figura 1.3: Microestrutura da gradação funcional entre um material A (cerâmico) e um material B (metálico) (KIEBACK; NEUBRAND; RIEDEL, 2003).

Desde Zhu e Meng (1995), os MGF têm sido utilizados com sucesso no projeto de dispositivos piezelétricos, proporcionando ganhos significativos em desempenho e durabilidade. Almajid, Taya e Hudnut (2001), Shin et al. (2001) e Qiu et al. (2003) demonstraram que a resistência à fadiga de atuadores piezelétricos pode ser aprimorada através da utilização de MGF, visto que estes levam a uma distribuição mais suave de tensões na estrutura. Sabe-se, que a utilização de multimateriais em transdutores piezelétricos pode ser vantajosa, como no caso dos atuadores bilaminares projetados por Elka, Elata e Abramovich (2004), e dos atuadores rainbow projetados por Haertling (1994). Já Ichinose, 
Miyamoto e Takahashi (2004) demonstram que a utilização de MGF em transdutores ultrassônicos podem tornar suas propriedades mais interessantes para algumas aplicações.

Finalmente, surgem iniciativas de otimização desses dispositivos, através do MOT. No projeto de atuadores gradados bilaminares (CARBONARI; SILVA; PAULINO, 2006) e multiatuados (CARBONARI et al., 2007; CARBONARI; SILVA; PAULINO, 2009), obtiveram-se resultados maximizando os deslocamentos de saída e minimizando os movimentos acoplados. Já em transdutores ultrassônicos, o emprego do MOT levou a dispositivos gradados que permitem a geração de pulsos mais estreitos e espectros de frequência mais largos do que os convencionais homogêneos (RUBIO et al., 2009). Além disso, Rubio (2010) aplica o MOT no projeto de dispositivos gradados em geral, como ressonadores, transdutores de ultrassom e atuadores flextensionais.

No âmbito mais recente dos atuadores, Carbonari, Silva e Paulino (2010) propõem uma formulação de otimização topológica para o projeto de atuadores piezelétricos integrais, ou seja, que não possuam interfaces entre as cerâmicas e a estrutura acoplada. Dessa forma, evitam-se as imperfeições inerentes à colagem das cerâmicas ao metal, aumentando, por consequência, seu desempenho e sua resistência à fadiga. No que tange à manufatura, Rubio (2010) apresenta protótipos de transdutores ultrassônicos com formato simples, enquanto Amigo e Silva (2011) e Amigo, Vatanabe e Silva (2012) desenvolvem um processo de fabricação de dispositivos piezelétricos gradados com formato complexo, obtendo protótipos de atuadores que são verificados experimentalmente.

\subsubsection{Fabricação de Peças com Gradação Funcional de Material}

Kieback, Neubrand e Riedel (2003) discutem as principais técnicas utilizadas para a obtenção de gradações funcionais de material, que se dividem em dois grandes grupos: metalurgia do pó e processamento por fusão. Este último agrupa técnicas baseadas em fundição centrífuga, fundição por sedimentação, solidificação direcional e infiltração. Já no primeiro grupo, as diferenças entre as técnicas são dadas, essencialmente, na formação dos compactos de pó gradados, que são submetidos à posterior sinterização. Estes compactos podem possuir gradação discreta, constituídos por uma sequência de camadas homogêneas, ou contínua, através da variação gradual da proporção entre os pós ao longo de uma direção. Em vista dos recursos disponíveis no Departamento de Engenharia Mecatrônica e de Sistemas Mecânicos da Escola Politécnica da Universidade de São Paulo, este trabalho se baseia na metalurgia do pó, contando com compactos produzidos pelo empilhamento de camadas compostas por misturas homogêneas dos pós constituintes.

Dentre as técnicas de sinterização disponíveis, a Sinterização por Jato de Plasma (SJP) 
combina características de diferentes processos pela submissão simultânea do material a uma pressão mecânica e a um campo eletromagnético. Esta técnica se destaca em relação às outras devido a sua rapidez e por trabalhar em temperaturas inferiores às dos demais, adequando-se bem à sinterização de materiais com estruturas nanométricas e de MGF.

No equipamento utilizado neste trabalho (SPS-1050), a sinterização é feita pela aplicação de correntes de até $5000 A$ e submissão da amostra a forças de compressão de até $100 k N$ (SPS SYNTEX INC., 2008). Sua alimentação é AC trifásica (200/220V, 50/60Hz e 300A) e gera uma saída em corrente contínua pulsada (até $12 \mathrm{~V}$ e $5000 \mathrm{~A}$ ). O gerador de pulso ON-OFF pode ser configurado de modo que atinja uma frequência máxima de pouco mais de $150 \mathrm{~Hz}$.

O processo de sinterização ocorre dentro de uma câmara feita em aço inoxidável conectada a duas bombas mecânicas que produzem um vácuo de até $6 P a\left(4,5 \times 10^{-2}\right.$ Torr $)$, embora também seja possível trabalhar com gás inerte ou ar atmosférico dependendo da aplicação. A pressão da câmara é medida via manômetros de Bourdon e Pirani. A temperatura de operação pode chegar a $2000^{\circ} \mathrm{C}$ e é aferida via termopar ou pirômetro.

Tipicamente, utilizam-se moldes feitos em grafite condutor que cercam o pó do material sinterizante, conforme é ilustrado na Figura 1.4. Nessa montagem, a corrente elétrica é aplicada diretamente à amostra, proporcionando altas taxas de aquecimento por Efeito Joule.

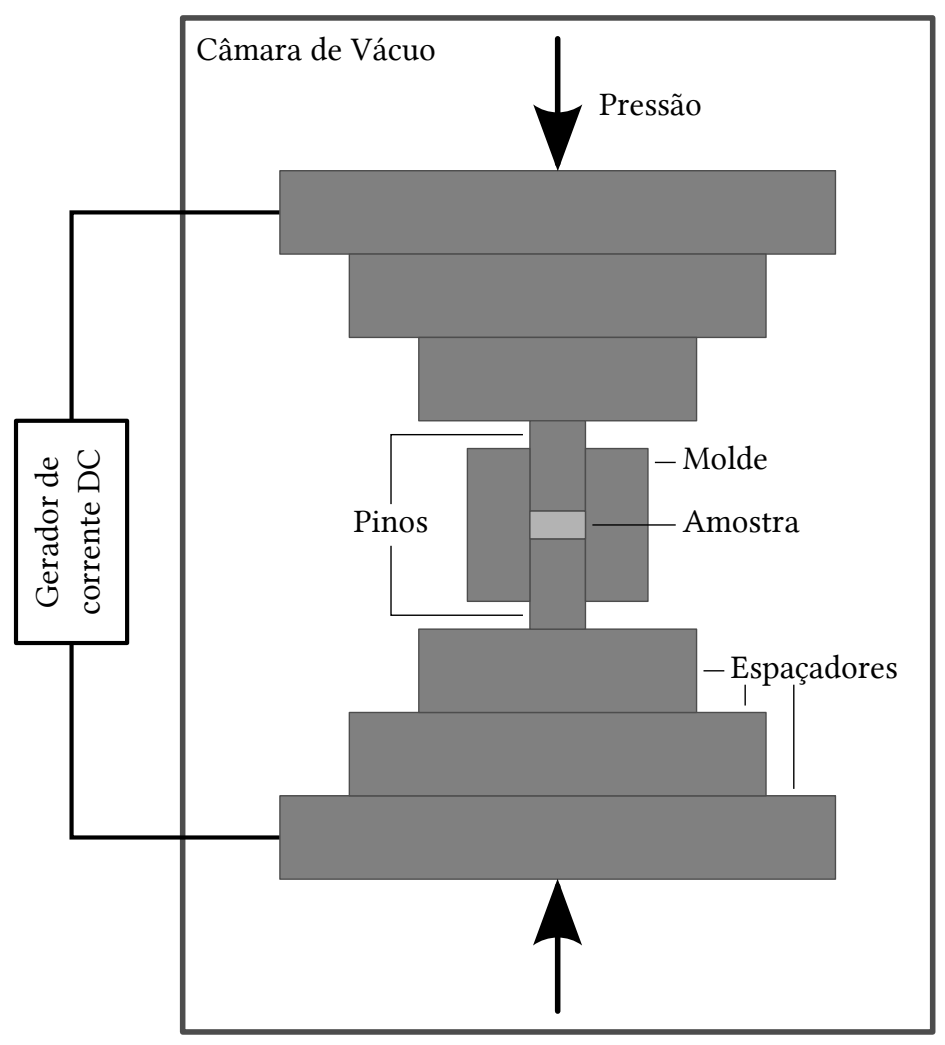

Figura 1.4: Vista em corte da montagem para SJP. 


\subsection{Objetivos}

O objetivo deste trabalho é o estudo da implementação do MOT no projeto de dispositivos piezelétricos que explorem o conceito de MGF. A otimização é focada nas estruturas acopladas às piezocerâmicas, que possuem a função de maximizar os deslocamentos mecânicos de uma região em resposta à excitação por potencial elétrico (Figura 1.1(a)) ou de maximizar a diferença de potencial elétrico entre os eletrodos em resposta à excitação por forças mecânicas (Figura 1.1(b)). Para tanto, são enunciados problemas que consideram simultaneamente a distribuição de material no domínio e a gradação funcional de material no projeto de atuadores e sensores piezelétricos. Por fim, utilizam-se as técnicas de SJP, de Eletro-Erosão a Fio (EEF) e de Corte a Laser (CL) para a fabricação de protótipos, visando verificar experimentalmente os resultados numéricos obtidos.

O procedimento de projeto adotado neste trabalho é esquematizado na Figura 1.5, pelo qual um domínio inicial é discretizado em elementos finitos para a obtenção de topologias e gradações ótimas pela implementação do MOT. Estes resultados são pós-processados e verificados numericamente para a subsequente fabricação. Finalmente, os protótipos fabricados são submetidos a ensaios para a verificação experimental do resultados numéricos.

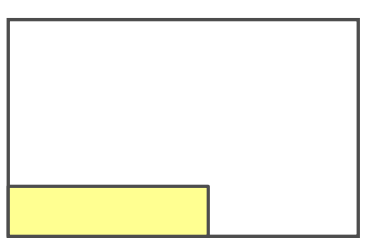

Domínio Inicial

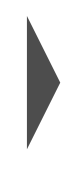

Topologia e Gradação Ótimas Obtidas

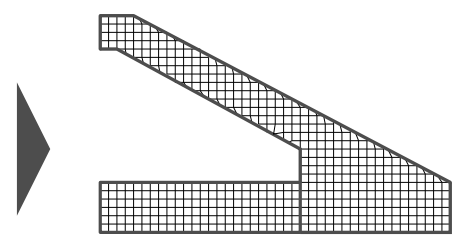

Verificação Numérica
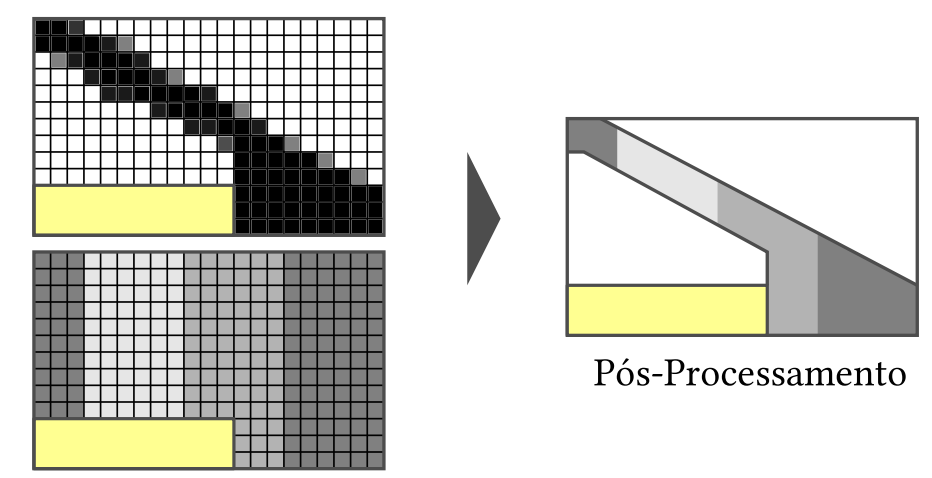

Pós-Processamento

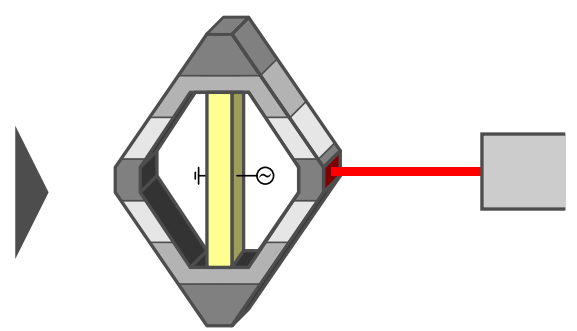

Fabricação

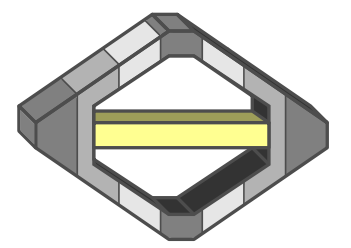

Verificação Experimental

Figura 1.5: Sequência do desenvolvimento de dispositivos piezelétricos com gradação funcional de material. 


\subsection{Justificativa}

Embora métodos sistemáticos, como o MOT, venham sendo empregados no projeto de dispositivos piezelétricos (CARBONARI; SILVA; PAULINO, 2006; CARBONARI et al., 2007; CARBONARI; SILVA; PAULINO, 2009, 2010; RUBIO, 2010), as iniciativas de incorporação do conceito de MGF se focam na gradação das cerâmicas. A utilização de gradações funcionais nas estruturas acopladas de dispositivos flextensionais ainda não foi abordada e, assim, não se sabe ao certo quais vantagens pode proporcionar. Além disso, a fabricação e verificação experimental desses dispositivos relatada na literatura se limita a transdutores ultrassônicos e outros dispositivos de formato simples. Assim, falta verificar a viabilidade da fabricação de peças gradadas metálicas com formato complexo a partir de tarugos MGF.

\subsection{Organização do Texto}

Este trabalho se inicia com a apresentação dos diferentes métodos de otimização estrutural no Capítulo 2, dando ênfase ao Método de Otimização Topológica (MOT), com a exposição de alguns conceitos e aspectos numéricos inerentes à implementação deste para estruturas em MGF. Na sequência do mesmo Capítulo, é desenvolvida a formulação teórica da otimização de dispositivos piezelétricos, especificando o Modelo de Material (MM) utilizado e as funções-objetivo dos problemas. De posse dessas formulações, descreve-se a implementação numérica da otimização no Capítulo 3, valendo-se do MEF e de métodos matemáticos de programação como a Programação Linear Sequencial (PLS). Dessa implementação, são obtidas topologias ótimas para os problemas expostos no Capítulo 5. Porém, devido à necessidade de validação destes, propõe-se um processo de fabricação de dispositivos piezelétricos gradados no Capítulo 4, que são a base dos resultados experimentais apresentados no Capítulo 6. Finalmente, as conclusões do trabalho são apresentadas no Capítulo 7. Ao longo do texto, determinados assuntos são tratados em maior profundidade em Apêndices, que abordam os temas de piezeletricidade, MEF e PLS. Além disso, ainda são incluídos Anexos, que apresentam relações entre constantes elásticas e propriedades de material utilizados. 


\section{Projeto de Dispositivos Piezelétricos Gradados}

Para o projeto de dispositivos piezelétricos com distribuição ótima de material, implementa-se o MOT baseado em funções-objetivo que avaliam a transdução eletromecânica. Neste Capítulo, são desenvolvidas formulações que consideram a otimização da topologia e da lei de gradação das estruturas acopladas desses dispositivos, através de duas classes de variáveis de projeto.

\subsection{Métodos de Otimização Estrutural}

Em projetos de engenharia, a experiência acumulada ao longo dos anos costuma definir as características de um produto. No entanto, existem formas sistemáticas para obtenção de estruturas que otimizem algum objetivo estabelecido. Essas formas podem ser classificadas sob três métodos numéricos de otimização principais: paramétrica, de forma e topológica Figura 2.1.

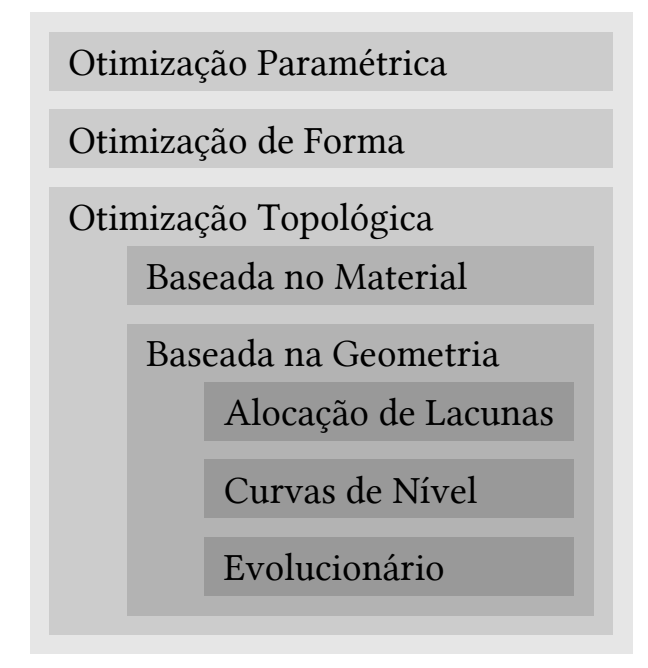

Figura 2.1: Classificação dos métodos de otimização estrutural.

No Método de Otimização Paramétrica (MOP) (VANDERPlaATS, 1984), o formato da estrutura é definido previamente e as variáveis de projeto são dimensões ou proporções. Na 
viga do exemplo da Figura 2.2(a), as variáveis são as áreas transversais das barras da treliça.

No Método de Otimização de Forma (MOF) (HAFTKA; GURDAL; KAMAT, 1990), as variáveis de projeto são parâmetros de geometrias primitivas que definem os contornos da estrutura. No exemplo da Figura 2.2(b), as variáveis são as coordenadas dos pontos de controle das splines que dão forma aos vazios na viga.

Finalmente, no MOT (BENDSOE; KIKUCHI, 1988) as variáveis de projeto são propriedades de cada porção de uma malha definida sobre um Domínio Fixo Estendido (DFE). Desse modo, ao projetista cabe apenas definir as condições de contorno e as cargas aplicadas à estrutura, já que o próprio método se encarregará de criar regiões vazias pela variação dessas propriedades. No exemplo da Figura 2.2(c), a propriedade variada é a pseudodensidade de cada elemento da malha, alterando proporcionalmente sua rigidez e representando, dessa forma, presença ou ausência de material. O MOT é, portanto, o mais geral dos métodos aqui tratados, sendo o que menos limita o espaço de soluções do problema.

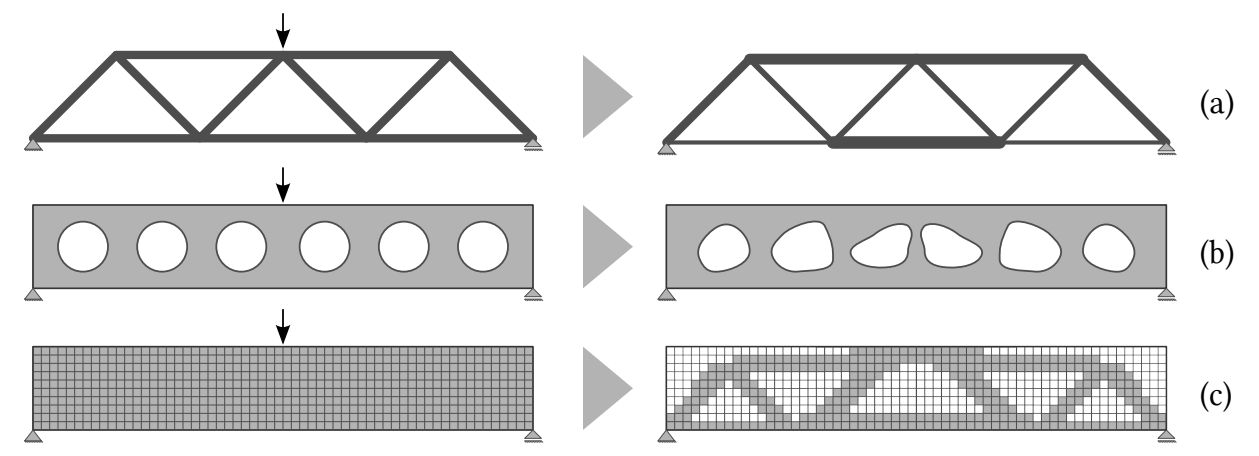

Figura 2.2: Exemplo de aplicação dos métodos de otimização (a) paramétrica, (b) de forma e (c) topológica.

O campo da otimização topológica pode ainda ser subdividido em duas abordagens: a baseada na geometria e a baseada no material. A abordagem baseada na geometria possui três métodos principais: de alocação de lacunas, de curvas de nível e evolucionários. O Método de Otimização Topológica por Alocação de Lacunas (MOTAL), ou bubble method (NOVOTNY et al., 2003), altera a estrutura através da distribuição de lacunas pelo domínio. O Método de Otimização Topológica por Curvas de Nível (MOTCN), ou level set method (SETHIAN; WIEGMANN, 2000), calcula uma superfície cuja curva de nível de determinada cota representará o contorno da estrutura. O Método de Otimização Topológica Evolucionária (MOTE) utiliza critérios heurísticos para alocar e remover elementos da malha (CHU et al., 1996). Já a abordagem baseada no material considera a existência de uma microestrutura que combine materiais distintos em cada célula unitária do domínio (método de homogeneização (BENDSOE; KIKUCHI, 1988)) ou a existência de materiais intermediários (método de pseudodensidade (BENDSOE, 1989)) - ver Seção 2.2.1. 
As primeiras iniciativas de otimização de dispositivos piezelétricos foram baseadas em modelos analíticos, como no caso do projeto de transdutores apresentado por Kossoff (1966). Porém, esses modelos são válidos apenas para casos particulares ou introduzem simplificações que o distanciam demasiadamente dos casos reais. Na década de 70, trabalhos como o de Allik e Hughes (1970) tornaram o projeto desses dispositivos mais sistemáticos, introduzindo o MOP e o MOF. Finalmente, trabalhos como Silva, Nishiwaki e Kikuchi (2000), Carbonari, Silva e Paulino (2006), Carbonari et al. (2007), Carbonari, Silva e Paulino (2009), Rubio et al. (2009), Rubio (2010) e Carbonari, Silva e Paulino (2010) utilizaram o MOT, baseado em pseudodensidades, no projeto de atuadores e sensores piezelétricos, comprovando a robustez deste método nesta aplicação. Portanto, neste trabalho será seguida a mesma abordagem destes últimos autores.

\subsection{Método de Otimização Topológica}

\subsubsection{Modelo de Material}

O material de uma célula unitária do DFE, com centroide em $\mathbf{x}$, pode ser definido por uma função pseudodensidade $\chi(\mathbf{x})$.

$$
\chi(\mathbf{x})= \begin{cases}0, & \text { material } \alpha \\ 1, & \text { material } \beta\end{cases}
$$

Porém, a restrição da solução ao espaço binário levaria à formulação de um problema mal-posto, pois apresenta múltiplos mínimos locais (SIGMUND; PETERSSON, 1998). Com isso, é necessário relaxar o problema, supondo uma mistura de materiais em cada célula. A forma de representação dessa mistura é denominada Modelo de Material (MM), e se divide em modelos naturais e artificiais.

Nos modelos naturais, considera-se uma microestrutura compósita, cuja geometria é parametrizável. As propriedades efetivas do material compósito podem, então, ser obtidas por um método de homogeneização (BENDSOE; KIKUCHI, 1988).

Já nos modelos artificiais, que este trabalho utiliza, uma função contínua $\rho(\mathbf{x})$, que assume valores entre zero e um, é utilizada para ponderar as propriedades $\mathcal{P}(\mathbf{x})$ dos materiais $\alpha$ e $\beta, \mathcal{P}_{\alpha}$ e $\mathcal{P}_{\beta}$ afim de obter as propriedades da célula unitária. Assim:

$$
\mathcal{P}(\mathbf{x})=[1-\rho(\mathbf{x})] \mathcal{P}_{\alpha}+\rho(\mathbf{x}) \mathcal{P}_{\beta}
$$




\subsubsection{Escala de Cinza}

A relaxação do problema para a formulação contínua, introduzida na Seção anterior, faz com que a estrutura obtida pelo MOT apresente a chamada escala de cinza (Figura 2.3), que deixa indefinido material daquela região. Assim, neste caso, é desejável que a função $\rho(\mathbf{x})$ da Eq.(2.2) assuma valores extremos - zero ou um. Para minimizar a ocorrência de valores intermediários, coeficientes penalizadores podem ser incluídos na formulação, como no caso do modelo Material Isotrópico Sólido com Penalização (MISP) proposto por Bendsoe (1989):

$$
\mathcal{P}(\mathbf{x})=\left[1-\rho^{p}(\mathbf{x})\right] \mathcal{P}_{\alpha}+\rho^{p}(\mathbf{x}) \mathcal{P}_{\beta}
$$

onde o coeficiente penalizador $p \geq 1$.

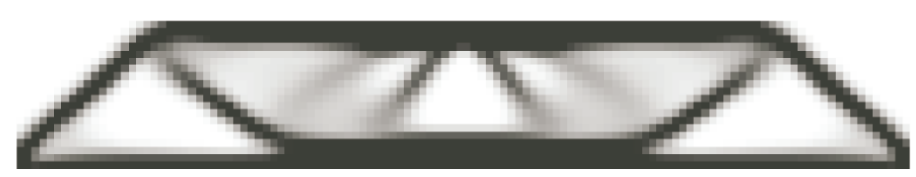

Figura 2.3: Ocorrência de escala de cinza (KIYONO, 2008).

Porém, conforme se aumenta a penalização, mais mínimos locais são criados. Afim de contornar esse problema, Svanberg (1994) propôs o Método da Continuação (MC), no qual o valor do coeficiente $p$ é inicialmente unitário e, no decorrer da iterações do MOT, é gradualmente incrementado. Dessa forma, diminui-se a possibilidade da solução recair sobre ótimos locais muito distantes do ótimo global, conforme ilustrado na Figura 2.4.

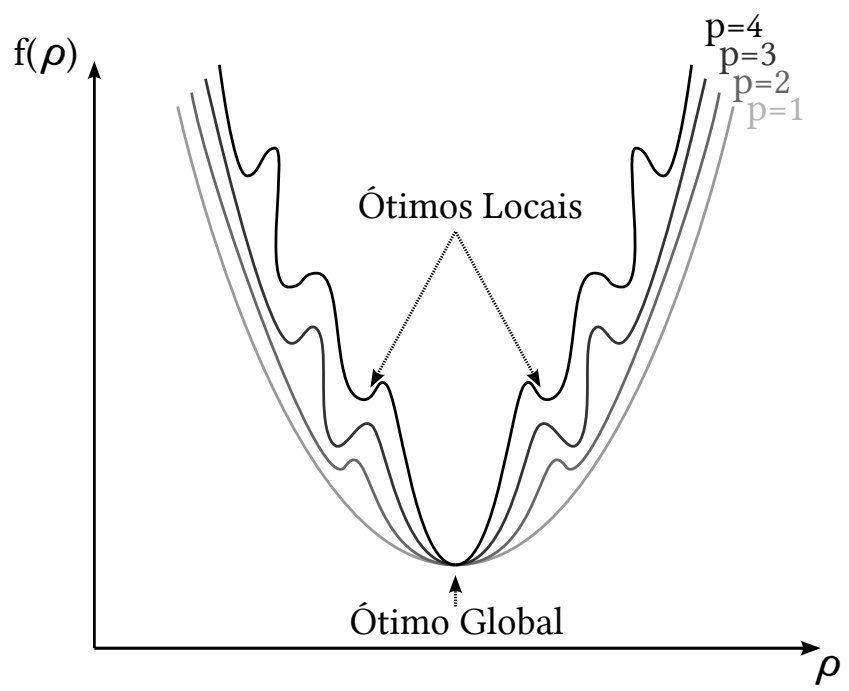

Figura 2.4: Demonstração do aumento de mínimos locais provocado pelo aumento dos penalizadores.

Porém, para as variáveis de gradação não se introduz penalizadores, já que, neste caso, materiais intermediários são manufaturáveis. 


\subsubsection{Instabilidade de Tabuleiro e Dependência de Malha}

Na implementação numérico do MOT, as topologias obtidas podem apresentar regiões onde elementos de materiais diferentes se alternam, demarcando o quadriculado da malha conforme destacado na Figura 2.5. A ocorrência desse tipo de arranjo é denominada instabilidade de tabuleiro e, evidentemente, é indesejada pois não corresponde a uma topologia manufaturável.

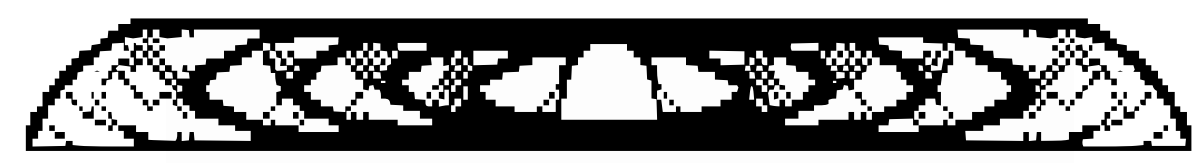

Figura 2.5: Ocorrência da instabilidade de tabuleiro (KIYONO, 2008).

Kikuchi, Hollister e Yoo (1997) investigaram esse fenômeno e constataram que sua ocorrência se deve às aproximações numéricas introduzidas pelo MEF, que fazem com que este arranjo seja mais rígido ao cisalhamento do que o mesmo volume de material disposto continuamente. Além disso, esta instabilidade também se deve ao fato do MOT ser formulado através de um problema variacional misto, ou seja, com o objetivo de determinar dois campos físicos: o campo de deslocamentos e o campo de densidades. De fato, Jog e Haber (1996) demonstram que o sistema de equações do MOT se torna mal-condicionado para alguns campos de deslocamentos quando se utilizam elementos finitos de baixa ordem no MEF.

Outro fenômeno inerente à implementação numérica do MOT é a influência do grau de discretização da malha do MEF na topologia resultante. Esta dependência de malha é a manifestação numérica da ausência de solução do problema, que busca estruturas com membros mais delgados conforme maior é o número de elementos no DFE. A Figura 2.6 mostra a solução de um mesmo problema para diferentes discretizações de malha.

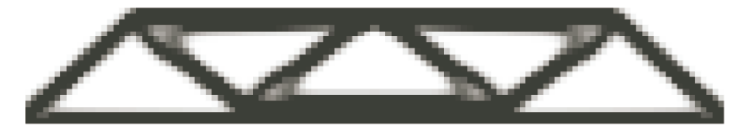

(a)

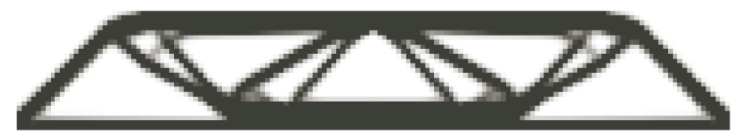

(b)

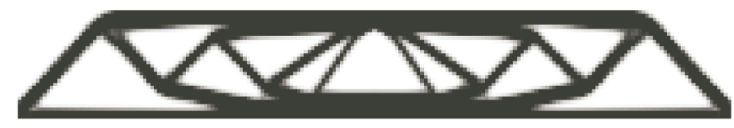

(c)

Figura 2.6: Topologia ótima obtida com malhas de (a) 1200 elementos, (b) 1875 elementos e (c) 3000 elementos (KIYONO, 2008). 
Uma alternativa para mitigar tanto a instabilidade de tabuleiro quanto a dependência de malha é a utilização de filtros espaciais, que regularizam o campo de pseudodensidades pela sua convolução com uma função suave (BOURDIN, 2001). Bruns e Tortorelli (2001) implementaram uma técnica de projeção onde cada pseudodensidade nodal depende das variáveis de projeto associadas aos nós que estejam compreendidos por um raio de abrangência $R_{\max }$ - Figura 2.7. A influência de cada variável de projeto $d_{j}$ é ponderada pela sua distância ao nó $i$ avaliado $\left(R_{i j}\right)$, na forma da Equação (2.5).

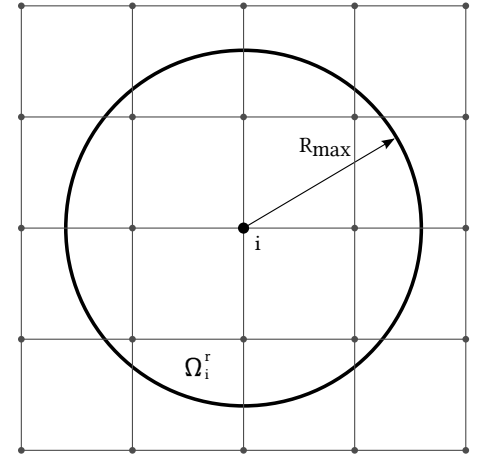

(a)

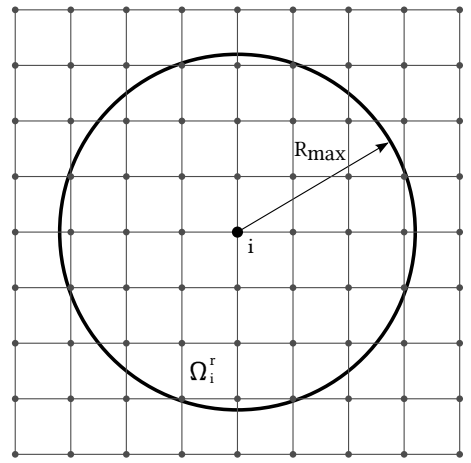

(b)

Figura 2.7: Raio de abrangência do filtro de projeção em (a) malha grossa e (b) malha fina.

$$
R_{i j}=\left\|\mathbf{x}_{j}-\mathbf{x}_{i}\right\|
$$

onde $\mathbf{x}_{i}$ e $\mathbf{x}_{j}$ denotam os vetores de coordenadas dos nós $i$ e $j$.

$$
w_{i j}=\left\{\begin{array}{lr}
\left(\frac{R_{\max }-R_{i j}}{R_{\max }}\right)^{g} & \text { se } \mathbf{x}_{j} \in \Omega_{i}^{r} \\
0 & \text { se } \mathbf{x}_{j} \notin \Omega_{i}^{r}
\end{array}\right.
$$

onde $\Omega_{i}^{r}$ representa a região circular com centro em $\mathbf{x}_{i}$ e raio $R_{\text {max }}$ e o expoente $g$ define a ordem da função peso (NAKASONE; SILVA, 2010), como ilustra a Figura 2.8. Este expoente é utilizado para evitar a ocorrência de materiais intermediários, visto que a influência de nós vizinhos diminui exponencialmente com a distância.

Assim, cada pseudodensidade $\rho_{i}$ será influenciada pelas variáveis de projeto $d_{j}$ ao seu entorno, na forma da Eq. (2.6).

$$
\rho_{i}=\frac{\sum_{j \in S_{i}^{r}} d_{j} w_{i j}}{\sum_{j \in S_{i}^{r}} w_{i j}}
$$

sendo $S_{i}^{r}$ o conjunto dos pontos compreendidos pela região $\Omega_{i}^{r}$. 


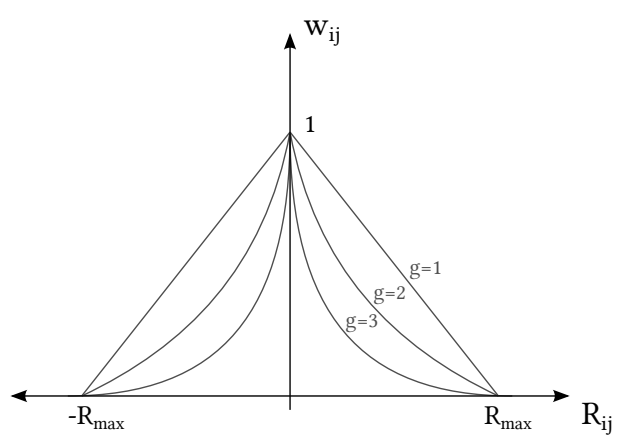

Figura 2.8: Perfis da função peso de acordo com o valor de $g$.

\subsection{Projeto de Dispositivos com Gradação Funcional de Material}

Tendo em vista os conceitos do MOT definidos na Seção anterior, o projeto de dispositivos piezelétricos gradados segue o esquematizado na Figura 1.5, percorrendo as etapas de discretização do domínio, otimização de topologia e gradação, pós-processamento e verificação. Para a etapa de otimização, o Material Isotrópico Sólido com Penalização (MISP), introduzido na Seção anterior, é utilizado para definir as propriedades elásticas do material através da seguinte relação:

$$
\mathbf{c}(\mathbf{x})=\left[1-\rho^{p}(\mathbf{x})\right] \mathbf{c}_{\alpha}+\rho^{p}(\mathbf{x}) \mathbf{c}_{\beta}
$$

onde $\mathbf{c}_{\alpha}$ e $\mathbf{c}_{\beta}$ denotam as propriedades constitutivas elásticas dos materiais isotrópicos $\alpha$ e $\beta$. Sendo que, tipicamente, as propriedades do vazio são atribuídas a um desses materiais.

O mesmo princípio pode ser estendido para a consideração de três materiais (SIGMUND, 2000), $\alpha, \beta$ e $\gamma$, incluindo na formulação duas classes de pseudodensidades, $\rho_{1}$ e $\rho_{2}$, e seus respectivos coeficientes penalizadores, $p$ e $q$ :

$$
\mathbf{c}(\mathbf{x})=\left[1-\rho_{1}^{p}(\mathbf{x})\right] \mathbf{c}_{\alpha}+\rho_{1}^{p}(\mathbf{x})\left(\left[1-\rho_{2}^{q}(\mathbf{x})\right] \mathbf{c}_{\beta}+\rho_{2}^{q}(\mathbf{x}) \mathbf{c}_{\gamma}\right)
$$

Atribuindo-se as propriedades elásticas do vazio a $\alpha$, de um material $A$ a $\beta$ e de um material $B$ a $\gamma$, a expressão do MISP torna-se:

$$
\mathbf{c}(\mathbf{x})=\left[1-\rho_{1}^{p}(\mathbf{x})\right] \mathbf{c}_{\text {vazio }}+\rho_{1}^{p}(\mathbf{x})\left(\left[1-\rho_{2}^{q}(\mathbf{x})\right] \mathbf{c}_{A}+\rho_{2}^{q}(\mathbf{x}) \mathbf{c}_{B}\right)
$$

Como no caso dos MGF não é necessário que os valores de $\rho_{2}$ sejam extremos, já que valores intermediários de rigidez podem ser obtidos pela mistura dos materiais, o coeficiente penalizador $q$ geralmente assume valor unitário. 


\subsubsection{Projeto de Atuadores Piezelétricos}

No projeto de atuadores, o objetivo usual é a maximização do deslocamento estático $\mathbf{u}_{1}$ produzido nas regiões $\Gamma_{t}$ pela aplicação de um potencial elétrico $\phi_{1}$ no eletrodo $\Gamma_{\phi}$. Porém, a topologia resultante do problema de otimização enunciado desta maneira possui rigidez próxima de zero, ou seja, é uma estrutura vazia ou desconexa. Portanto, para garantir uma rigidez estrutural mínima, molas de constante elástica $k_{m}$ são dispostas nas faces de atuação.

O domínio de projeto para a maximização do deslocamento de saída é ilustrado na Figura 2.9 .

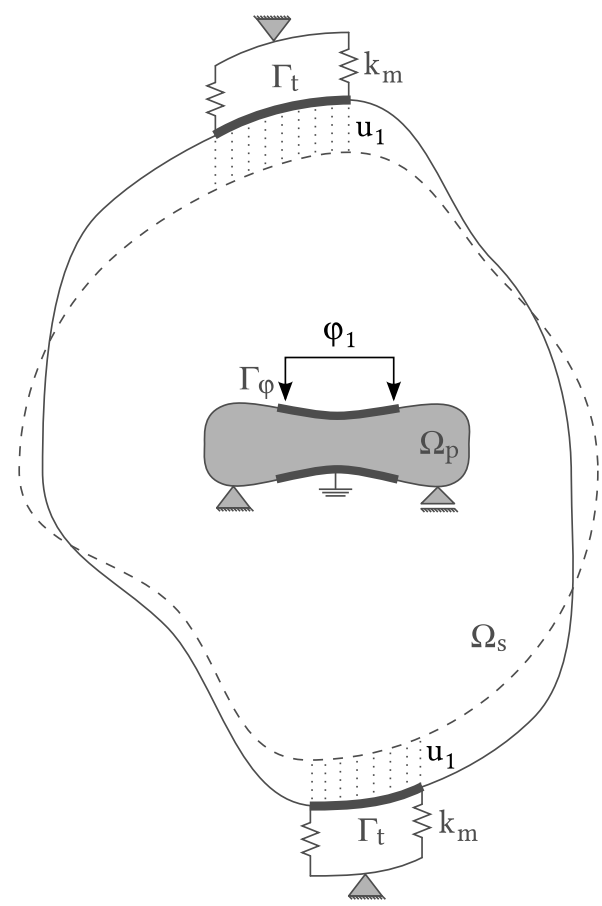

Figura 2.9: Caso de carga para o cálculo do deslocamento estático gerado.

Nos atuadores piezelétricos projetados por este trabalho, considera-se um domínio piezelétrico fixo $\Omega_{p}$ e otimiza-se apenas a estrutura acoplada contida no domínio $\Omega_{s}$. Assim, a formulação contínua da função-objetivo de maximização do deslocamento estático gerado é dada por:

$$
\begin{aligned}
& \text { Maximizar : } \mathcal{F}_{a}=L_{1}=\int_{\Gamma_{t}} \mathbf{u}_{1}\left(\rho_{1}, \rho_{2}\right) d \Gamma \\
& \rho_{1}(\mathbf{x}), \rho_{2}(\mathbf{x}) \\
& \text { tal que : } 0 \leq \rho_{1}, \rho_{2} \leq 1 \\
& \int_{\Omega_{s}} \rho_{1} d \Omega_{s}-\Theta_{1} \leq 0 \\
& \int_{\Omega_{s}}^{\Omega_{s}} \rho_{1} \rho_{2} d \Omega_{s}-\Theta_{2} \int_{\Omega_{s}} \rho_{1} d \Omega_{s} \leq 0 \\
& \text { Equilíbrio Piezelétrico - Eq. (B.14) }
\end{aligned}
$$


onde $\mathbf{u}_{1}$ denota os deslocamentos gerados ao longo do domínio pela aplicação do potencial elétrico, $\Omega_{s}$ é o domínio otimizável, $\Theta_{1}$ é o valor máximo desejado para o volume final e $\Theta_{2}$ limita a proporção de cada material da gradação na estrutura. A necessidade dessas restrições é discutida mais adiante, nas Seções 5.1 e 5.2.

\subsubsection{Projeto de Sensores Piezelétricos}

Os mesmos conceitos definidos anteriormente encontram aplicação no projeto de sensores piezelétricos, onde se estimam carregamentos mecânicos pela diferença de potencial que estes produzem entre os eletrodos de uma cerâmica piezelétrica. Assim, a sensibilidade de um sensor é proporcional à tensão elétrica de saída $\phi_{1}$ gerada no eletrodo $\Gamma_{\phi}$ por uma excitação mecânica $\mathbf{f}_{1}$ na face de sensoriamento $\Gamma_{t}$. Porém, a topologia resultante deste problema de otimização possui rigidez próxima de zero, ou seja, é uma estrutura vazia ou desconexa. Portanto, para garantir uma rigidez estrutural mínima, molas de constante elástica $k_{m}$ são dispostas nas faces de sensoriamento, como artifício para evitar deslocamentos artificialmente grandes.

O domínio de projeto para a maximização da sensibilidade de um sensor é ilustrado na Figura 2.10.

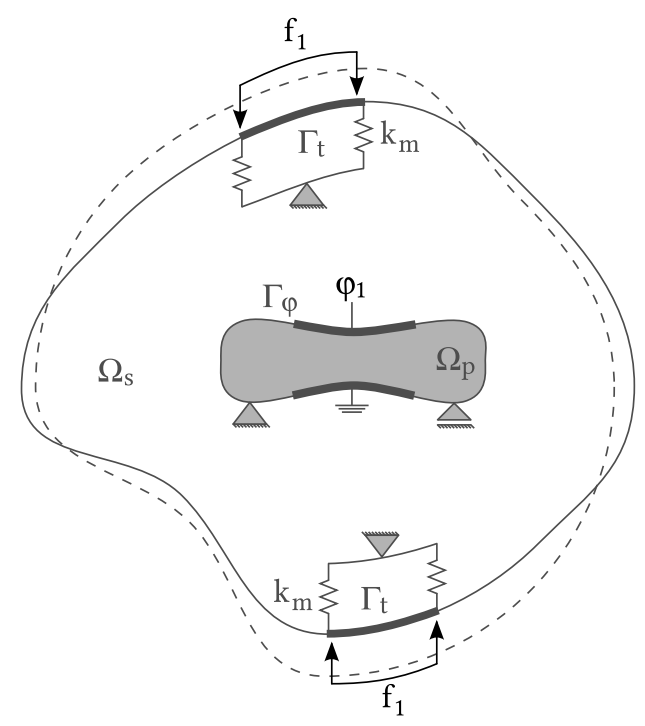

Figura 2.10: Caso de carga para o cálculo do potencial elétrico estático gerado.

Nos sensores piezelétricos projetados por este trabalho, considera-se um domínio piezelétrico fixo $\Omega_{p}$ e otimiza-se apenas a estrutura acoplada contida no domínio $\Omega_{s}$. Assim, 
o problema de otimização para o projeto de sensores piezelétricos é, então, dado por:

$$
\begin{aligned}
& \text { Maximizar : } \mathcal{F}_{s}=L_{1}=\int_{\Gamma_{\phi}} \phi_{1}\left(\rho_{1}, \rho_{2}\right) d \Gamma \\
& \rho_{1}(\mathbf{x}), \rho_{2}(\mathbf{x}) \\
& \text { tal que : } 0 \leq \rho_{1}, \rho_{2} \leq 1 \\
& \int_{\Omega_{s}} \rho_{1} d \Omega_{s}-\Theta_{1} \leq 0 \\
& \int_{\Omega_{s}}^{\Omega_{s}} \rho_{1} \rho_{2} d \Omega_{s}-\Theta_{2} \int_{\Omega_{s}} \rho_{1} d \Omega_{s} \leq 0
\end{aligned}
$$

Equilíbrio Piezelétrico - Eq. (B.14)

onde $\Omega_{s}$ é o domínio otimizável, $\Theta_{1}$ é o valor máximo desejado para o volume final e $\Theta_{2}$ define a proporção de cada material da gradação na estrutura. A necessidade dessas restrições é discutida mais adiante, na Seção 5.3. 


\section{Implementação Numérica}

Em problemas de otimização topológica, são esperadas geometrias complexas descritas por um número muito grande de variáveis. Logo, é preciso adequar as formulações contínuas do Capítulo 2 para a utilização do MEF e de métodos matemáticos de programação. Neste Capítulo, discute-se como é feita essa adequação e em quais instabilidades numéricas ela acarreta.

\subsection{Aspectos Numéricos da Implementação do MOT}

\subsubsection{Aproximação Contínua da Distribuição de Material}

Da mesma forma que o campo de deslocamentos é interpolado no interior de um elemento finito, na otimização de estruturas MGF é interessante parametrizar também o campo de pseudodensidades. Isto porque são esperadas transições graduais de um material a outro.

Neste trabalho é utilizada a Aproximação Contínua da Distribuição de Material (ACDM) - Matsui e Terada (2004). Nessa abordagem, a propriedade otimizável de um elemento é dada pela interpolação das propriedades avaliadas em cada um de seus nós, ou seja:

$$
\rho(\mathbf{x})=\sum_{I=1}^{n_{d}} \rho_{I} N_{I}(\mathbf{x})
$$

onde $n_{d}$ é o número de nós do elemento finito definido em $\mathbf{x}, \rho_{I}$ denota a pseudodensidade nodal e $N_{I}$ são as funções de forma detalhadas no Apêndice B.

\subsubsection{Linearização das Funções-Objetivo e das Restrições}

Por envolver uma quantidade vultosa de variáveis, os problemas de otimização topológica costumam ser resolvidos por métodos de programação matemática como o Método das Assíntotas Móveis (MMA) (SVANBERG, 1987) e a Programação Linear Sequencial (PLS), que é o método adotado neste trabalho e é detalhado no Apêndice C). Em ambos os métodos, há necessidade de linearização das funções do problema, que é feita baseada nas séries de 
Taylor, como mostrado na Equação (3.2) para uma função $f$ genérica:

$$
f\left(d_{j}\right)=\sum_{z=0}^{\infty}\left[\frac{\partial^{(z)} f}{\partial d_{j}^{(z)}}\right]_{d_{j}=d_{j}^{0}} \frac{\left(d_{j}-d_{j}^{0}\right)^{z}}{z !}
$$

onde $d_{j}$ denota a variável de projeto $j$ dada pela Equação $(2.6)$, com $d_{j}^{0}$ representando o ponto em torno do qual a aproximação é válida. Para que essa aproximação polinomial se torne linear, basta desprezar os termos onde $z>1$.

Dessa forma, para a implementação do problema de otimização, será preciso obter os gradientes da função-objetivo e respectivas funções restritivas, ou seja, calcular as sensibilidades do problema.

As sensibilidades podem ser obtidas analiticamente ou por aproximações numéricas (HAFTKA; GURDAL; KAMAT, 1990). Quando aproximadas numericamente, aplica-se o Método de Diferenças Finitas (MDF), que é pouco eficiente computacionalmente e tem precisão condicionada à escolha adequada do passo. Já quando obtidas analiticamente, embora exijam um desenvolvimento matemático mais elaborado, o valor resultante é exato e o custo computacional é relativamente baixo.

Este trabalho calcula analiticamente as sensibilidades das funções envolvidas no problema de otimização em relação às variáveis de projeto do vetor $\mathbf{d}_{k}$, onde $k \in\{1 ; 2\}$ designa a classe (1 para topologia ou 2 para gradação) das variáveis destes vetores (ver Seção 2.2.1). Como as funções-objetivo não dependem diretamente das variáveis de projeto, mas sim das pseudodensidades, é preciso realizar a mudança de variável, que é descrita na Seção 3.3.2.

\subsection{Procedimento de Otimização}

Este trabalho utiliza um software desenvolvido em linguagem MATLAB, cujo algoritmo é descrito pelo fluxograma da Figura 3.1. Este software inclui a implementação do MEF, que é verificada no Apêndice B.6.

Os dados fornecidos pelo usuário abrangem características do DFE (dimensões, materiais admissíveis em cada região, condições de contorno etc.) e parâmetros do MOT (valores iniciais de pseudodensidade, valor inicial e variação dos coeficientes de penalização etc.). Considera-se que a solução convergiu quando a variação do valor da função-multiobjetivo entre duas iterações consecutivas é menor do que o valor tolerável. 


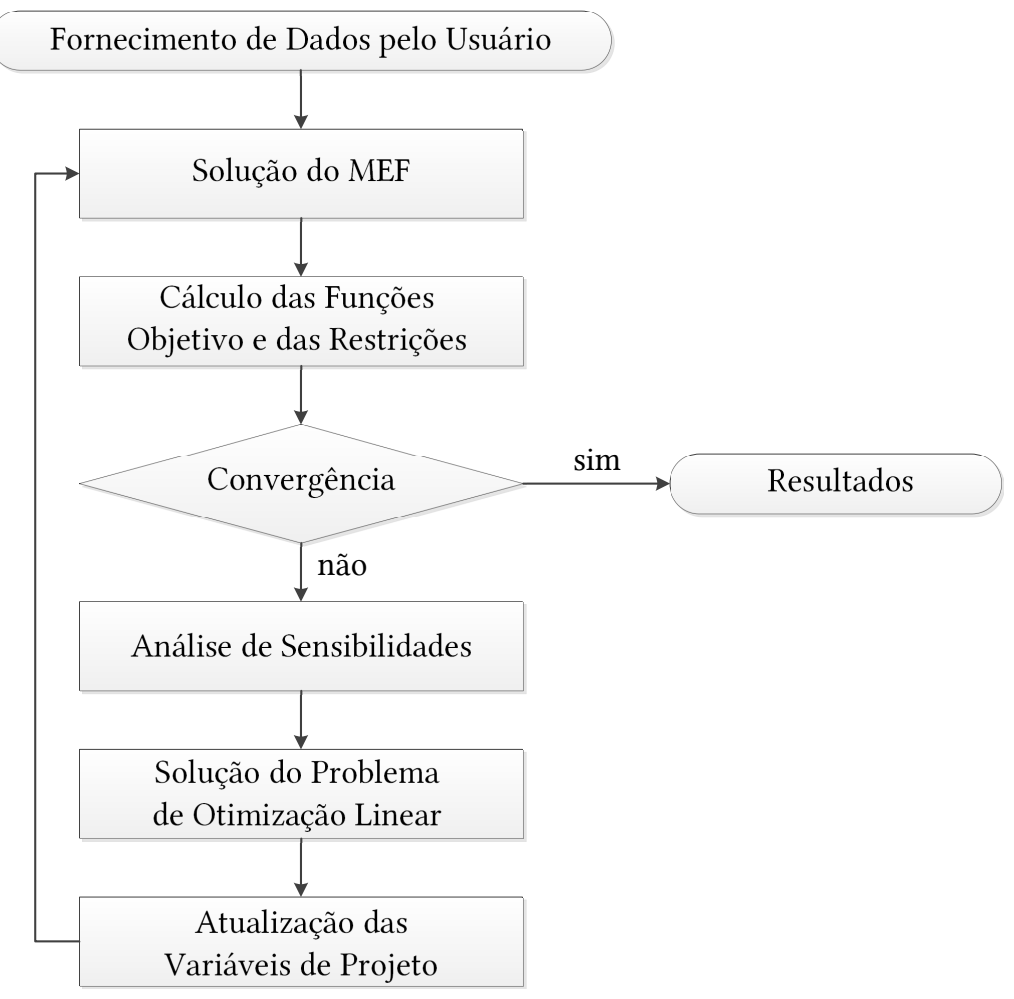

Figura 3.1: Fluxograma do software de implementação do MOT.

\subsection{Otimização de Atuadores Piezelétricos}

Para a implementação, via MEF, do problema de otimização formulado na Seção 2.3.1, é necessário reescrevê-lo em termos de graus de liberdade dos elementos finitos do domínio de projeto, como descrito na Seção 3.3.1. Além disso, visto que o PLS se baseia nas séries de Taylor de primeira ordem, também é preciso obter as sensibilidades das funções-objetivo, como é demonstrado na Seção 3.3.2.

\subsubsection{Formulação Discreta}

A função-objetivo para otimização de atuadores piezelétricos, dada pela Equação (2.10), pode ser reescrita no domínio discreto da seguinte forma:

$$
L_{1}=\mathbf{Z}_{\Gamma_{t}}^{t} \mathbf{U}_{1}
$$

onde $\mathbf{U}_{1}$ é o vetor de deslocamentos mecânicos e $\mathbf{Z}_{\Gamma_{t}}$ denota um vetor de zeros que assume valor unitário nos graus de liberdade referentes ao deslocamento de saída desejado em $\Gamma_{t}$.

Portanto, para a implementação do MEF, o problema de otimização de atuadores piezelétricos se torna: 


$$
\begin{aligned}
\text { Maximizar : } & \mathcal{F}_{a}=L_{1}=\mathbf{Z}_{\Gamma_{t}}^{t} \mathbf{U}_{1} \\
\mathbf{d}_{1}, \mathbf{d}_{2} & \\
\text { tal que : } & \mathbf{0}<\mathbf{d}_{1}^{\text {min }} \leq \mathbf{d}_{1} \leq \mathbf{1} \\
& \mathbf{0} \leq \mathbf{d}_{2} \leq \mathbf{1} \\
& V_{e} \sum_{n=1}^{N} d_{1_{n}}-\Theta_{1} \leq 0 \\
& \sum_{n=1}^{N} d_{1_{n}} d_{2_{n}}-\Theta_{2} \sum_{n=1}^{N} d_{1_{n}} \leq 0 \\
& {\left[\begin{array}{ll}
\mathbf{K}_{\mathbf{u u}} & \mathbf{K}_{\mathbf{u} \phi} \\
\mathbf{K}_{\phi \mathbf{u}} & -\mathbf{K}_{\phi \phi}
\end{array}\right]\left\{\begin{array}{l}
\mathbf{U}_{1} \\
\boldsymbol{\Phi}_{1}
\end{array}\right\}=\left\{\begin{array}{l}
\mathbf{F}_{1} \\
\mathbf{Q}_{1}
\end{array}\right\} }
\end{aligned}
$$

onde, $\mathbf{d}_{1}^{\text {min }}$ denota os valores mínimos da classe de variáveis $\mathbf{d}_{1}$, para evitar a singularidade da matriz de rigidez do MEF, e $V_{e}$ é o volume do elemento finito.

\subsubsection{Análise de Sensibilidades}

Como $\mathbf{Z}_{\Gamma_{t}}$ independe das pseudodensidades, derivando a função-objetivo da Equação (3.3) em relação a $\rho_{i}$, tem-se que:

$$
\frac{\partial L_{1}}{\partial \rho_{i}}=\mathbf{Z}_{\Gamma_{t}}^{t} \frac{\partial \mathbf{U}_{1}}{\partial \rho_{i}}
$$

Baseando-se na Teoria da Elasticidade, $\frac{\partial \mathbf{U}_{1}}{\partial \rho_{i}}$ pode ser obtido implicitamente, sabendo-se que:

$$
\begin{gathered}
{\left[\begin{array}{cc}
\mathbf{K}_{\mathbf{u u}} & \mathbf{K}_{\mathbf{u} \phi} \\
\mathbf{K}_{\phi \mathbf{u}} & -\mathbf{K}_{\phi \phi}
\end{array}\right]\left\{\begin{array}{l}
\mathbf{U} \\
\mathbf{\Phi}
\end{array}\right\}=\left\{\begin{array}{l}
\mathbf{F} \\
\mathbf{Q}
\end{array}\right\}} \\
{\left[\begin{array}{cc}
\partial \mathbf{K}_{\mathbf{u u}} & \partial \mathbf{K}_{\mathbf{u} \phi} \\
\partial \mathbf{K}_{\phi \mathbf{u}} & -\partial \mathbf{K}_{\phi \phi}
\end{array}\right]\left\{\begin{array}{c}
\mathbf{U} \\
\mathbf{\Phi}
\end{array}\right\}+\left[\begin{array}{cc}
\mathbf{K}_{\mathbf{u u}} & \mathbf{K}_{\mathbf{u} \phi} \\
\mathbf{K}_{\phi \mathbf{u}} & -\mathbf{K}_{\phi \phi}
\end{array}\right]\left\{\begin{array}{l}
\partial \mathbf{U} \\
\partial \boldsymbol{\Phi}
\end{array}\right\}=\left\{\begin{array}{c}
\partial \mathbf{F} \\
\partial \mathbf{Q}
\end{array}\right\}}
\end{gathered}
$$

onde, por facilidade de notação, $\partial \bullet=\frac{\partial \bullet}{\partial \rho_{i}}$.

Quando os carregamentos independem das variáveis de projeto, tem-se que:

$$
\left\{\begin{array}{c}
\partial \mathbf{U} \\
\partial \boldsymbol{\Phi}
\end{array}\right\}=-\left[\begin{array}{cc}
\mathbf{K}_{\mathbf{u u}} & \mathbf{K}_{\mathbf{u} \phi} \\
\mathbf{K}_{\phi \mathbf{u}} & -\mathbf{K}_{\phi \phi}
\end{array}\right]^{-1}\left[\begin{array}{cc}
\partial \mathbf{K}_{\mathbf{u u}} & \partial \mathbf{K}_{\mathbf{u} \phi} \\
\partial \mathbf{K}_{\phi \mathbf{u}} & -\partial \mathbf{K}_{\phi \phi}
\end{array}\right]\left\{\begin{array}{c}
\mathbf{U} \\
\boldsymbol{\Phi}
\end{array}\right\}
$$

Porém, quando prescrevem-se potenciais elétricos, é preciso considerar o sistema da Equação (B.39), cuja derivada é calculada da seguinte forma: 


$$
\begin{gathered}
{\left[\begin{array}{ccc}
\partial \mathbf{K}_{\mathbf{u u}} & \partial \mathbf{K}_{\mathbf{u} \phi_{l}} & \mathbf{0} \\
\partial \mathbf{K}_{\phi_{l} \mathbf{u}} & -\partial \mathbf{K}_{\phi_{l} \phi_{l}} & \mathbf{0} \\
\mathbf{0} & \mathbf{0} & \mathbf{0}
\end{array}\right]\left\{\begin{array}{c}
\mathbf{U} \\
\boldsymbol{\Phi}_{l} \\
\boldsymbol{\Phi}_{p}
\end{array}\right\}+\left[\begin{array}{ccc}
\mathbf{K}_{\mathbf{u u}} & \mathbf{K}_{\mathbf{u} \phi_{l}} & \mathbf{0} \\
\mathbf{K}_{\phi_{l} \mathbf{u}} & -\mathbf{K}_{\phi_{l} \phi_{l}} & \mathbf{0} \\
\mathbf{0} & \mathbf{0} & \mathbf{I}
\end{array}\right]\left\{\begin{array}{c}
\partial \mathbf{U} \\
\partial \boldsymbol{\Phi}_{l} \\
\mathbf{0}
\end{array}\right\}=\left\{\begin{array}{c}
-\partial \mathbf{K}_{\mathbf{u} \phi_{p}} \boldsymbol{\Phi}_{p} \\
\partial \mathbf{K}_{\phi_{l} \phi_{p}} \boldsymbol{\Phi}_{p} \\
\mathbf{0}
\end{array}\right\}} \\
{\left[\begin{array}{ccc}
\mathbf{K}_{\mathbf{u u}} & \mathbf{K}_{\mathbf{u} \phi_{l}} & \mathbf{0} \\
\mathbf{K}_{\phi_{l} \mathbf{u}} & -\mathbf{K}_{\phi_{l} \phi_{l}} & \mathbf{0} \\
\mathbf{0} & \mathbf{0} & \mathbf{I}
\end{array}\right]\left\{\begin{array}{c}
\partial \mathbf{U} \\
\partial \boldsymbol{\Phi}_{l} \\
\mathbf{0}
\end{array}\right\}=-\left[\begin{array}{ccc}
\partial \mathbf{K}_{\mathbf{u u}} & \partial \mathbf{K}_{\mathbf{u} \phi_{l}} & \partial \mathbf{K}_{\mathbf{u} \phi_{p}} \\
\partial \mathbf{K}_{\phi_{l} \mathbf{u}} & -\partial \mathbf{K}_{\phi_{l} \phi_{l}} & -\partial \mathbf{K}_{\phi_{l} \phi_{p}} \\
\mathbf{0} & \mathbf{0} & \mathbf{0}
\end{array}\right]\left\{\begin{array}{c}
\mathbf{U} \\
\boldsymbol{\Phi}_{l} \\
\boldsymbol{\Phi}_{p}
\end{array}\right\}}
\end{gathered}
$$

Assim, as derivadas dos deslocamentos livres são dadas por:

$$
\left\{\begin{array}{c}
\partial \mathbf{U} \\
\partial \boldsymbol{\Phi}_{l}
\end{array}\right\}=-\left[\begin{array}{cc}
\mathbf{K}_{\mathbf{u u}} & \mathbf{K}_{\mathbf{u} \phi_{l}} \\
\mathbf{K}_{\phi_{l} \mathbf{u}} & -\mathbf{K}_{\phi_{l} \phi_{l}}
\end{array}\right]^{-1}\left[\begin{array}{ccc}
\partial \mathbf{K}_{\mathbf{u u}} & \partial \mathbf{K}_{\mathbf{u} \phi_{l}} & \partial \mathbf{K}_{\mathbf{u} \phi_{p}} \\
\partial \mathbf{K}_{\phi_{l} \mathbf{u}} & -\partial \mathbf{K}_{\phi_{l} \phi_{l}} & -\partial \mathbf{K}_{\phi_{l} \phi_{p}}
\end{array}\right]\left\{\begin{array}{c}
\mathbf{U} \\
\boldsymbol{\Phi}_{l} \\
\boldsymbol{\Phi}_{p}
\end{array}\right\}
$$

Como o domínio piezelétrico é fixo, a Equação (3.9) torna-se:

$$
\left\{\begin{array}{c}
\partial \mathbf{U}_{1} \\
\partial \boldsymbol{\Phi}_{1_{l}}
\end{array}\right\}=-\left[\begin{array}{cc}
\mathbf{K}_{\mathbf{u u}} & \mathbf{K}_{\mathbf{u} \phi_{l}} \\
\mathbf{K}_{\phi_{l} \mathbf{u}} & -\mathbf{K}_{\phi_{l} \phi_{l}}
\end{array}\right]^{-1}\left[\begin{array}{ccc}
\partial \mathbf{K}_{\mathbf{u u}} & \mathbf{0} & \mathbf{0} \\
\mathbf{0} & \mathbf{0} & \mathbf{0}
\end{array}\right]\left\{\begin{array}{c}
\mathbf{U}_{1} \\
\boldsymbol{\Phi}_{1_{l}} \\
\boldsymbol{\Phi}_{1_{p}}
\end{array}\right\}
$$

Devido à inversão da matriz, os graus de liberdade de interesse não podem ser destacados dos demais e, assim, é interessante reescrever a sensibilidade de $L_{1}$ para evitar o cálculo de derivadas não utilizadas:

$$
\frac{\partial L_{1}}{\partial \rho_{i}}=\left\{\begin{array}{ll}
\mathbf{Z}_{\Gamma_{t}}^{t} & \mathbf{0}
\end{array}\right\}\left\{\begin{array}{c}
\partial \mathbf{U}_{1} \\
\partial \boldsymbol{\Phi}_{1}
\end{array}\right\}=-\boldsymbol{\Psi}_{1}^{t}\left[\begin{array}{cc}
\partial \mathbf{K}_{\mathbf{u u}} & \mathbf{0} \\
\mathbf{0} & \mathbf{0}
\end{array}\right]\left\{\begin{array}{c}
\mathbf{U}_{1} \\
\boldsymbol{\Phi}_{1}
\end{array}\right\}
$$

onde, $\Psi_{1}$ é o vetor adjunto obtido pela solução do seguinte sistema:

$$
\left[\begin{array}{cc}
\mathbf{K}_{\mathbf{u u}} & \mathbf{K}_{\mathbf{u} \phi} \\
\mathbf{K}_{\phi \mathbf{u}} & -\mathbf{K}_{\phi \phi}
\end{array}\right] \boldsymbol{\Psi}_{1}=\left\{\begin{array}{c}
\mathbf{Z}_{\Gamma_{t}} \\
\mathbf{0}
\end{array}\right\}
$$

Com isso, as únicas sensibilidades a serem explicitamente calculadas são as dos termos da matriz de rigidez mecânica, sendo que, da Equação (B.34), sabe-se que:

$$
\left[\mathbf{K}_{\mathbf{u u}}\right]_{e}=\int_{V_{e}}\left[\mathbf{B}_{\mathbf{u}}\right]^{t}\left[\mathbf{c}^{E}\right]\left[\mathbf{B}_{\mathbf{u}}\right] d V_{e}
$$


Nesta equação, a matriz $\mathbf{c}^{E}$ do material resultante é ditada pelo MISP, como definido na Equação (B.34), e é dada por:

$$
\mathbf{c}=\left(1-\rho_{1_{e}}^{p}\right) \mathbf{c}_{v a z i o}+\rho_{1_{e}}^{p}\left(\left(1-\rho_{2_{e}}^{q}\right) \mathbf{c}_{\text {mat } 1}+\rho_{2_{e}}^{q} \mathbf{c}_{\text {mat } 2}\right)
$$

onde $\rho_{k_{e}}$ denota a pseudodensidade do elemento, de acordo com a Equação (3.1).

Assim, as sensibilidades em relação às duas classes de variáveis de projeto valem:

$$
\begin{gathered}
\frac{\partial \mathbf{c}}{\partial \rho_{1_{e}}}=-p \rho_{1_{e}}^{p-1} \mathbf{c}_{\text {vazio }}+p \rho_{1_{e}}^{p-1}\left(\left(1-\rho_{2_{e}}^{q}\right) \mathbf{c}_{m a t 1}+\rho_{2_{e}}^{q} \mathbf{c}_{m a t 2}\right) \\
\frac{\partial \mathbf{c}}{\partial \rho_{2_{e}}}=\rho_{1_{e}}^{p}\left(-q \rho_{2_{e}}^{q-1} \mathbf{c}_{\text {mat } 1}+q \rho_{2_{e}}^{q-1} \mathbf{c}_{\text {mat } 2}\right)
\end{gathered}
$$

Finalmente, de posse das derivadas em relação às pseudodensidades, é possível obter as sensibilidades em relação às variáveis de projeto, visto que:

$$
\frac{\partial \mathbf{c}}{\partial d_{k_{i}}}=\frac{\partial \mathbf{c}}{\partial \rho_{k_{e}}} \frac{\partial \rho_{k_{e}}}{\partial d_{k_{i}}}
$$

onde $\frac{\partial \rho_{k_{e}}}{\partial d_{k_{i}}}$ é obtida derivando a Equação (2.6):

$$
\frac{\partial \rho_{k_{e}}}{\partial d_{k_{i}}}=\frac{w_{i e}}{\sum_{j \in S_{i}^{r}} w_{j e}}
$$

\subsection{Otimização de Sensores Piezelétricos}

Para a implementação, via MEF, do problema de otimização formulado na Seção 2.3.2, é necessário reescrevê-lo em termos de graus de liberdade dos elementos finitos do domínio de projeto, como descrito na Seção 3.4.1. Além disso, visto que o PLS se baseia nas séries de Taylor de primeira ordem, também é preciso obter as sensibilidades das funções-objetivo, como é demonstrado na Seção 3.4.2.

\subsubsection{Formulação Discreta}

A função-objetivo para otimização de atuadores piezelétricos, dada pela Equação (2.11), pode ser reescrita no domínio discreto da seguinte forma:

$$
L_{1}=\mathbf{Z}_{\Gamma_{\phi}}^{t} \Phi_{1}
$$


onde $\boldsymbol{\Phi}_{1}$ é o vetor de potenciais elétricos e $\mathbf{Z}_{\Gamma_{\phi}}$ denota um vetor de zeros que assume valor unitário nos graus de liberdade referentes ao potencial elétrico em $\Gamma_{\phi}$.

Portanto, para a implementação do MEF, o problema de otimização de atuadores piezelétricos se torna:

$$
\begin{aligned}
\text { Maximizar : } & \mathcal{F}_{s}=L_{1}=\mathbf{Z}_{\Gamma_{\phi}}^{t} \mathbf{\Phi}_{1} \\
\mathbf{d}_{1}, \mathbf{d}_{2} & \\
\text { tal que : } & \mathbf{0}<\mathbf{d}_{1}^{m i n} \leq \mathbf{d}_{1} \leq \mathbf{1} \\
& \mathbf{0} \leq \mathbf{d}_{2} \leq \mathbf{1} \\
& V_{e} \sum_{n=1}^{N} d_{1_{n}}-\Theta_{1} \leq 0 \\
& \sum_{n=1}^{N} d_{1_{n}} d_{2_{n}}-\Theta_{2} \sum_{n=1}^{N} d_{1_{n}} \leq 0 \\
& {\left[\begin{array}{ll}
\mathbf{K}_{\mathbf{u u}} & \mathbf{K}_{\mathbf{u} \phi} \\
\mathbf{K}_{\phi \mathbf{u}} & -\mathbf{K}_{\phi \phi}
\end{array}\right]\left\{\begin{array}{l}
\mathbf{U}_{1} \\
\boldsymbol{\Phi}_{1}
\end{array}\right\}=\left\{\begin{array}{l}
\mathbf{F}_{1} \\
\mathbf{Q}_{1}
\end{array}\right\} }
\end{aligned}
$$

\subsubsection{Análise de Sensibilidades}

Analogamente ao caso do projeto de atuadores, $\mathbf{Z}_{\Gamma_{\phi}}$ independe das pseudodensidades $\rho_{i}$, e portanto:

$$
\frac{\partial L_{1}}{\partial \rho_{i}}=\mathbf{Z}_{\Gamma_{\phi}}^{t} \frac{\partial \Phi_{1}}{\partial \rho_{i}}
$$

Como o domínio piezelétrico é fixo, segue-se o desenvolvimento da Seção 3.3.2, concluindo-se que:

$$
\frac{\partial L_{1}}{\partial \rho_{i}}=\left\{\begin{array}{cc}
\mathbf{0} & \mathbf{Z}_{\Gamma_{\phi}}^{t}
\end{array}\right\}\left\{\begin{array}{c}
\partial \mathbf{U}_{1} \\
\partial \boldsymbol{\Phi}_{1}
\end{array}\right\}=-\boldsymbol{\Psi}_{1}^{t}\left[\begin{array}{cc}
\partial \mathbf{K}_{\mathbf{u u}} & \mathbf{0} \\
\mathbf{0} & \mathbf{0}
\end{array}\right]\left\{\begin{array}{c}
\mathbf{U}_{1} \\
\boldsymbol{\Phi}_{1}
\end{array}\right\}
$$

porém, neste caso, $\boldsymbol{\Psi}_{1}$ é o vetor adjunto obtido pela solução deste outro sistema:

$$
\left[\begin{array}{cc}
\mathbf{K}_{\mathbf{u u}} & \mathbf{K}_{\mathbf{u} \phi} \\
\mathbf{K}_{\phi \mathbf{u}} & -\mathbf{K}_{\phi \phi}
\end{array}\right] \boldsymbol{\Psi}_{1}=\left\{\begin{array}{c}
\mathbf{0} \\
\mathbf{Z}_{\Gamma_{\phi}}
\end{array}\right\}
$$

Finalmente, as sensibilidades da matriz de rigidez $\mathbf{K}_{\mathbf{u u}}$ são calculadas conforme as Equações (3.15) e (3.16). E, em seguida, as sensibilidades em relação às variáveis de projeto são dadas pelas Equações (3.17) e (3.18) 


\section{Fabricação, Caracterização e Verificação Experimental}

Este Capítulo se destina à descrição do processo de fabricação de dispositivos com gradação funcional de material e subsequente verificação experimental.

\subsection{Sequência de Atividades para a fabricação de dispositivos piezelétricos gradados}

Como sintetiza a Figura 4.1, a sequência de atividades para a fabricação de dispositivos piezelétricos gradados se inicia com um estudo para definição dos materiais a serem utilizados, observando a compatibilidade de suas características de sinterização. Para verificar essa compatibilidade, são realizados testes com a finalidade de se chegar a um conjunto de parâmetros adequado para a sinterização de todos os materiais selecionados. Assim, amostras homogêneas de cada material são sinterizadas para serem submetidas à caracterização por ultrassom, a fim de obter as suas propriedades elásticas (Seção 4.2). Utilizando essas propriedades, obtidas experimentalmente, segue-se com a implementação do modelo de material do MOT para projetar os dispositivos desejados. De posse dos resultados numéricos otimizados de topologia e gradação, são utilizadas as aproximações da Seção 4.3 para definir a proporção em massa de cada material que corresponde às propriedades elásticas desejadas. Assim, misturas homogêneas dos pós dos materiais selecionados são preparadas de acordo com a rigidez desejada para cada camada do dispositivo.

Conforme detalhado na Seção 4.4, um tarugo primitivo é construído pelo empilhamento dessas misturas homogêneas e submetido a um processo de SJP, realizado com o mesmo conjunto de parâmetros das sinterizações das amostras caracterizadas por ultrassom. Em seguida, processos de microusinagem (descritos na Seção 4.4) são utilizados para a fabricação das topologias complexas obtidas nos resultados numéricos e, finalmente, cerâmicas piezelétricas são coladas a essas estruturas gradadas. Resta, então, realizar a verificação experimental do dispositivo final, que, no caso de atuadores, é feita como descrito na Seção 4.5. Já a verificação experimental de sensores não é abordada neste trabalho. 


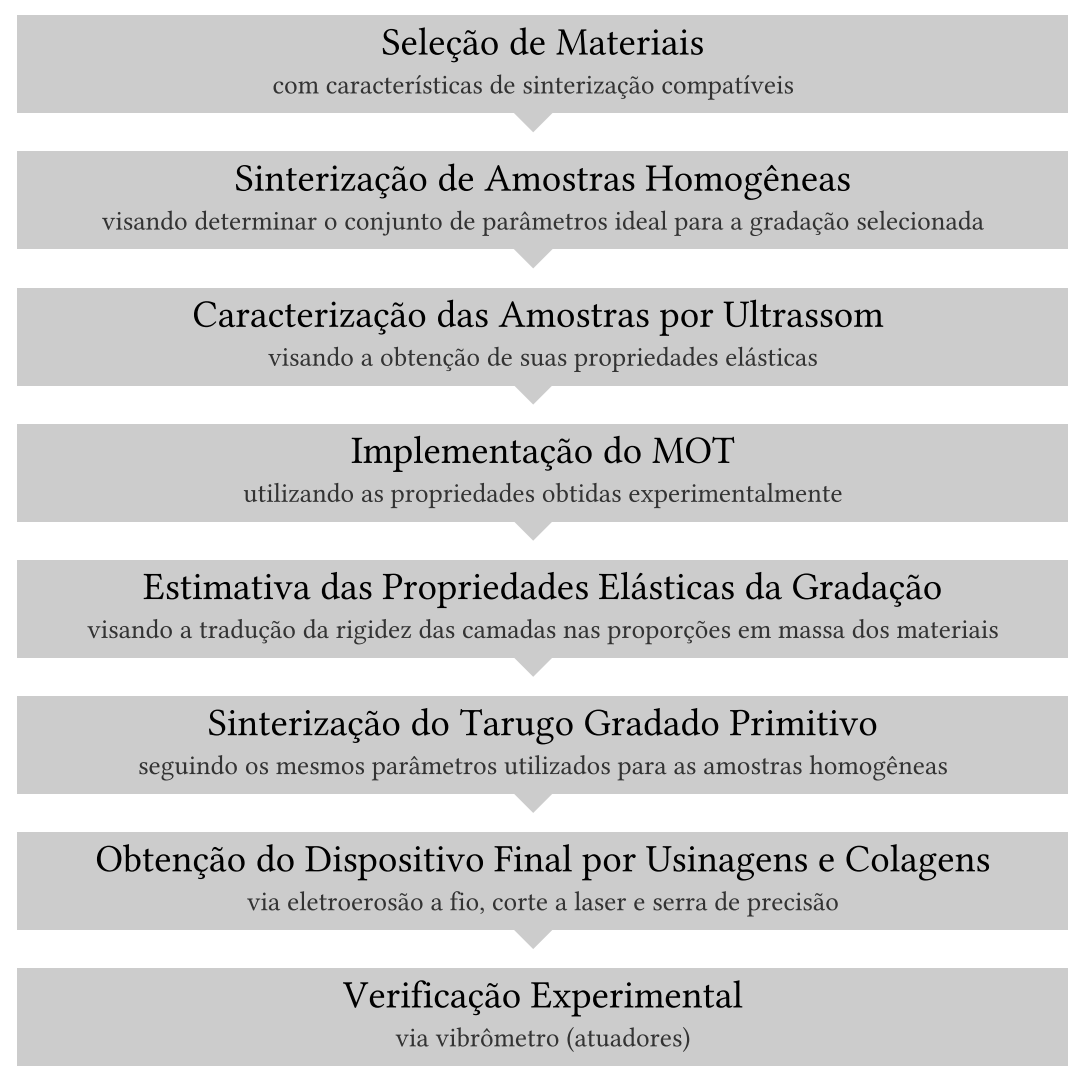

Figura 4.1: Sequência geral de projeto e fabricação de dispositivos piezelétricos gradados.

\subsection{Método Ultrassônico de Caracterização de Materiais}

Para aferir as contantes elásticas de materiais sinterizados, emprega-se um método não-destrutivo baseado na propagação de ondas ultrassônicas (ADAMOWSKI; BUIOCHI; HIGUTI, 2010).

Sabe-se que as propriedades mecânicas elásticas de um material estão relacionadas às velocidades com que as ondas ultrassônicas longitudinais e de cisalhamento se propagam em seu interior. Assim, Wang et al. (2001) estabelece as seguintes relações:

$$
\begin{aligned}
& C_{l}=\sqrt{\frac{M}{\varrho}} \\
& C_{c}=\sqrt{\frac{G}{\varrho}}
\end{aligned}
$$

onde $C_{l}$ e $C_{c}$ são as velocidades de propagação das ondas longitudinais e de cisalhamento no meio, respectivamente. $\varrho$ representa a densidade do material, $M$ denota o módulo de onda de pressão e $G$ denota o módulo de cisalhamento. As demais constantes elásticas podem ser obtidas pelas relações enunciadas no Anexo A. 
Visto que a densidade $\varrho$ é conhecida, resta obter as velocidades de propagação das ondas sonoras no meio. Para isso, uma amostra com faces planas e paralelas é posicionada entre dois transdutores dispostos como nos esquemas da Figura 4.2 e todo o conjunto é imerso em água destilada.

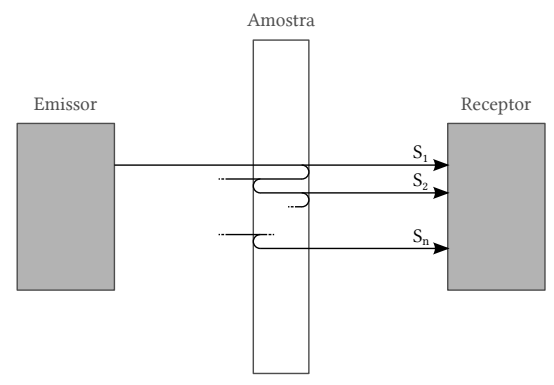

(a)

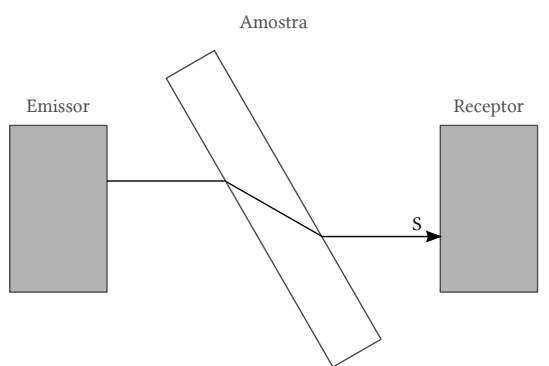

(b)

Figura 4.2: Montagens para medição das velocidades de propagação de ondas (a) longitudinais e (b) de cisalhamento em uma amostra.

Para controlar com precisão o ângulo entre a amostra e os transdutores, é utilizado o goniômetro da Figura 4.3. Neste equipamento, a amostra é fixa a um braço com dois graus de liberdade angulares controlados por motores de passo. Na Figura, o transdutor emissor se encontra à esquerda e o receptor (também conhecido como hidrofone) se encontra à direita, sendo que este último conta com um diâmetro bem maior que o primeiro, devido à sua finalidade de detectar os pulsos emitidos. Para a medição, o transdutor emissor é excitado por um gerador de função e o receptor é monitorado por um osciloscópio.

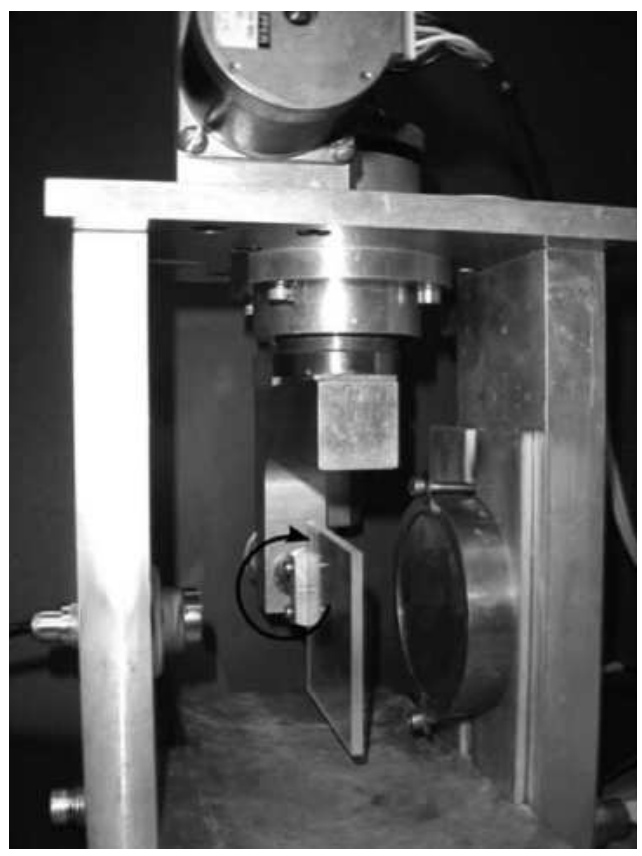

Figura 4.3: Goniômetro para caracterização ultrassônica de amostras de material.

Quando a amostra encontra-se perpendicular às ondas emitidas, como na Figura 4.2(a), 
uma série de picos de intensidade são observados no sinal adquirido. Isso se deve às múltiplas reflexões das ondas nas paredes da amostra, que fazem incidir feixes defasados $\left(S_{1}, S_{2}, S_{3}\right.$ etc.) sobre o receptor. Conhecendo a espessura da amostra e quantificando o tempo de defasagem entre os picos, obtém-se a velocidade de propagação das ondas ultrassônicas longitudinais na amostra $\left(C_{l}\right)$ pela expressão:

$$
C_{l}=(n-1) \frac{2 d}{t_{n}-t_{1}}
$$

onde $d$ denota a espessura da amostra e o tempo médio é calculado pela consideração de $n$ picos de intensidade.

Alternativamente, é possível obter a velocidade $C_{l}$ utilizando a diferença entre o tempo $t_{1}$ da ocorrência do primeiro pico e o tempo $t_{a}$ que a onda toma para percorrer a distância entre os transdutores quando não há nenhum corpo entre eles. Para isso, é preciso conhecer a velocidade $C_{a}$ de propagação das ondas na água destilada - adotada como $1480 \mathrm{~m} / \mathrm{s}$ neste trabalho. Por este método, a velocidade $C_{l}$ é dada por (ANDRADE, 2006):

$$
C_{l}=\frac{C_{a}}{\sqrt{\left(\frac{\left(t_{1}-t_{a}\right) C_{a}}{d}+1\right)^{2}}}
$$

Ao inclinar a amostra além de um ângulo limite, próprio de cada material, os picos de maior intensidade notados pelo receptor são os devidos às ondas cisalhantes - Figura 4.2(b). Neste caso, é necessário realizar duas medições: uma sem nenhum corpo entre os transdutores para obter o tempo $t_{a}$ e outra com a amostra posicionada entre os transdutores e inclinada de um ângulo $\theta$, obtendo o tempo $t_{c}$. Portanto, a velocidade de propagação das ondas ultrassônicas de cisalhamento na amostra $\left(C_{c}\right)$ é dada por (ANDRADE, 2006):

$$
C_{c}=\frac{C_{a}}{\sqrt{\operatorname{sen}^{2} \theta+\left(\frac{\left(t_{c}-t_{a}\right) C_{a}}{d}+\cos \theta\right)^{2}}}
$$

\subsection{Estimativa das Propriedades Elásticas de Gradações Funcionais}

Materiais com gradação funcional apresentam inclusões de um material em uma matriz constituída por outro, ou seja, tratam-se de materiais compósitos. As propriedades efetivas de um material compósito podem ser definidas em termos da energia de deformação que este armazena quando sujeito a uma tensão uniforme. Assim, apoiando-se nos princípios 
variacionais de energia potencial mínima e de energia complementar mínima, Hashin e Shtrikman (1963) estabelecem intervalos de valores para os módulos elásticos desses materiais, considerando que suas fases componentes sejam quasi-isotrópicas.

No caso de um material compósito bifásico, os limites para o valor de seu módulo de compressibilidade $K$ são obtidos considerando os dois casos extremos $K_{\text {inf }}$ e $K_{\text {sup }}$. A curva $K_{\text {inf }}$ é a solução exata do módulo de compressibilidade para um material constituído por uma matriz da fase $A$ com inclusões esféricas da fase $B$ e a curva $K_{\text {sup }}$ é a solução exata para o caso contrário. Suas expressões são dadas, respectivamente, por:

$$
\begin{aligned}
& K_{\text {inf }}\left(v_{B}\right)=K_{A}+\frac{v_{B}}{\frac{1}{K_{B}-K_{A}}+\frac{3\left(1-v_{B}\right)}{3 K_{A}+4 G_{A}}} \\
& K_{\text {sup }}\left(v_{B}\right)=K_{B}+\frac{1-v_{B}}{\frac{1}{K_{A}-K_{B}}+\frac{3 v_{B}}{3 K_{B}+4 G_{B}}}
\end{aligned}
$$

onde $v_{B}$ denota a fração em volume da fase sólida $B$ e $K_{i}$ denota o módulo elástico de compressibilidade e $G_{i}$ denota o módulo elástico de cisalhamento da fase sólida $i$.

Analogamente, o valor efetivo do módulo de cisalhamento $G$, é limitado por $G_{\text {inf }}$ e $G_{\text {sup }}$, cujas expressões são:

$$
\begin{aligned}
G_{\text {inf }}\left(v_{B}\right) & =G_{A}+\frac{v_{B}}{\frac{1}{G_{B}-G_{A}}+\frac{6\left(K_{A}+2 G_{A}\right)\left(1-v_{B}\right)}{5 G_{A}\left(3 K_{A}+4 G_{A}\right)}} \\
G_{\text {sup }}\left(v_{B}\right) & =G_{B}+\frac{1-v_{B}}{\frac{1}{G_{A}-G_{B}}+\frac{6\left(K_{B}+2 G_{B}\right) v_{B}}{5 G_{B}\left(3 K_{B}+4 G_{B}\right)}}
\end{aligned}
$$

O índice das fases que constituem o material compósito são definidas de forma que $K_{A} \leq K_{B}$ e $G_{A} \leq G_{B}$. Dessa forma, $K_{\text {inf }}\left(v_{B}\right) \leq K\left(v_{B}\right) \leq K_{\text {sup }}\left(v_{B}\right)$ e $G_{\text {inf }}\left(v_{B}\right) \leq G\left(v_{B}\right) \leq$ $G_{\text {sup }}\left(v_{B}\right)$, como representado graficamente pela Figura 4.4 .
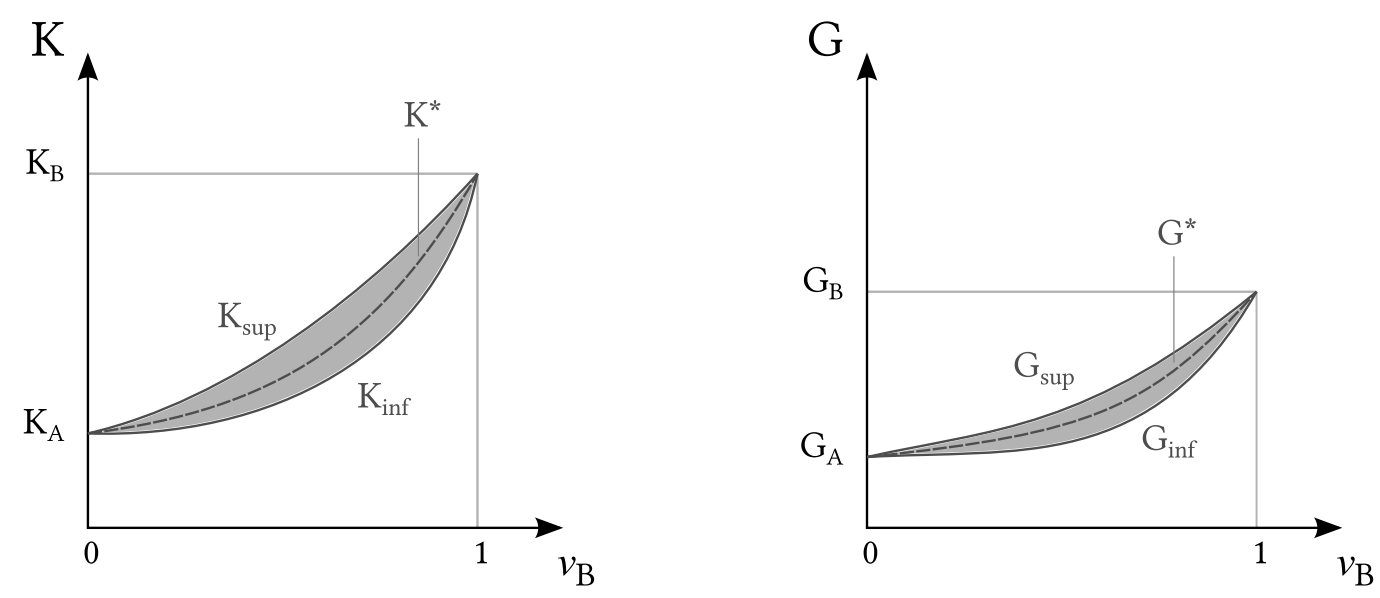

Figura 4.4: Curvas limítrofes do módulo de compressibilidade $K$ e de cisalhamento $G$ em materiais compósitos com fases isotrópicas. 
Como as curvas inferior e superior são geralmente muito próximas (ver Figura 6.10), é possível estimar com boa precisão o valor dos módulos elásticos efetivos do material pela curva média. Ou seja:

$$
\begin{aligned}
K^{*}\left(v_{B}\right) & =\frac{K_{\text {inf }}\left(v_{B}\right)+K_{\text {sup }}\left(v_{B}\right)}{2} \\
G^{*}\left(v_{B}\right) & =\frac{G_{\text {inf }}\left(v_{B}\right)+G_{\text {sup }}\left(v_{B}\right)}{2}
\end{aligned}
$$

Porém, no algoritmo de otimização desenvolvido neste trabalho, a rigidez de uma gradação é variada linearmente com a pseudo-densidade $\rho_{2}$, ou seja:

$$
\begin{aligned}
& K_{\text {alvo }}=K_{A}+\rho_{2}\left(K_{B}-K_{A}\right) \\
& G_{\text {alvo }}=G_{A}+\rho_{2}\left(G_{B}-G_{A}\right)
\end{aligned}
$$

Portanto, para traduzir a rigidez alvo em proporção de materiais, da curva $K^{*}$ se obtém a fração em volume $v_{B}^{K}$ que leva ao módulo de compressibilidade $K_{a l v o}$ e da curva $G^{*}$ se

obtém a fração em volume $v_{B}^{G}$ que leva ao módulo de cisalhamento $G_{a l v o}$. Visto que essas frações em volume são muito próximas (ver Tabela 6.3), é válido aproximar a fração final pela média:

$$
v_{B}^{*}=\frac{v_{B}^{K}+v_{B}^{G}}{2}
$$

\subsection{Processo de Fabricação de Dispositivos com Gradação Funcional de Material}

As topologias ótimas obtidas por este trabalho podem ser manufaturadas a partir de tarugos metálicos gradados sinterizados por SJP, conforme ilustrado na Figura 4.5.

O processo se inicia com a construção do gradiente da estrutura em verde, onde cada camada é composta por uma mistura homogênea de pós. Para isso, o interior do molde deve ser preparado com o revestimento da face do pino inferior (ver Figura 1.4) com uma folha de grafite para evitar a adesão da amostra, o que danificaria ambos. Em seguida, repetem-se os passos de preenchimento do molde com pó e de pré-compactação para cada camada. Por fim, a face do pino superior, assim como a inferior, também deve ser revestida com uma folha de grafite. Essa montagem é, então, submetida à SJP, consolidando a peça através de aquecimento e pressão mecânica. Neste trabalho, o equipamento utilizado para Sinterização por Jato de Plasma (SJP) é o SPS-1050 (SPS SYNTEX INC., 2008), que é mostrado na Figura 

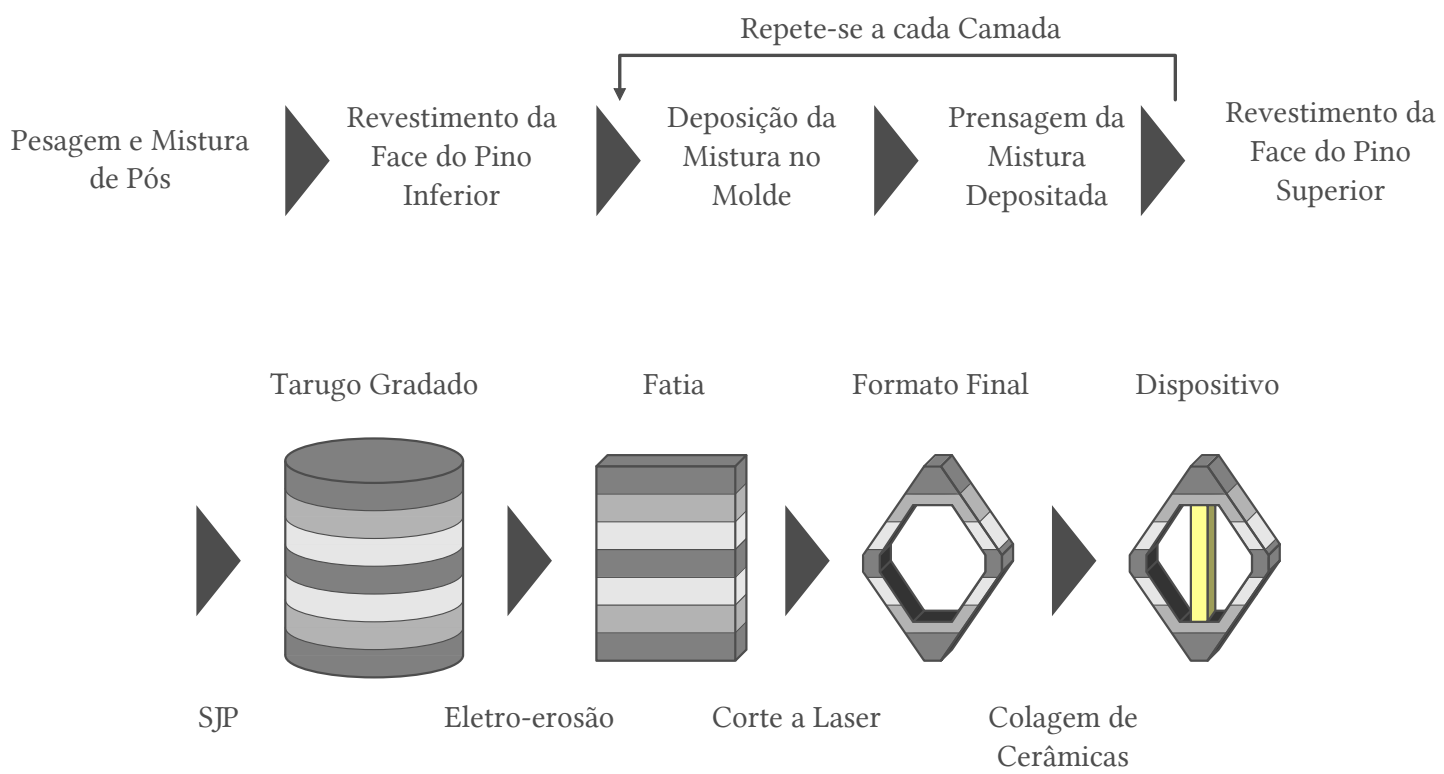

Figura 4.5: Processo de fabricação de dispositivos piezelétricos gradados.

4.6.

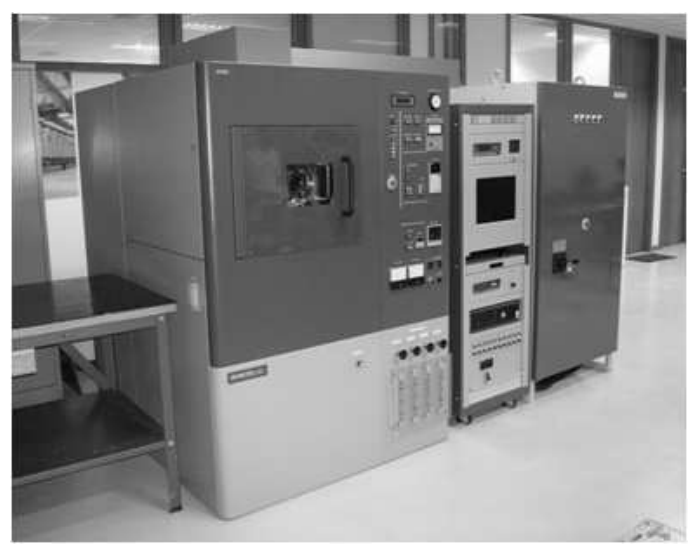

Figura 4.6: SPS-1050 (SPS SYNTEX INC., 2008).

O formato cilíndrico das peças sinterizadas é interessante para a qualidade e homogeneidade das propriedades dos materiais, já que leva a uma distribuição uniforme de temperatura e evita concentrações de tensão. Porém, este formato restringe as aplicações possíveis. Assim, para obter topologias mais complexas é preciso submeter os tarugos sinterizados a processos posteriores de usinagem. Nesta etapa, a Eletro-Erosão a Fio (EEF) e o Corte a Laser (CL) podem ser utilizados individualmente ou em conjunto de acordo com as limitações de cada técnica (MITSUBISHI EDM LASER, 2012). De modo geral, a EEF é mais adequada ao corte de peças mais espessas e o CL a usinagens micrométricas (WIRE CUT COMPANY, 2012; KJ LASER, 2012; WORKSHOP OF PHOTONICS, 2012; INDUSTRIAL LASER SOLUTIONS, 2012).

Por fim, é necessária uma etapa de colagem das cerâmicas às estruturas acopladas usinadas por EEF e CL. Para que as cerâmicas adquiram o formato desejado, elas são 
usinadas previamente por uma serra de precisão ou pelo próprio CL.

\subsection{Método de Verificação Experimental de Atuadores Piezelétricos}

Para determinação do deslocamento gerado na face de atuação dos protótipos fabricados, utiliza-se um vibrômetro OFV-5000 (POLYTEC, 2011). Para isso, os protótipos têm suas cerâmicas excitadas por um gerador de funções. Simplificadamente, o funcionamento do vibrômetro se baseia no princípio de Doppler, medindo a difusão de um laser (classe II He-Ne) incidente em uma superfície, como demonstra o esquema da Figura 4.7. A montagem utilizada nas medições pode ser vista na Figura 4.8, onde o atuador é preso por uma morsa fixada em uma mesa de granito.

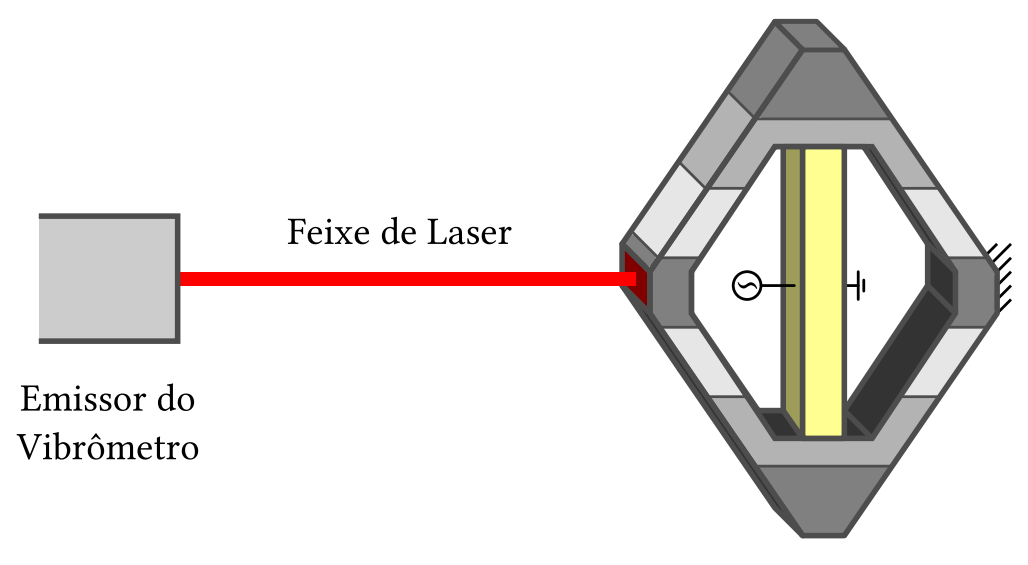

Atuador Piezelétrico

Figura 4.7: Esquema de verificação experimental de protótipo via vibrômetro.

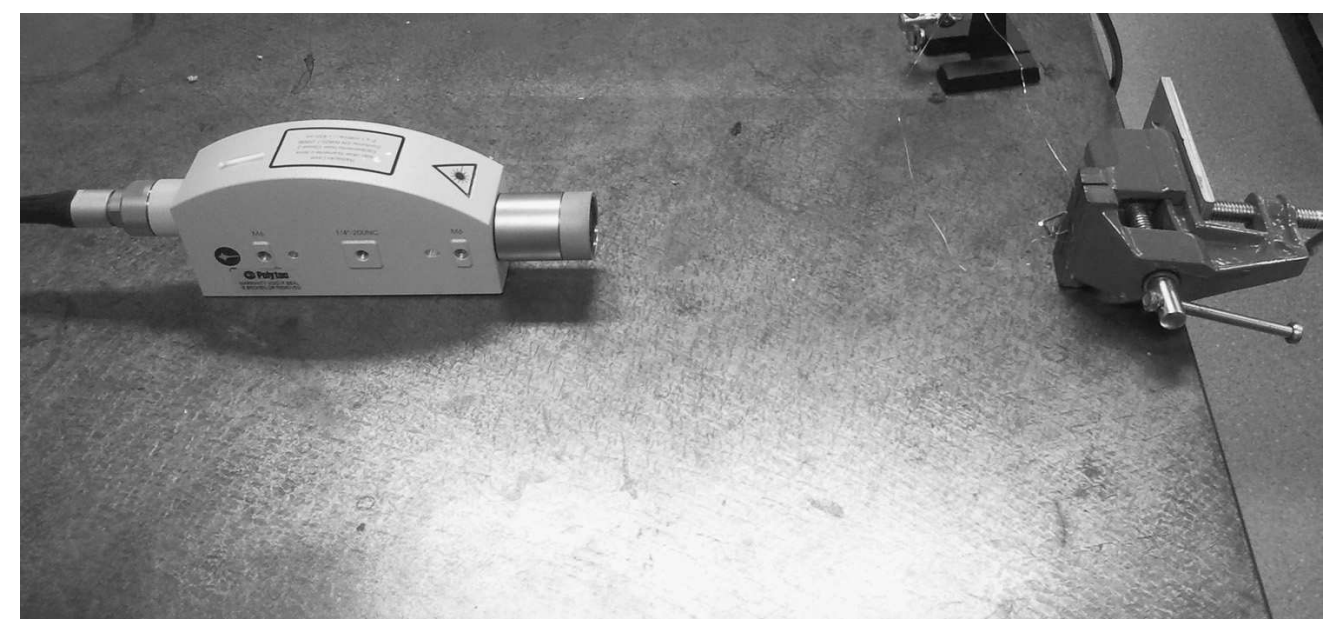

Figura 4.8: Montagem para verificação experimental de protótipo via vibrômetro.

Dada uma excitação senoidal pelo gerador de funções, sabe-se que a resposta de deslocamento tem a seguinte forma: 


$$
s=A_{s} \operatorname{sen}(\omega t+\varphi)
$$

onde, $A_{s}$ denota a amplitude de deslocamento, $\varphi$ a fase angular e $\omega=2 \pi f$ a velocidade angular, sendo $f$ a frequência.

Pela derivada no tempo da resposta de deslocamento, sabe-se que a resposta de velocidade tem a forma:

$$
v=A_{s} \omega \cos (\omega t+\varphi)=A_{v} \cos (\omega t+\varphi)
$$

onde, $A_{v}$ denota a amplitude de velocidade.

Como a resposta do vibrômetro é fornecida em $[\mathrm{V}]$, o sinal de deslocamento em $[\mathrm{mm}]$ e o sinal de velocidade em $[\mathrm{mm} / \mathrm{s}]$ são calculados com base nas sensibilidades $\varsigma_{s}$ e $\varsigma_{v}$ do sensor, respectivamente. Assim, utilizando o decodificador de deslocamentos do equipamento, a amplitude $A_{s}$ em $[\mathrm{mm}]$ é obtida diretamente, pela seguinte expressão:

$$
A_{s}[m m]=A_{s}[V] \cdot \varsigma_{s}[m m / V]
$$

Também é possível obter os deslocamentos indiretamente, derivando o sinal do decodificador de velocidades:

$$
A_{s}[m m]=\frac{A_{v}[V] \cdot \varsigma_{v}[m m / s / V]}{2 \pi \cdot f[H z]}
$$




\section{Resultados Numéricos e Discussões}

Este Capítulo apresenta os resultados numéricos obtidos pelo software de otimização de dispositivos piezelétricos gradados desenvolvido.

\subsection{Moonie Piezelétrico}

Nesta Seção são apresentados os resultados da otimização de um atuador piezelétrico que transforma deslocamentos horizontais de uma cerâmica piezelétrica em deslocamentos verticais, amplificando-os. Neste tipo de dispositivo, conhecido como moonie, o objetivo é maximizar o deslocamento na região $\Gamma_{t}$, baseando-se no domínio de projeto da Figura 5.1 - equivalente a um quadrante do dispositivo completo. Na representação, $\Omega_{p}$ é o domínio cerâmico não otimizável, $\Omega_{s}$ é o domínio metálico otimizável e $\Gamma_{\varphi}$ denota o eletrodo em que se excita a cerâmica por potencial elétrico. O domínio do problema apresentado é discretizado em uma malha de $80 \times 48$ elementos para proceder com a otimização enunciada na Equação (3.4). Considera-se $\Omega_{s}$ constituída por Cobre e Níquel, cujas propriedades elásticas são obtidas experimentalmente e apresentadas na Tabela 6.2. Em $\Omega_{p}$, são consideradas as propriedades do PZT-5A do Anexo B.

O valor de rigidez da mola $k_{\text {mola }}$ é estabelecido em $1 k N / m m$ e se adota $\mathbf{d}_{1}^{\text {min }}=10^{-6}$. Para as variáveis topologia, o coeficiente de penalização é variado de 1 a 3, seguindo o Método da Continuação (MC), e é utilizada a projeção dada pela Equação (2.6) com grau $g=2$ e $R_{\max }$ equivalente ao lado de 4 elementos.

\subsubsection{Otimização com Restrições de Volume e Manufatura}

Nesta Seção, o problema de otimização enunciado pela Equação (3.4) é implementado com restrição de volume $\Theta_{1}=0,2 V_{\Omega_{s}}$ e restrição da gradação em camadas com espessura de $1 \mathrm{~mm}$, visando a manufaturabilidade. Neste problema, as variáveis de topologia $\left(\rho_{1}\right)$ são inicializadas com valor de 0,1 e as de gradação $\left(\rho_{2}\right)$ com valor de 0,5 . 


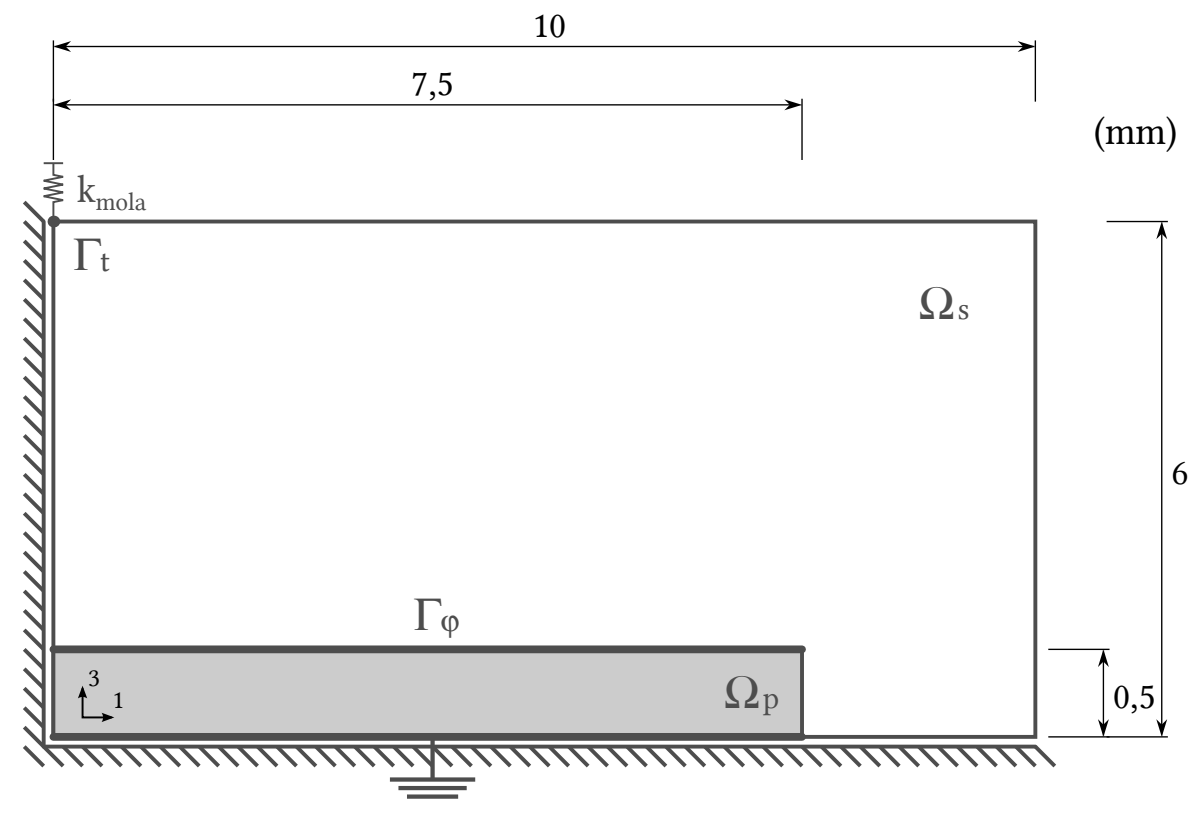

Figura 5.1: Domínio de projeto de moonie piezelétrico (dimensões em $m m$ ).

Porém, com a imposição desta última restrição, surge um problema de condicionamento numérico devido às ordens de grandeza diferentes das variáveis de projeto de topologia e de gradação, já que as pseudodensidades de gradação de todos os nós de uma camada são representadas por uma única variável de projeto $d_{2_{i}}$. Com isso, ocorre uma grande disparidade entre as sensibilidades dessas duas classes de variáveis que dificulta a solução do problema de otimização pelo PLS. Assim, intercalam-se sequências de iterações otimizando apenas a topologia (com gradação constante) com sequências otimizando apenas a gradação (com topologia constante).

O resultado obtido através do MOT possui regiões cinzas, conforme evidenciam as Figuras 5.2(a) e 5.2(c), que apresentam as topologias obtidas para os casos de Cobre e Níquel homogêneos. Essas topologias necessitam de um pós-processamento que estabelece uma densidade de corte para a definição da presença de material - adotada como igual a 0,7 nestes casos. Assim, os formatos finais dos atuadores, considerando a simetria, são apresentados nas Figuras 5.2(b) e 5.2(d).

As Figuras 5.3(a) e 5.3(c) apresentam os resultados para os casos com gradação em camadas perpendiculares e paralelas à cerâmica, cujas pseudodensidades são obtidas pelo MOT. Após o processamento dos resultados (com densidade de corte igual a 0,7), são obtidas as formas finais dos atuadores apresentadas na Figura 5.3, onde as camadas mais claras se aproximam das propriedades elásticas do Cobre e as mais escuras das do Níquel. A região preenchida pelo padrão circular representa a cerâmica PZT-5A. As pseudodensidades de gradação $\rho_{2}$ são dadas na Tabela 5.1 e representadas graficamente na Figura ??.

Os deslocamentos estáticos verticais gerados na face de atuação dos atuadores, devido 


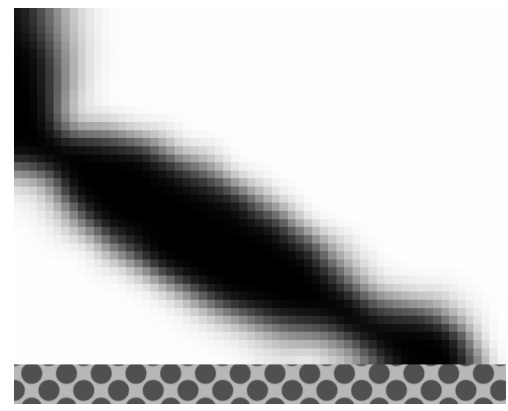

(a) Topologia do caso de Cobre;

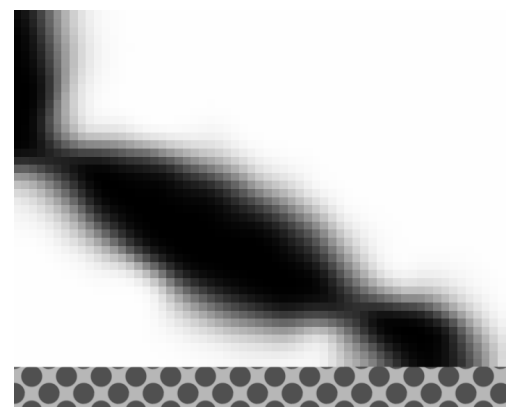

(c) Topologia do caso de Níquel;

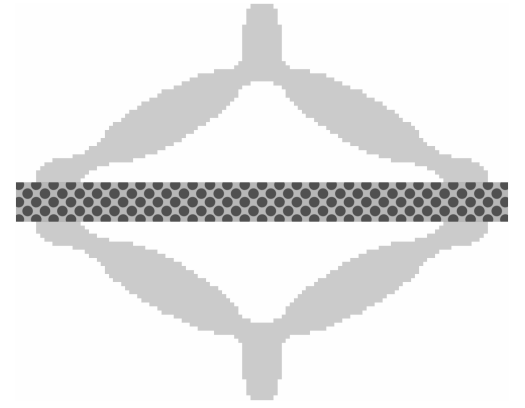

(b) Topologia pós-processada do caso de Cobre;

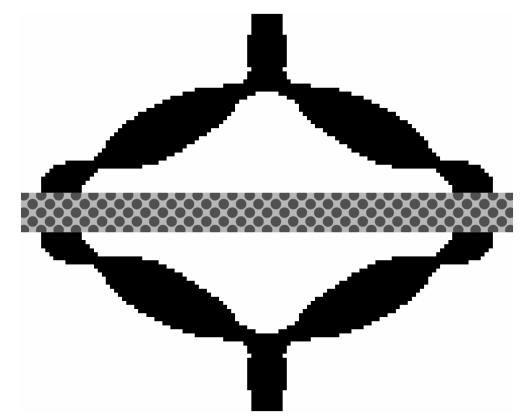

(d) Topologia pós-processada do caso de Níquel.

Figura 5.2: Atuadores, sujeitos a restrições, com estrutura acoplada homogênea.

Tabela 5.1: Pseudodensidades de gradação dos atuadores otimizados.

\begin{tabular}{ccc}
\hline Camada & Figura 5.3(b) & Figura 5.3(d) \\
\hline 1 & 0,0629 & 0,9529 \\
2 & 0,8430 & 0,0705 \\
3 & 0,0152 & 0,2067 \\
4 & 0,9485 & 0,5421 \\
5 & 0,0355 & 0,0043 \\
6 & 0,0020 & 0,0066 \\
7 & 0,4749 & \\
8 & 0,1357 & \\
\hline
\end{tabular}

à aplicação $-100 \mathrm{~V}$ ao eletrodo livre da cerâmica, são apresentados na Tabela 5.2. Esses deslocamentos são exemplificados na Figura 5.5, que apresenta a deformada de um quadrante do atuador Cobre-Níquel com gradação em camadas perpendiculares à cerâmica. Os demais casos possuem deformadas praticamente idênticas a esta, devido à similaridade entre as suas geometrias. 
12233455678
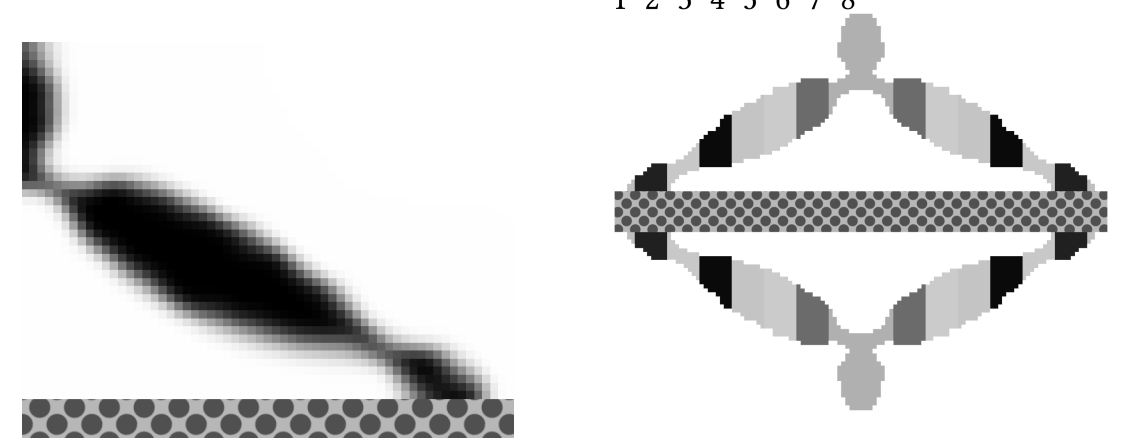

(a) Topologia do caso de camadas (b) Topologia pós-processada e perpendiculares; gradação do caso de camadas perpendiculares;
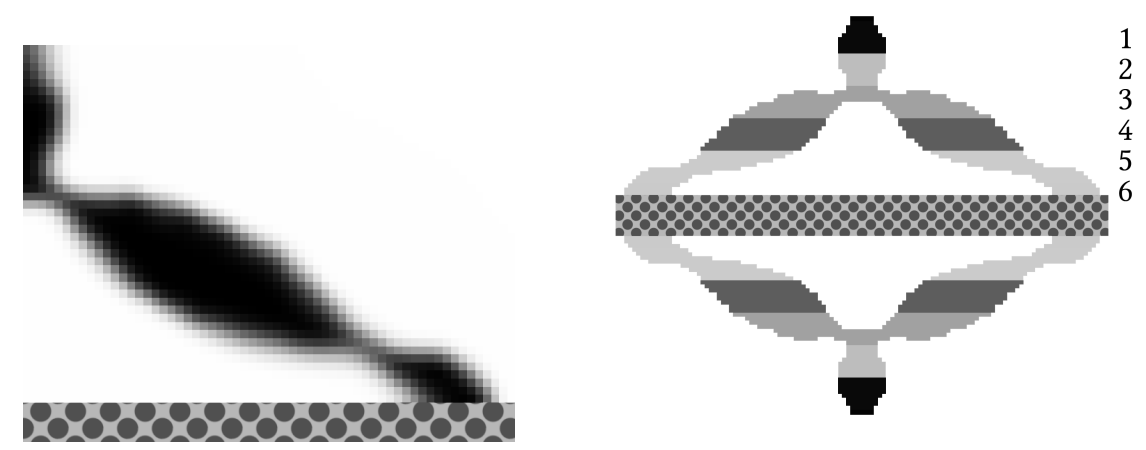

(c) Topologia do caso de camadas (d) Topologia pós-processada e paralelas; gradação do caso de camadas paralelas.

Figura 5.3: Atuadores, sujeitos a restrições, com gradação funcional Cobre-Níquel restrita em camadas.

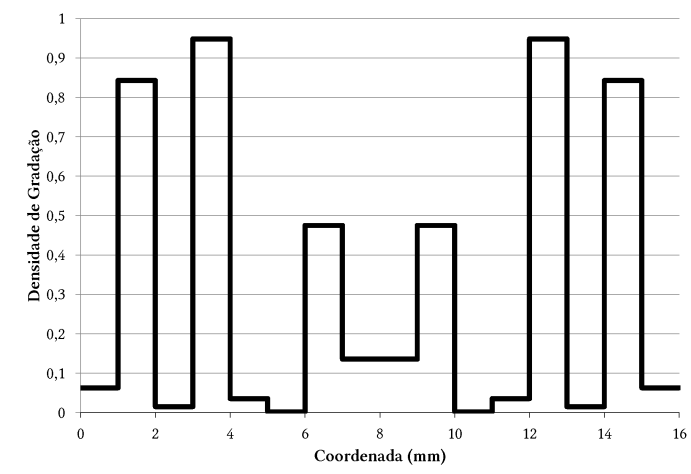

(a) Gradação em camadas perpendiculares à (b) Gradação em camadas paralelas à cerâmica. cerâmica;

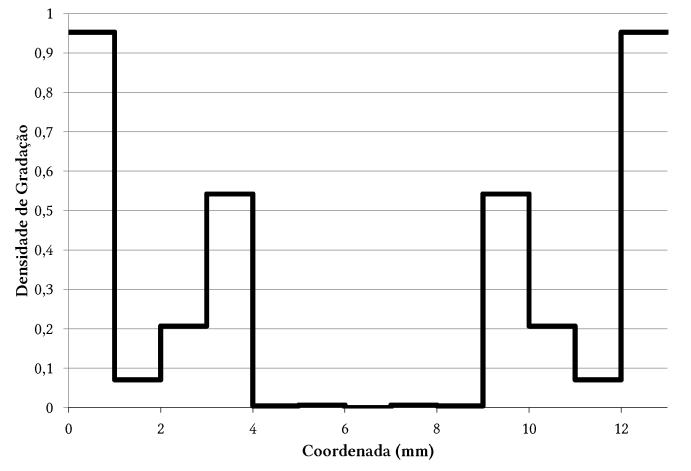

Figura 5.4: Distribuição da gradação nos atuadores otimizados.

\subsubsection{Otimização sem Restrições de Volume e Manufatura}

Nesta Seção, o problema de otimização enunciado pela Equação (3.4) é implementado sem restrição de volume, ou seja, com $\Theta_{1}=V_{\Omega_{s}}$. Além disso, não são impostas restrições 
Tabela 5.2: Deslocamentos estáticos verticais gerados pelos atuadores otimizados sujeitos a restrições.

\begin{tabular}{ccc}
\hline Figura & Resultado do MOT $(\mathrm{nm})$ & Pós-Processado $(\mathrm{nm})$ \\
\hline $5.2(\mathrm{a})$ - Cobre & 101,46 & 109,59 \\
$5.2(\mathrm{c})$ - Níquel & 114,86 & 123,95 \\
$5.3(\mathrm{a})$ - MGF & 120,94 & 126,80 \\
$5.3(\mathrm{c})-\mathrm{MGF}$ & 120,49 & 126,89 \\
\hline
\end{tabular}

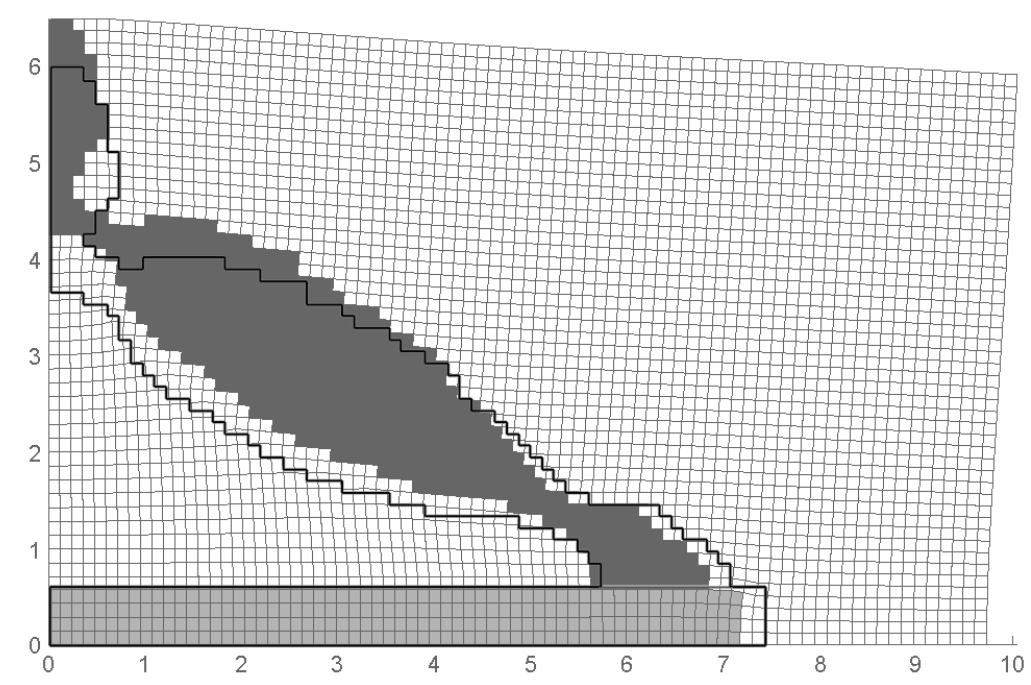

Figura 5.5: Deformada do atuador, sujeito a restrições, com gradação Cobre-Níquel em camadas perpendiculares.

de gradação em camadas, permitindo que o material seja distribuído livremente dentro da estrutura. Isto é feito para avaliar a influência das restrições impostas na Seção anterior no resultado final obtido. Neste problema, as variáveis de topologia $\left(\rho_{1}\right)$ são inicializadas com valor de 0,5 e as de gradação $\left(\rho_{2}\right)$ com valor de 0,5 .

O resultado obtido através do MOT possui regiões cinzas, conforme evidenciam as Figuras 5.6(a) e 5.6(c), que apresentam as topologias obtidas para os casos de Cobre e Níquel homogêneos. Essas topologias necessitam de um pós-processamento que estabelece uma densidade de corte para a definição da presença de material - adotada como igual a 0,7 nestes casos. Assim, os formatos finais dos atuadores, considerando a simetria, são apresentados nas Figuras 5.6(b) e 5.6(d).

Ao incluir a gradação livre de Cobre-Níquel no domínio otimizável, é obtida a topologia da Figura 5.7(a), na qual é utilizada projeção, dada pela Equação (2.6), para as variáveis de gradação $\left(\rho_{2}\right)$ com grau $g=1$ e $R_{\max }$ equivalente ao lado de 12 elementos. Após o processamento deste resultado (com densidade de corte igual a 0,7 ), chega-se à forma final 


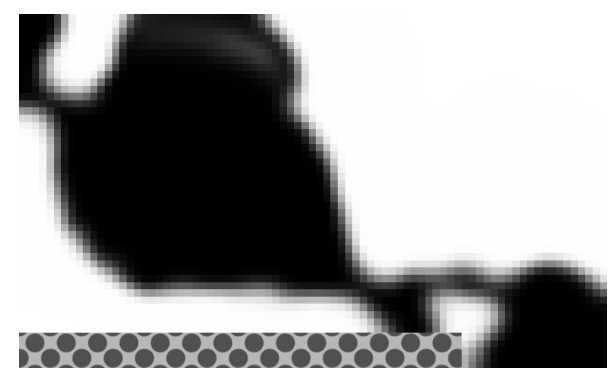

(a) Topologia do caso de Cobre;

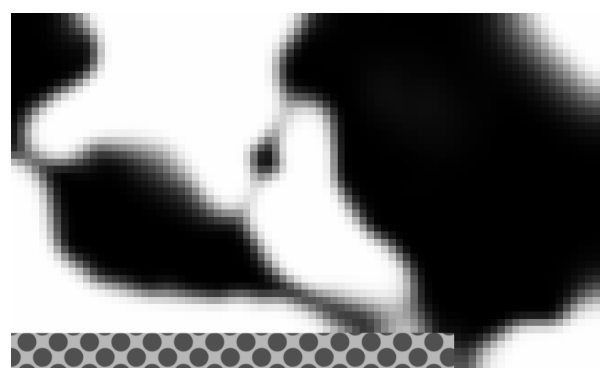

(c) Topologia do caso de Níquel;

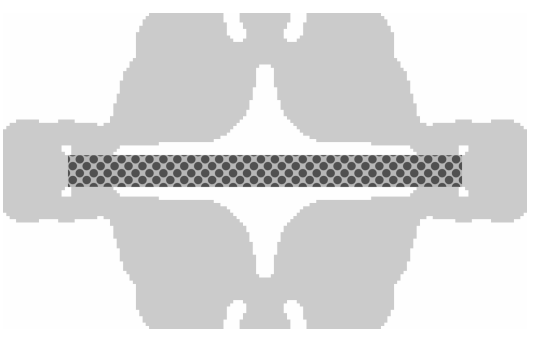

(b) Topologia pós-processada do caso de Cobre;

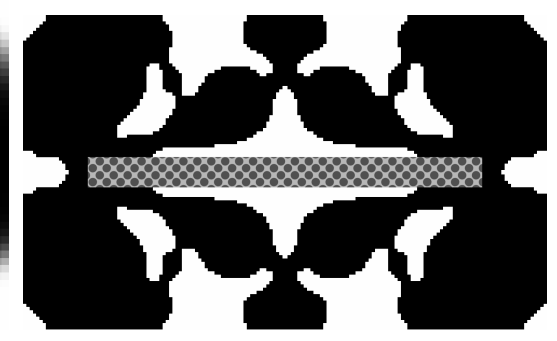

(d) Topologia pós-processada do caso de Níquel.

Figura 5.6: Atuadores com estrutura acoplada homogênea.

do atuador, apresentada na Figura 5.7(b), onde as regiões mais claras se aproximam das propriedades elásticas do Cobre e as mais escuras das do Níquel.

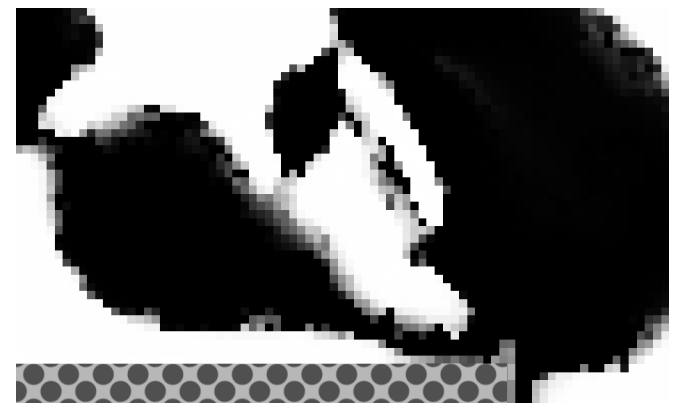

(a) Topologia do caso gradado;

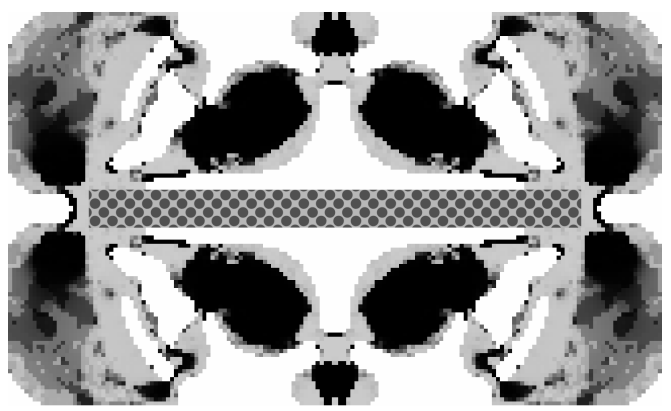

(b) Topologia pós-processada e gradação do caso gradado;

Figura 5.7: Atuador com gradação funcional livre de Cobre-Níquel.

Os deslocamentos estáticos verticais gerados na face de atuação dos atuadores, devido à aplicação $-100 \mathrm{~V}$ ao eletrodo livre da cerâmica, são apresentados na Tabela 5.3. Esses deslocamentos são ilustrados na Figura 5.8, que apresenta a deformada de cada caso dos atuadores otimizados. 
Tabela 5.3: Deslocamentos estáticos verticais gerados pelos atuadores otimizados.

\begin{tabular}{ccc}
\hline Figura & Resultado do MOT $(\mathrm{nm})$ & Pós-Processado $(\mathrm{nm})$ \\
\hline $5.6(\mathrm{a})$ - Cobre & 112,28 & 114,20 \\
$5.6(\mathrm{c})$ - Níquel & 115,06 & 116,88 \\
$5.7(\mathrm{a})$ - MGF & 131,10 & 135,07 \\
\hline
\end{tabular}

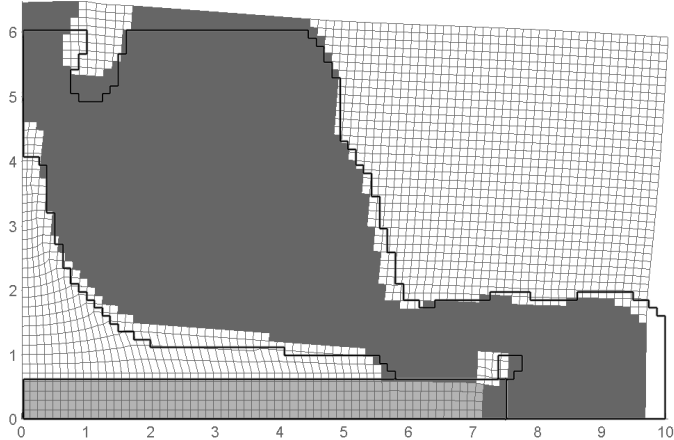

(a) Caso de Cobre homogêneo;

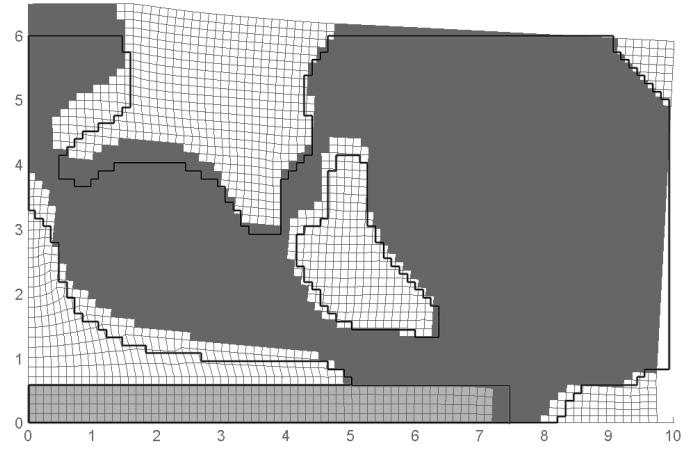

(b) Caso de Níquel homogêneo;

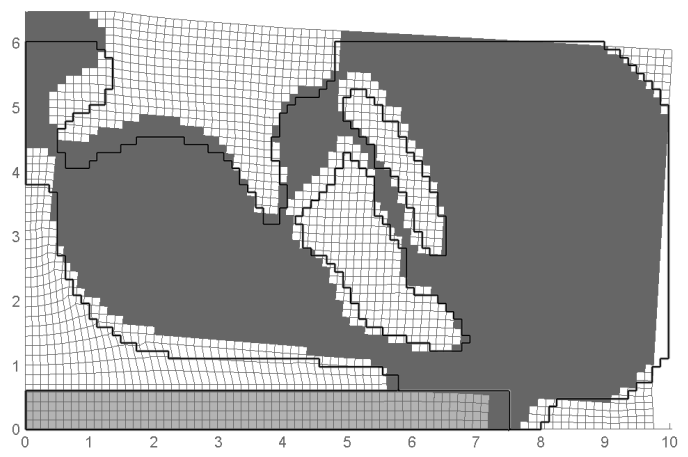

(c) Caso de gradação Cobre-Níquel;

Figura 5.8: Deformadas dos atuadores otimizados.

\subsubsection{Discussão}

Ao incluir a otimização da gradação de material, fica evidente a concentração de material mais flexível nas regiões em que as maiores deformações são esperadas, as chamadas hinges.

Em relação ao objetivo do projeto, os resultados sem restrições de volume e manufatura (Seção 5.1.2) mostram que ao utilizar a gradação funcional Cobre-Níquel o deslocamento de saída é 15,6\% superior ao melhor caso homogêneo (Níquel), como evidenciado na Tabela 5.3 .

O fato do caso homogêneo de Níquel possuir deslocamento maior do que o caso 
homogêneo de Cobre condiz com o esperado, já que um material mais rígido tem condições de causar maiores deformações na mola, que foi imposta como condição de contorno para garantia da rigidez da estrutura otimizada. Tendo em vista a aplicação deste tipo de dispositivo, a inclusão da mola reproduz a condição de contorno real, já que um atuador deve ser capaz de deslocar elementos do mecanismo que atua, vencendo forças contrárias.

Quando se impõem restrições (Seção 5.1.1), as estruturas gradadas apresentam resultados levemente superiores aos dos casos homogêneos. Entre os homogêneos, o maior deslocamento é observado na estrutura em Níquel: 123,95nm. No comparativo, as estruturas gradadas apresentam deslocamentos cerca de 2,4\% superiores ao melhor caso homogêneo, tanto para gradação paralela quanto perpendicular à cerâmica.

A utilização de camadas relativamente espessas impõe uma forte restrição ao problema, contudo, ao restringir o problema em camadas com a espessura de apenas um elemento, pouca melhora em relação ao valor da função objetivo foi alcançada.

\subsection{Garra Piezelétrica}

Nesta Seção são apresentados os resultados da otimização de uma garra piezelétrica, onde o objetivo é maximizar o deslocamento de abertura da garra (deslocamento horizontal médio dos nós pertencentes à região $\Gamma_{t}$ ), baseando-se no domínio de projeto da Figura 5.9, que considera a simetria horizontal do dispositivo. Na representação, $\Omega_{p}$ é o domínio cerâmico não otimizável, $\Omega_{s}$ é o domínio metálico otimizável e $\Gamma_{\varphi}$ denota o eletrodo em que se excita a cerâmica por potencial elétrico. O domínio do problema apresentado é discretizado em uma malha de $80 \times 80$ elementos para proceder com a otimização enunciada na Equação (3.4). Considera-se $\Omega_{s}$ constituída por Cobre e Níquel, cujas propriedades elásticas são obtidas experimentalmente e apresentadas na Tabela 6.2. Em $\Omega_{p}$, são consideradas as propriedades do PZT-5A do Anexo B.

O valor de rigidez da mola $k_{\text {mola }}$ é estabelecido em $1 k N / m m$ e se adota $\mathbf{d}_{1}^{\text {min }}=10^{-6}$. Para as variáveis topologia, o coeficiente de penalização é variado de 1 a 7 , seguindo o MC, e é utilizada a projeção dada pela Equação (2.6) com grau $g=2$ e $R_{\max }$ equivalente ao lado de 4 elementos.

\subsubsection{Otimização com Restrições de Volume e Manufatura}

Nesta Seção, o problema de otimização enunciado pela Equação (3.4) é implementado com restrição de volume $\Theta_{1}=0,3 V_{\Omega_{s}}$ e restrição da gradação em camadas com espessura de $1 \mathrm{~mm}$, visando a manufaturabilidade. Porém, com a imposição desta restrição de manufatura, 


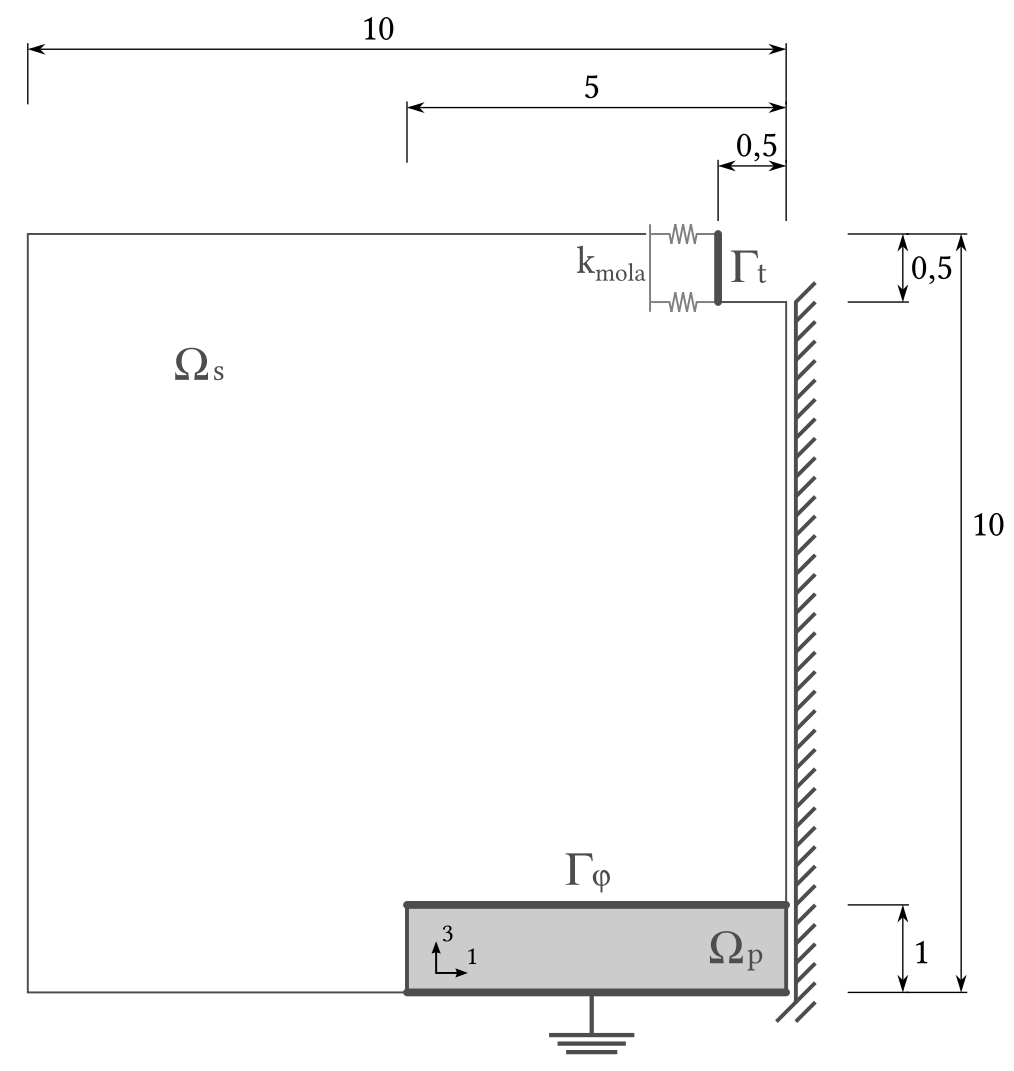

Figura 5.9: Domínio de projeto de garra piezelétrica (dimensões em $\mathrm{mm}$ ).

surge um problema de condicionamento numérico que é contornado da forma detalhada na Seção 5.1.1. Nos resultados apresentados a seguir, as variáveis de topologia $\left(\rho_{1}\right)$ são inicializadas com valor de 0,15 e as de gradação $\left(\rho_{2}\right)$ com valor de 0,5 .

O resultado obtido através do MOT possui regiões cinzas, conforme evidenciam as Figuras 5.10(a) e 5.10(c), que apresentam as topologias obtidas para os casos de Cobre e Níquel homogêneos. Essas topologias necessitam de um pós-processamento que estabelece uma densidade de corte para a definição da presença de material - adotada como igual a 0,6 nestes casos. Assim, os formatos finais das garras, considerando a simetria, são apresentados nas Figuras 5.10(b) e 5.10(d).

As Figuras 5.11(a) e 5.11(c) apresentam os resultados para os casos com gradação em camadas perpendiculares e paralelas à cerâmica, cujas pseudodensidades são obtidas pelo MOT. Após o processamento dos resultados (com densidade de corte igual a 0,6), são obtidas as formas finais das garras apresentadas nas Figuras 5.11(b) e 5.11(d), onde as camadas mais claras se aproximam das propriedades elásticas do Cobre e as mais escuras das do Níquel. As pseudodensidades de gradação $\rho_{2}$ são dadas na Tabela 5.4 e representadas graficamente na Figura ??.

Os deslocamentos estáticos horizontais médios dos nós pertencentes à região $\Gamma_{t}$ das garras, devido à aplicação $-100 \mathrm{~V}$ ao eletrodo livre da cerâmica, são apresentados na Tabela 


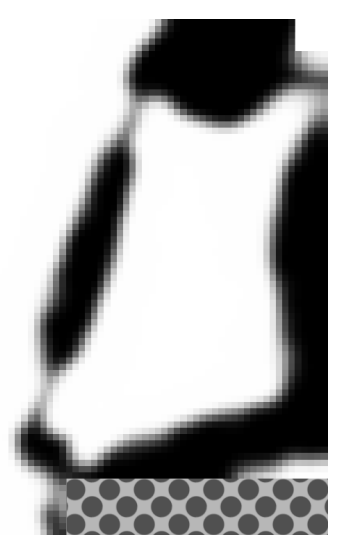

(a) Topologia do caso de Cobre;

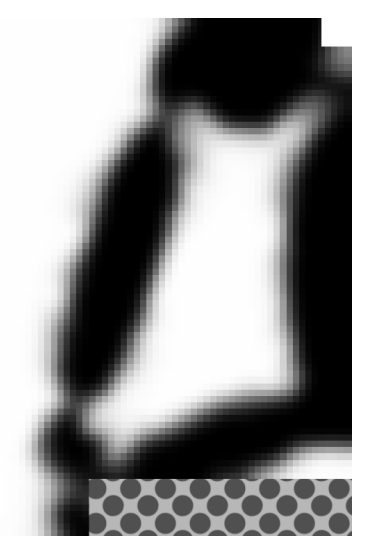

(c) Topologia do caso de Níquel;

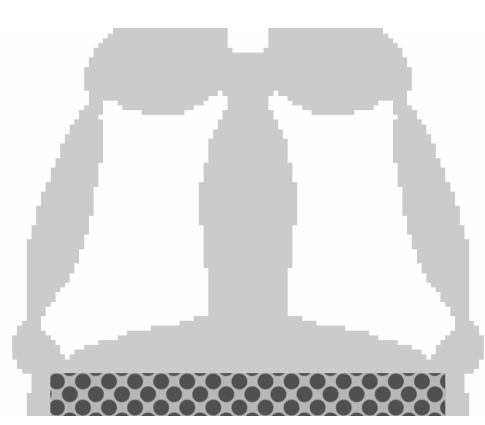

(b) Topologia pós-processada do caso de Cobre;

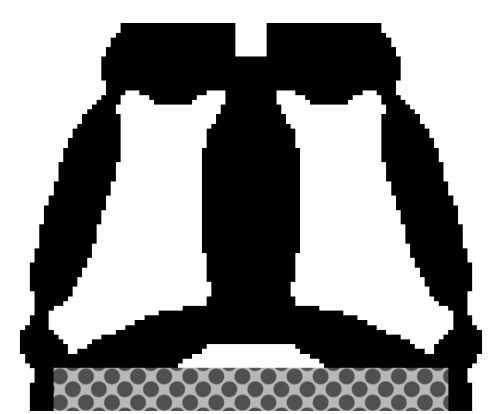

(d) Topologia pós-processada do caso de Níquel.

Figura 5.10: Garras, sujeitas a restrições, com estrutura acoplada homogênea.

Tabela 5.4: Pseudodensidades de gradação das garras otimizadas sujeitas a restrições.

\begin{tabular}{ccc}
\hline Camada & Figura 5.11(b) & (b) Figura 5.11(d) \\
\hline 1 & 0,0000 & 0,9955 \\
2 & 0,0121 & 0,0121 \\
3 & 0,0828 & 0,7516 \\
4 & 0,8684 & 0,3715 \\
5 & 0,9626 & 0,0746 \\
6 & 0,0004 & 0,0264 \\
7 & & 0,0591 \\
8 & & 0,1166 \\
9 & & 0,0000 \\
10 & & 0,0006 \\
\hline
\end{tabular}

5.5. Esses deslocamentos são ilustrados na Figura 5.13, que apresenta a deformada de cada caso das garras otimizadas. 


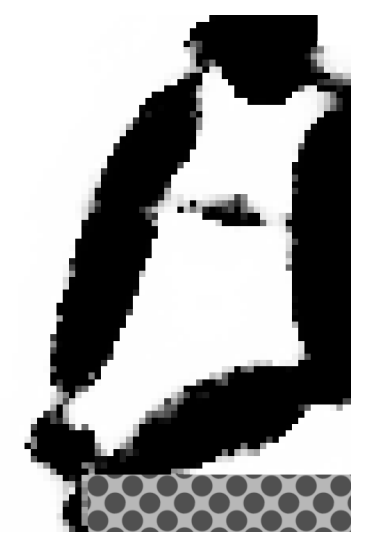

(a) Topologia do caso de camadas perpendiculares;

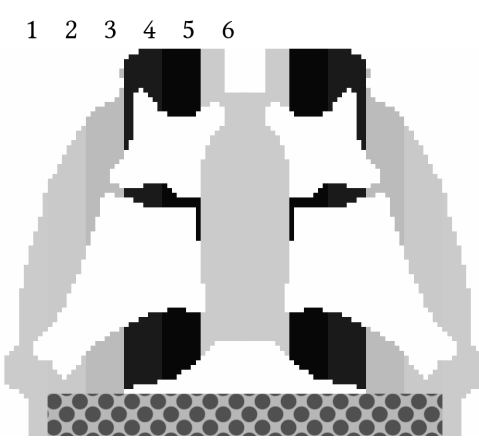

(b) Topologia pós-processada e gradação do caso de camadas perpendiculares;

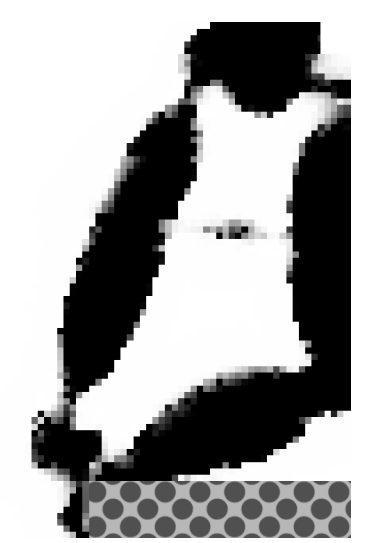

(c) Topologia do caso de camadas paralelas;

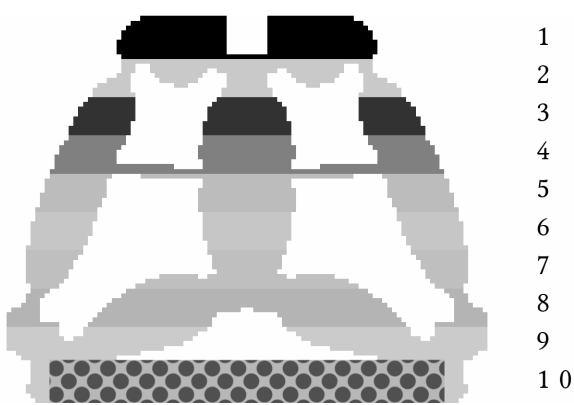

(d) Topologia pós-processada e gradação do caso de camadas paralelas.

Figura 5.11: Garras, sujeitas a restrições, com gradação funcional Cobre-Níquel restrita em camadas.

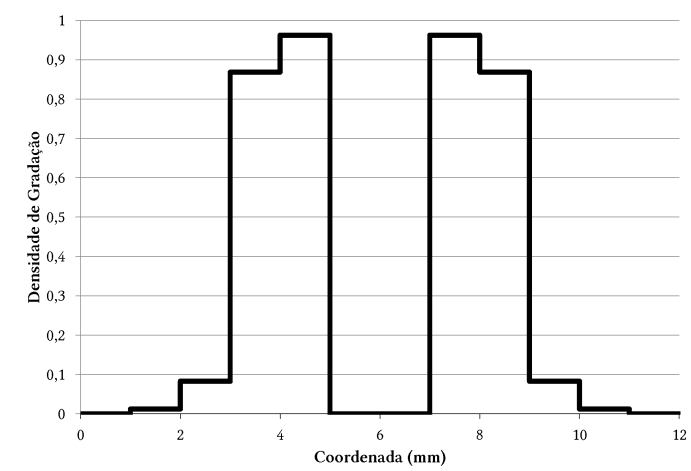

(a) Gradação em camadas perpendiculares à cerâmica;

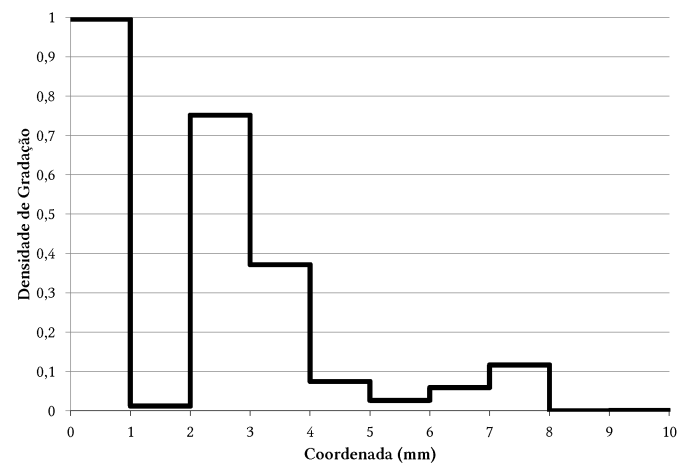

(b) Gradação em camadas paralelas à cerâmica.

Figura 5.12: Distribuição da gradação nas garras otimizadas.

\subsubsection{Otimização sem Restrições de Volume e Manufatura}

Nesta Seção, o problema de otimização enunciado pela Equação (3.4) é implementado sem restrição de volume, ou seja, com $\Theta_{1}=V_{\Omega_{s}}$. Além disso, não são impostas restrições 
Tabela 5.5: Deslocamentos estáticos horizontais médios de $\Gamma_{t}$ nas garras otimizadas sujeitas a restrições.

\begin{tabular}{ccc}
\hline Figura & Resultado do MOT $(\mathrm{nm})$ & Pós-Processado $(\mathrm{nm})$ \\
\hline $5.2(\mathrm{a})$ - Cobre & 5,31 & 5,40 \\
$5.2(\mathrm{c})$ - Níquel & 7,65 & 7,64 \\
$5.3(\mathrm{a})$ - MGF & 9,68 & 10,31 \\
$5.3(\mathrm{c})$ - MGF & 9,46 & 9,88 \\
\hline
\end{tabular}

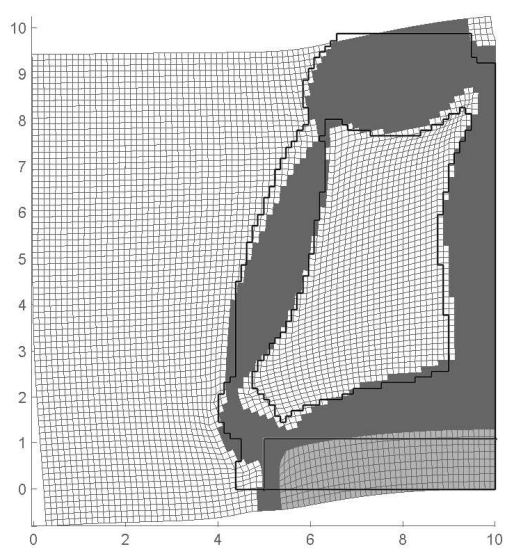

(a) Caso de Cobre homogêneo;

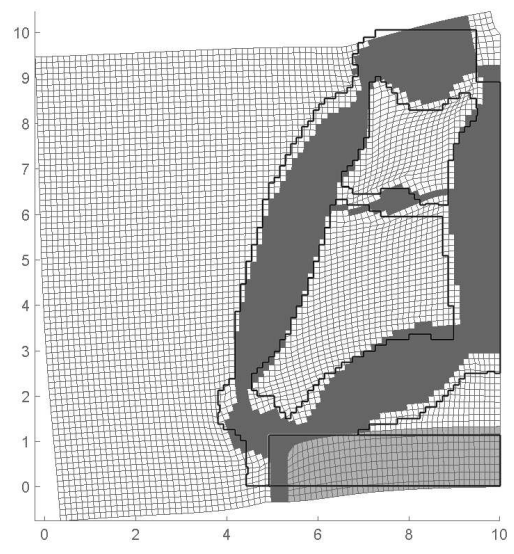

(c) Caso de camadas perpendiculares;

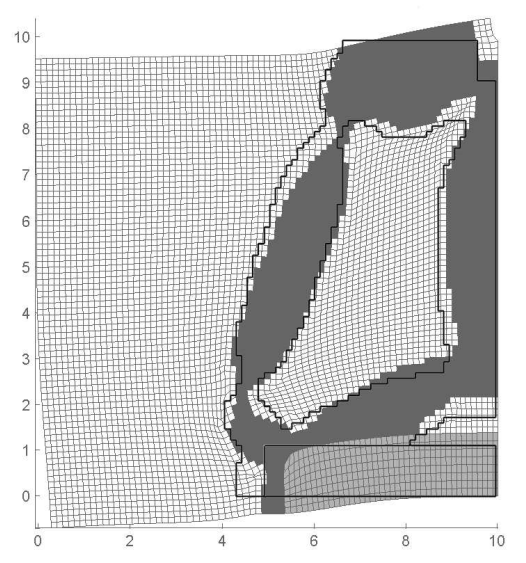

(b) Caso de Níquel homogêneo;

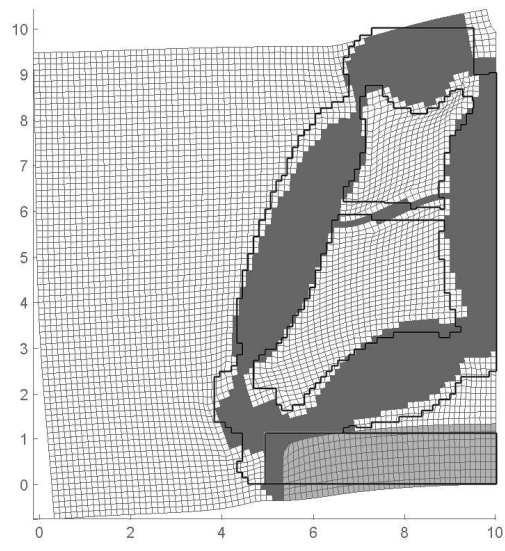

(d) Caso de camadas paralelas.

Figura 5.13: Deformadas das garras otimizadas sujeitas a restrições.

de gradação em camadas, permitindo que o material seja distribuído livremente dentro da estrutura. Isto é feito para avaliar a influência das restrições impostas na Seção anterior no resultado final obtido. Neste problema, as variáveis de topologia $\left(\rho_{1}\right)$ são inicializadas com valor de 0,5 e as de gradação $\left(\rho_{2}\right)$ com valor de 0,5 . 
O resultado obtido através do MOT possui regiões cinzas, conforme evidenciam as Figuras 5.14(a) e 5.14(c), que apresentam as topologias obtidas para os casos de Cobre e Níquel homogêneos. Essas topologias necessitam de um pós-processamento que estabelece uma densidade de corte para a definição da presença de material - adotada como igual a 0,7 nestes casos. Assim, os formatos finais das garras, considerando a simetria, são apresentados nas Figuras 5.14(b) e 5.14(d).

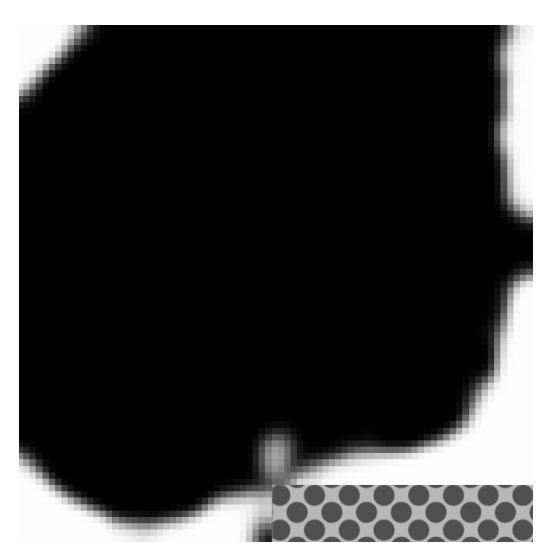

(a) Topologia do caso de Cobre;

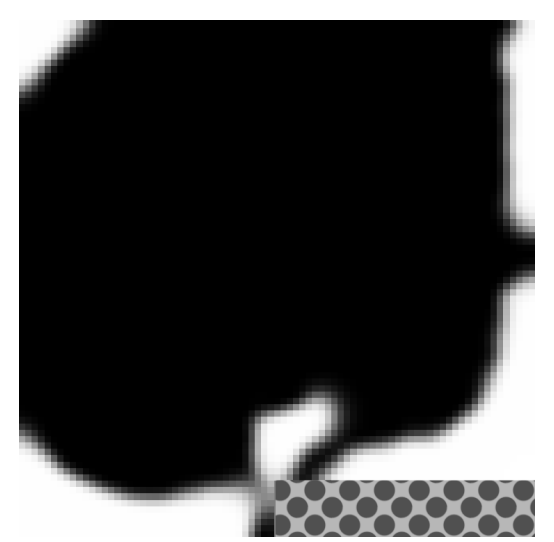

(c) Topologia do caso de Níquel;

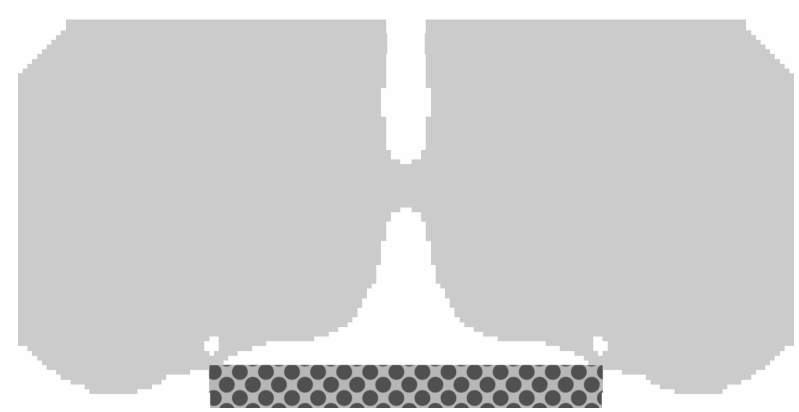

(b) Topologia pós-processada do caso de Cobre;

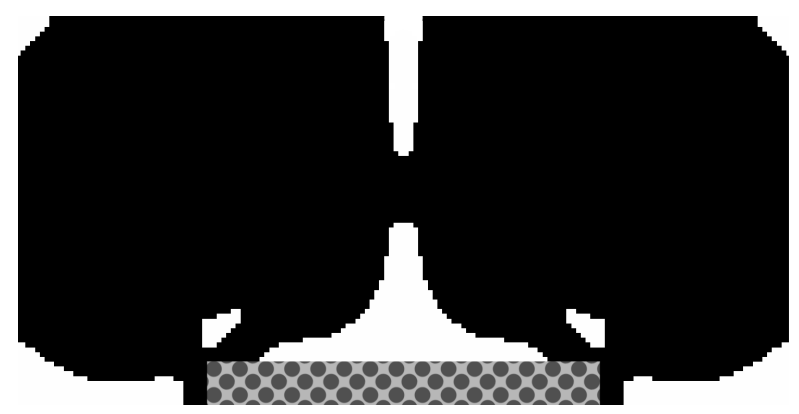

(d) Topologia pós-processada do caso de Níquel.

Figura 5.14: Garras com estrutura acoplada homogênea.

Ao incluir a gradação livre de Cobre-Níquel no domínio otimizável, é obtida a topologia da Figura 5.15(a), na qual é utilizada projeção, dada pela Equação (2.6), para as variáveis de gradação $\left(\rho_{2}\right)$ com grau $g=1$ e $R_{\max }$ equivalente ao lado de 12 elementos. Após o processamento deste resultado (com densidade de corte igual a 0,7 ), chega-se à forma final da garra, apresentada na Figura 5.15(b), onde as regiões mais claras se aproximam das propriedades elásticas do Cobre e as mais escuras das do Níquel.

Os deslocamentos estáticos horizontais médios dos nós pertencentes à região $\Gamma_{t}$ das garras, devido à aplicação $-100 \mathrm{~V}$ ao eletrodo livre da cerâmica, são apresentados na Tabela 5.6. Esses deslocamentos são ilustrados na Figura 5.16, que apresenta a deformada de cada caso das garras otimizadas. 


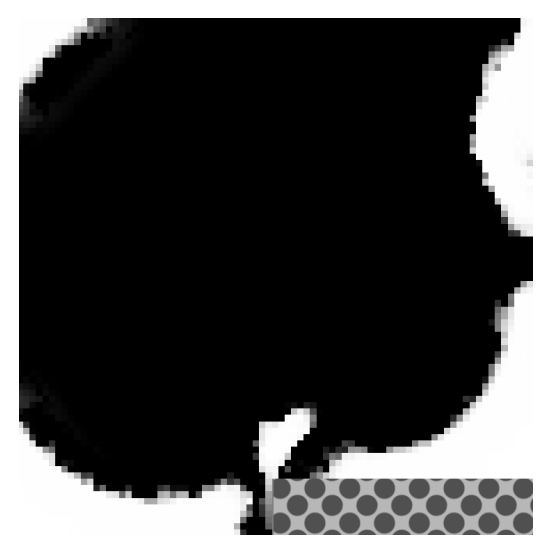

(a) Topologia do caso gradado;

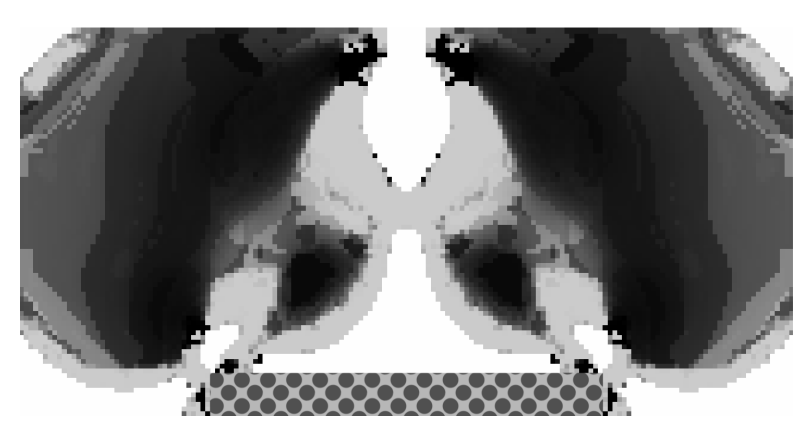

(b) Topologia pós-processada e gradação do caso gradado;

Figura 5.15: Garra com gradação funcional livre de Cobre-Níquel.

Tabela 5.6: Deslocamentos estáticos horizontais médios de $\Gamma_{t}$ nas garras otimizadas.

\begin{tabular}{ccc}
\hline Figura & Resultado do MOT $(\mathrm{nm})$ & Pós-Processado $(\mathrm{nm})$ \\
\hline $5.14(\mathrm{a})$ - Cobre & 6,65 & 7,00 \\
$5.14(\mathrm{c})$ - Níquel & 10,34 & 10,96 \\
$5.15(\mathrm{a})$ - MGF & 11,79 & 12,09 \\
\hline
\end{tabular}

\subsubsection{Discussão}

Em relação ao objetivo do projeto, os resultados sem restrições de volume e manufatura (Seção 5.2.2) mostram que ao utilizar a gradação funcional Cobre-Níquel o deslocamento de saída é 15,6\% superior ao melhor caso homogêneo (Níquel), como evidenciado na Tabela 5.6.

Quando se impõem restrições (Seção 5.2.1), as estruturas gradadas apresentam resultados superiores aos dos casos homogêneos. Entre os homogêneos, como esperado (ver Seção 5.1.3), o maior deslocamento é observado na estrutura em Níquel $(7,64 \mathrm{~nm})$, enquanto nas estruturas gradadas são observados deslocamentos de $9,88 \mathrm{~nm}$ na estrutura com gradação paralela à cerâmica e de 10,31nm na com gradação perpendicular - Tabela 5.5. Ou seja, a gradação levou a resultados até 34,90\% superiores aos de estruturas homogêneas.

\subsection{Sensor de Força Piezelétrico}

Nesta Seção são apresentados os resultados da otimização de um sensor de força piezelétrico, onde o objetivo é maximizar a diferença de potencial entre os eletrodos da cerâmica $\Omega_{p}$, baseando-se no domínio de projeto da Figura 5.17. Na representação, $\Omega_{s}$ é 


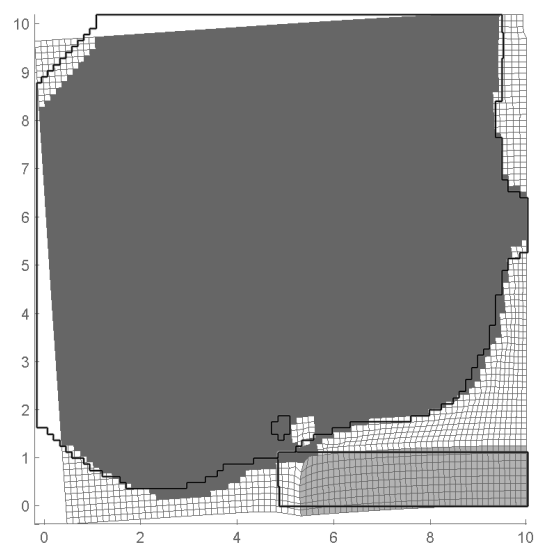

(a) Caso de Cobre homogêneo;

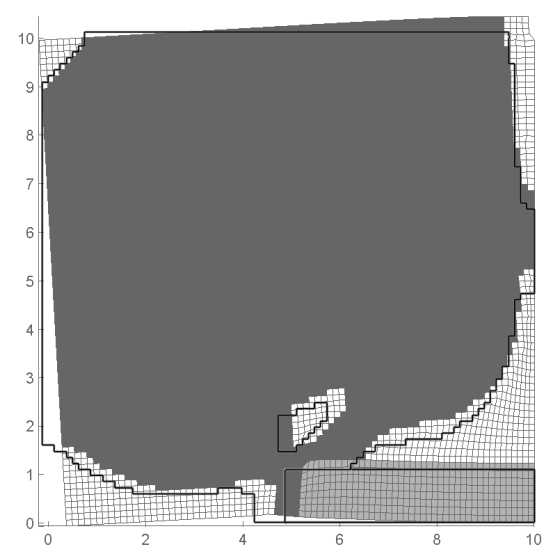

(b) Caso de Níquel homogêneo;

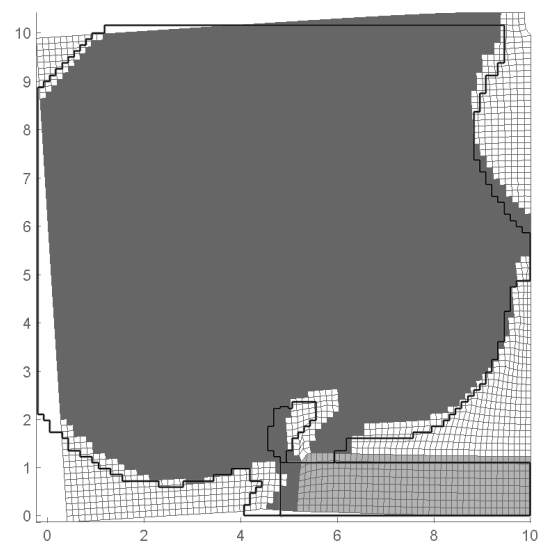

(c) Caso de gradação Cobre-Níquel.

Figura 5.16: Deformadas das garras otimizadas.

o domínio metálico otimizável e $\Gamma_{t}$ denota a região de aplicação da força a ser medida. O domínio do problema apresentado é discretizado em uma malha de $80 \times 40$ elementos para proceder com a otimização enunciada na Equação (3.20). Considera-se $\Omega_{s}$ constituída por Cobre e Níquel, cujas propriedades elásticas são obtidas experimentalmente e apresentadas na Tabela 6.2. Em $\Omega_{p}$, são consideradas as propriedades do PZT-5A do Anexo B.

O valor de rigidez da mola $k_{\text {mola }}$ é estabelecido em $50 \mathrm{~N} / \mathrm{mm}$ e se adota $\mathbf{d}_{1}^{\text {min }}=10^{-6}$. Para as variáveis topologia, o coeficiente de penalização é variado de 1 a 7 , seguindo o MC, e é utilizada a projeção dada pela Equação (2.6) com grau $g=2$ e $R_{\max }$ equivalente ao lado de 4 elementos. 

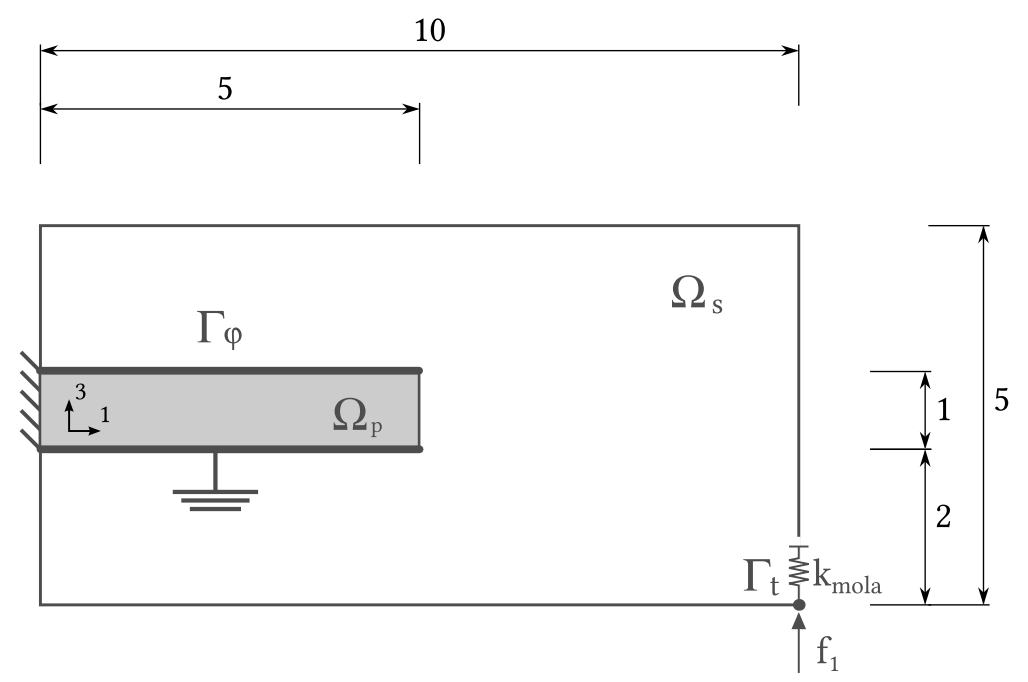

Figura 5.17: Domínio de projeto de sensor de força piezelétrico (dimensões em $\mathrm{mm}$ ).

\subsubsection{Otimização com Restrições de Volume e Manufatura}

Nesta Seção, o problema de otimização enunciado pela Equação (3.4) é implementado com restrição de volume $\Theta_{1}=0,4 V_{\Omega_{s}}$ e restrição da gradação em camadas com espessura de $1 \mathrm{~mm}$, visando a manufaturabilidade. Porém, com a imposição desta restrição de manufatura, surge um problema de condicionamento numérico que é contornado da forma detalhada na Seção 5.1.1. Neste problema, as variáveis de topologia $\left(\rho_{1}\right)$ são inicializadas com valor de 0,2 e as de gradação $\left(\rho_{2}\right)$ com valor de 0,5 .

O resultado obtido através do MOT possui regiões cinzas, conforme evidencia a Figura 5.18, que apresentam as topologias obtidas para os casos de Cobre e Níquel homogêneos. Essas topologias necessitam de um pós-processamento que estabelece uma densidade de corte para a definição da presença de material - adotada como igual a 0,7 nestes casos. Assim, os formatos finais dos sensores são apresentados nas Figuras 5.18(b) e 5.18(d).

As Figuras 5.19(a) e 5.19(c) apresentam os resultados para os casos com gradação em camadas perpendiculares e paralelas à cerâmica, cujas pseudodensidades são obtidas pelo MOT. Após o processamento dos resultados (com densidade de corte igual a 0,7), são obtidas as formas finais das garras apresentadas nas Figuras 5.19(b) e 5.19(d), onde as camadas mais claras se aproximam das propriedades elásticas do Cobre e as mais escuras das do Níquel. As pseudodensidades de gradação $\rho_{2}$ são dadas na Tabela 5.7 e representadas graficamente na Figura 5.20.

Os potenciais elétricos observados no eletrodo $\Gamma_{\varphi}$ dos sensores, devido à aplicação de uma força unitária em $\Gamma_{t}$, são dados na Tabela 5.8 e os deslocamentos da face de sensoriamento são apresentados na Tabela 5.9. Esses potenciais são gerados pela deformação ilustrada na Figura 5.21. 


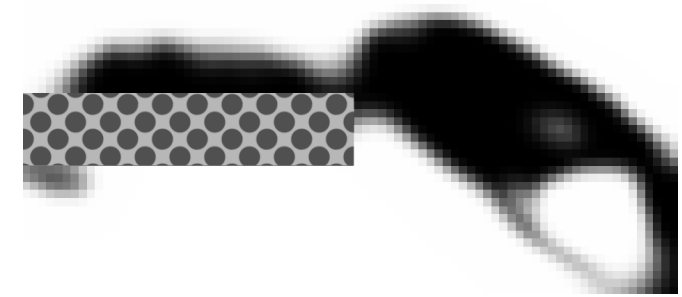

(a) Topologia do caso de Cobre;

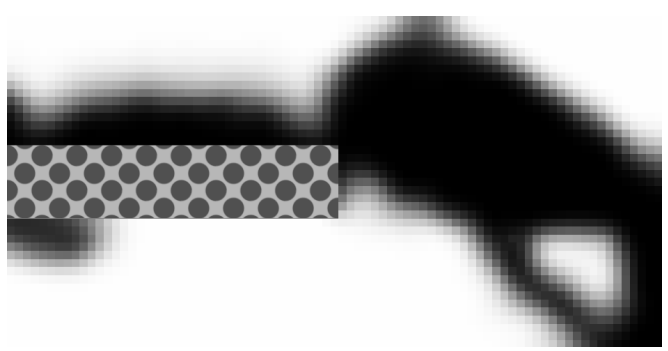

(c) Topologia do caso de Níquel;

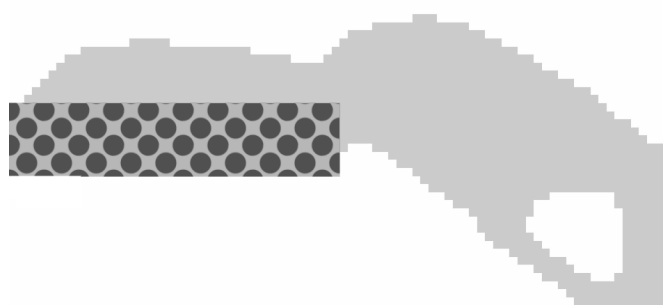

(b) Topologia pós-processada do caso de Cobre;

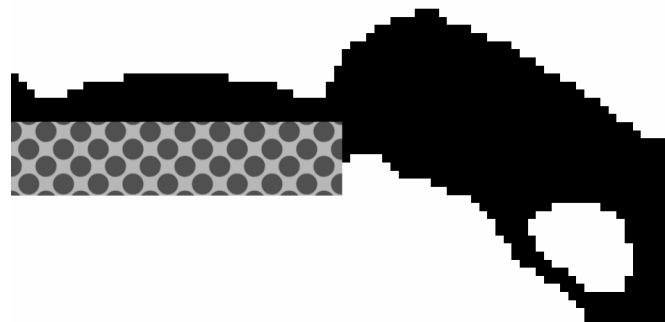

(d) Topologia pós-processada do caso de Níquel.

Figura 5.18: Sensores, sujeitos a restrições, com estrutura acoplada homogênea.

Tabela 5.7: Pseudodensidades de gradação dos sensores otimizados sujeitos a restrições.

\begin{tabular}{ccc}
\hline Camada & $5.19(\mathrm{~b})$ & (b) $5.19(\mathrm{~d})$ \\
\hline 1 & 0,0000 & 0,9999 \\
2 & 0,0000 & 1,0000 \\
3 & 0,0000 & 0,0037 \\
4 & 0,0001 & 0,0000 \\
5 & 0,0001 & 0,0327 \\
6 & 0,0010 & \\
7 & 0,9995 & \\
8 & 0,9398 & \\
9 & 0,2208 & \\
10 & 0,9997 & \\
\hline
\end{tabular}

\subsubsection{Otimização sem Restrições de Volume e Manufatura}

Nesta Seção, o problema de otimização enunciado pela Equação (3.20) é implementado sem restrição de volume, ou seja, com $\Theta_{1}=V_{\Omega_{s}}$. Além disso, não são impostas restrições 


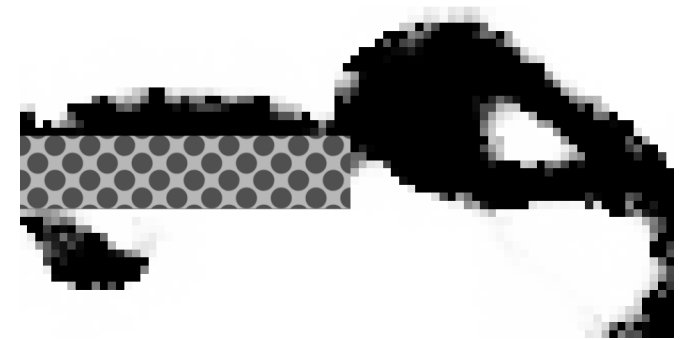

(a) Topologia do caso de camadas perpendiculares;

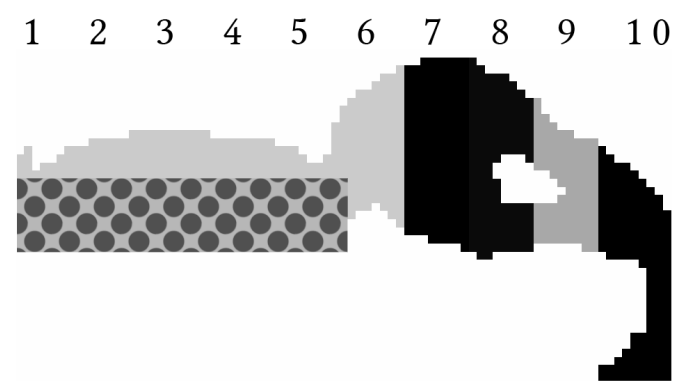

(b) Topologia pós-processada e gradação do caso de camadas perpendiculares;

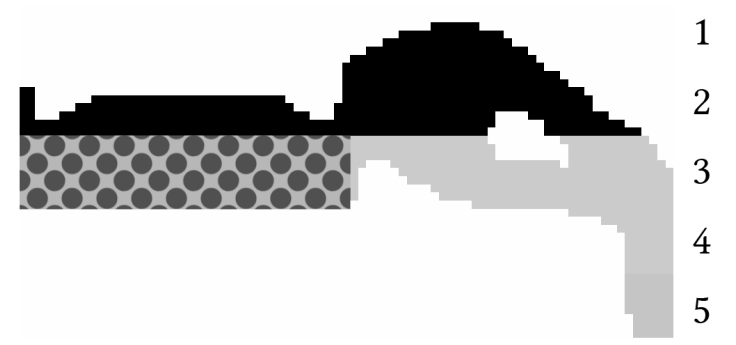

(d) Topologia pós-processada e gradação do caso de camadas paralelas.

Figura 5.19: Sensores, sujeitos a restrições, com gradação funcional Cobre-Níquel restrita em camadas.

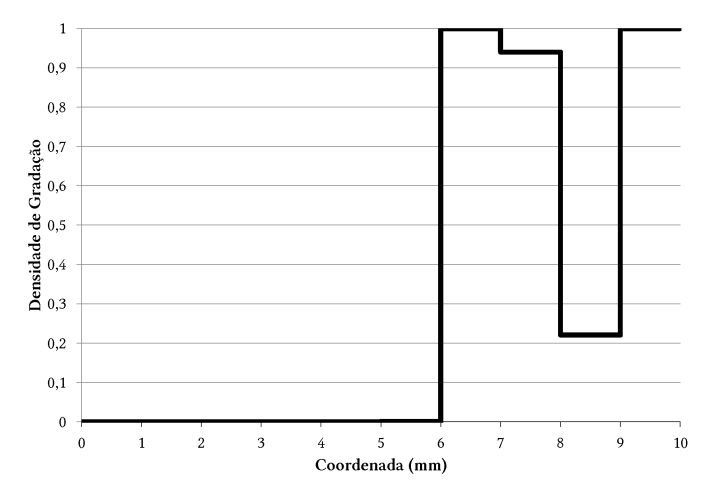

(a) Gradação em camadas perpendiculares à (b) Gradação em camadas paralelas à cerâmica. cerâmica;

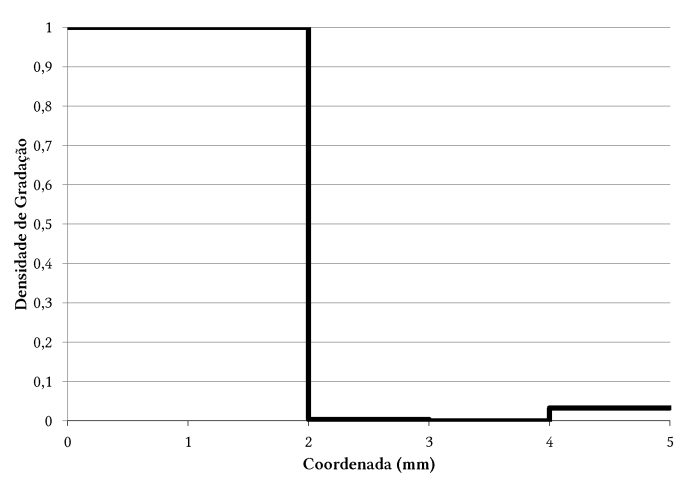

Figura 5.20: Distribuição da gradação nos sensores otimizados.

de gradação em camadas, permitindo que o material seja distribuído livremente dentro da estrutura. Isto é feito para avaliar a influência das restrições impostas na Seção anterior no 
Tabela 5.8: Potenciais elétricos no eletrodo $\Gamma_{\varphi}$ dos sensores otimizados sujeitos a restrições.

\begin{tabular}{ccc}
\hline Figura & Resultado do MOT $(V)$ & Pós-Processado $(V)$ \\
\hline $5.18(\mathrm{a})$ - Cobre & $-22,33$ & $-24,15$ \\
$5.18(\mathrm{c})$ - Níquel & $-33,76$ & $-35,18$ \\
$5.19(\mathrm{a})$ - MGF & $-35,45$ & $-36,62$ \\
$5.19(\mathrm{c})-\mathrm{MGF}$ & $-35,94$ & $-37,02$ \\
\hline
\end{tabular}

Tabela 5.9: Deslocamentos verticais de $\Gamma_{t}$ nos sensores otimizados sujeitos a restrições.

\begin{tabular}{ccc}
\hline Figura & Resultado do MOT $(\mu m)$ & Pós-Processado $(\mu m)$ \\
\hline $5.18($ a $)$ - Cobre & 8,58 & 7,64 \\
$5.18(\mathrm{c})$ - Níquel & 7,09 & 6,40 \\
$5.19(\mathrm{a})$ - MGF & 6,88 & 6,87 \\
$5.19(\mathrm{c})$ - MGF & 6,97 & 7,06 \\
\hline
\end{tabular}

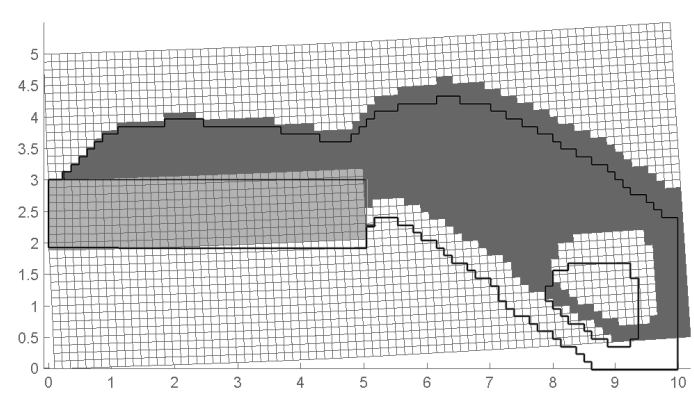

(a) Caso de Cobre homogêneo ;

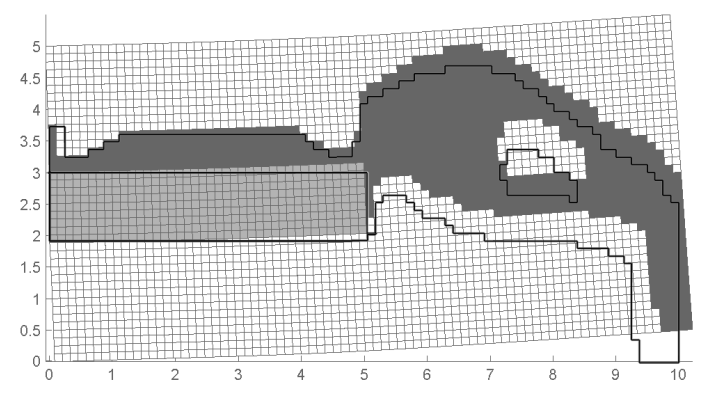

(c) Caso de camadas perpendiculares;

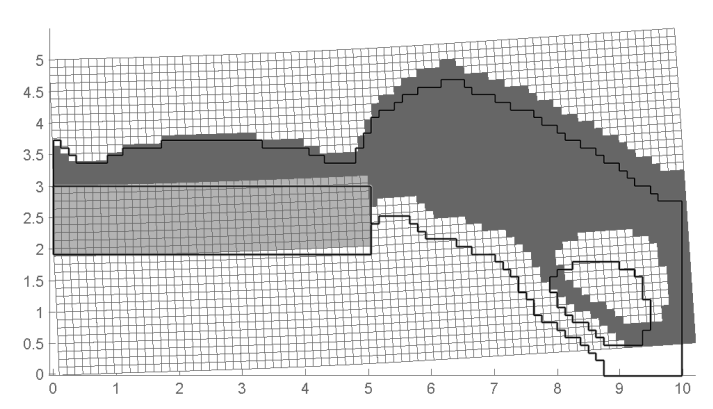

(b) Caso de Níquel homogêneo;

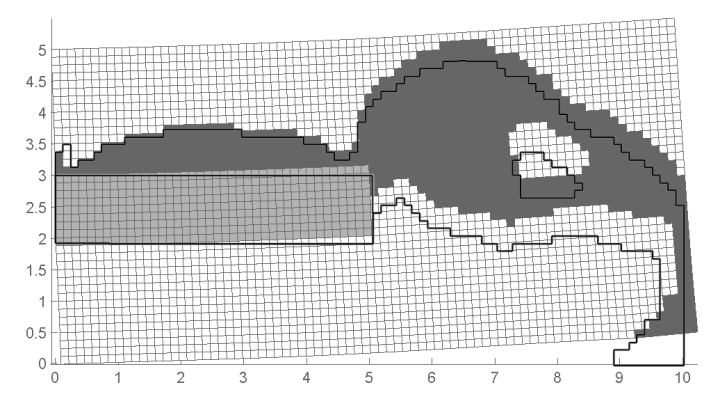

(d) Caso de camadas paralelas.

Figura 5.21: Deformadas dos sensores otimizados sujeitos a restrições.

resultado final obtido. Neste problema, as variáveis de topologia $\left(\rho_{1}\right)$ são inicializadas com 
valor de 0,5 e as de gradação $\left(\rho_{2}\right)$ com valor de 0,5 .

O resultado obtido através do MOT possui regiões cinzas, conforme evidenciam as Figuras 5.22(a) e 5.22(c), que apresentam as topologias obtidas para os casos de Cobre e Níquel homogêneos. Essas topologias necessitam de um pós-processamento que estabelece uma densidade de corte para a definição da presença de material - adotada como igual a 0,7 nestes casos. Assim, os formatos finais dos sensores são apresentados nas Figuras 5.22(b) e $5.22(\mathrm{~d})$.

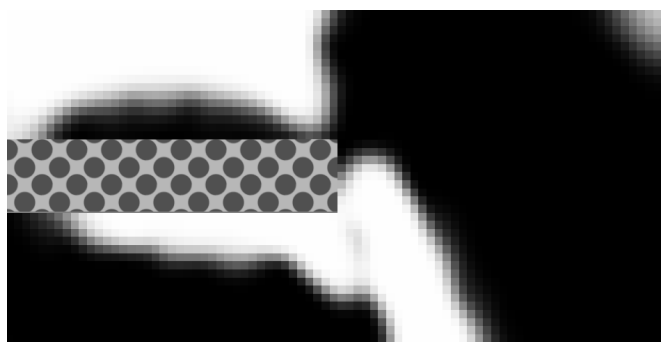

(a) Topologia do caso de Cobre;

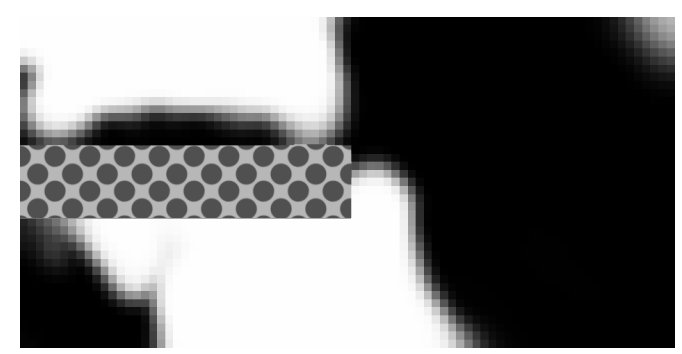

(c) Topologia do caso de Níquel;

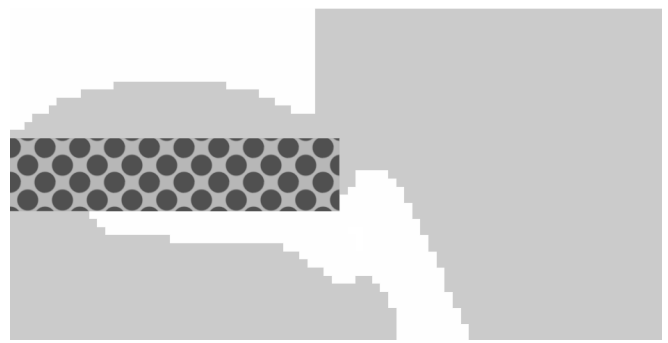

(b) Topologia pós-processada do caso de Cobre;

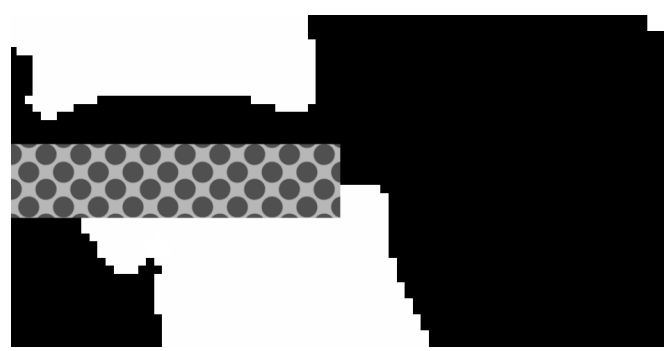

(d) Topologia pós-processada do caso de Níquel.

Figura 5.22: Sensores com estrutura acoplada homogênea.

Ao incluir a gradação livre de Cobre-Níquel no domínio otimizável, é obtida a topologia da Figura 5.23(a), na qual é utilizada projeção, dada pela Equação (2.6), para as variáveis de gradação $\left(\rho_{2}\right)$ com grau $g=1$ e $R_{\max }$ equivalente ao lado de 12 elementos. Após o processamento deste resultado (com densidade de corte igual a 0,7), chega-se à forma final do sensor, apresentada na Figura 5.23(b), onde as regiões mais claras se aproximam das propriedades elásticas do Cobre e as mais escuras das do Níquel.

Os potenciais elétricos observados no eletrodo $\Gamma_{\varphi}$ dos sensores, devido à aplicação de uma força unitária em $\Gamma_{t}$, são dados na Tabela 5.10 e os deslocamentos da face de sensoriamento são apresentados na Tabela 5.9. Esses potenciais são gerados pela deformação ilustrada na 


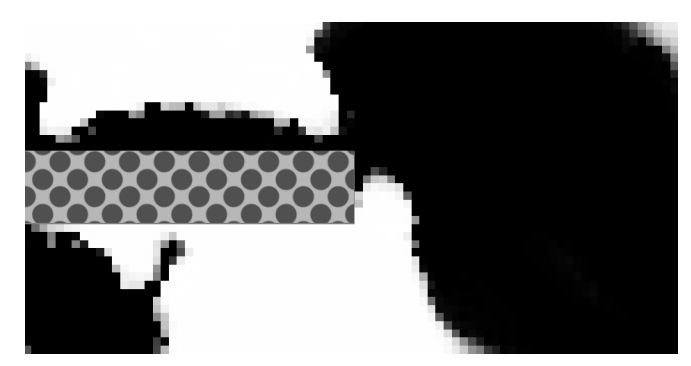

(a) Topologia do caso gradado;

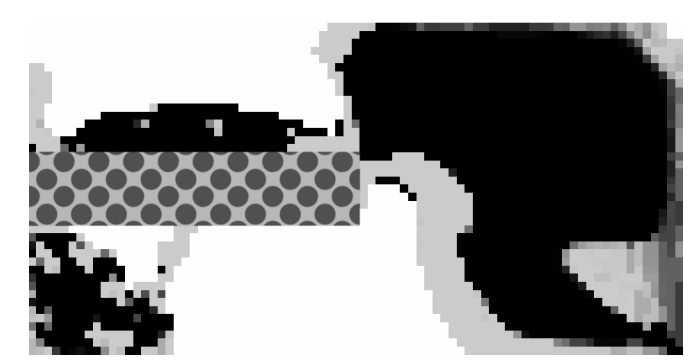

(b) Topologia pós-processada e gradação do caso gradado;

Figura 5.23: Sensor com gradação funcional livre de Cobre-Níquel.

Figura 5.24.

Tabela 5.10: Potenciais elétricos no eletrodo $\Gamma_{\varphi}$ dos sensores otimizados.

\begin{tabular}{ccc}
\hline Figura & Resultado do MOT $(V)$ & Pós-Processado $(V)$ \\
\hline $5.22(\mathrm{a})$ - Cobre & 22,98 & 27,01 \\
$5.22(\mathrm{c})$ - Níquel & 33,53 & 33,77 \\
$5.23(\mathrm{a})$ - MGF & 36,87 & 37,36 \\
\hline
\end{tabular}

Tabela 5.11: Deslocamentos verticais de $\Gamma_{t}$ nos sensores otimizados.

\begin{tabular}{ccc}
\hline Figura & Resultado do MOT $(\mu m)$ & Pós-Processado $(\mu m)$ \\
\hline $5.22(\mathrm{a})$ - Cobre & 8,09 & 7,48 \\
$5.22(\mathrm{c})$ - Níquel & 6,69 & 5,54 \\
$5.23(\mathrm{a})$ - MGF & 6,32 & 6,28 \\
\hline
\end{tabular}

\subsubsection{Discussão}

Em relação ao objetivo do projeto, os resultados sem restrições de volume e manufatura (Seção 5.3.2) mostram que ao utilizar a gradação funcional Cobre-Níquel a diferença de potencial entre os eletrodos da cerâmica é 10,6\% superior ao melhor caso homogêneo (Níquel), como evidenciado na Tabela 5.10.

Porém, ao não impor uma restrição de volume, nota-se a formação de regiões pouco relevantes para o objetivo de projeto, como a concentração de material abaixo da cerâmica - Figuras 5.22 e 5.23 . 


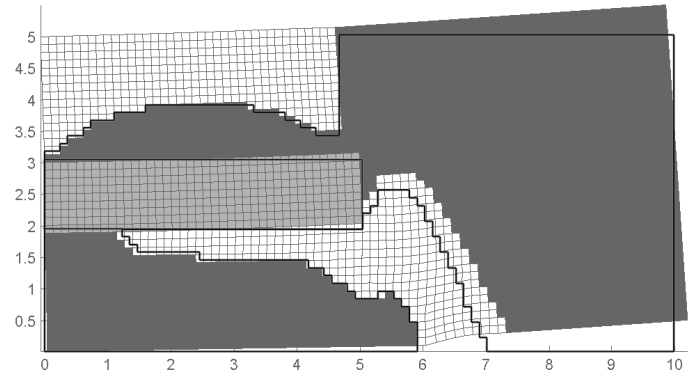

(a) Caso de Cobre homogêneo ;

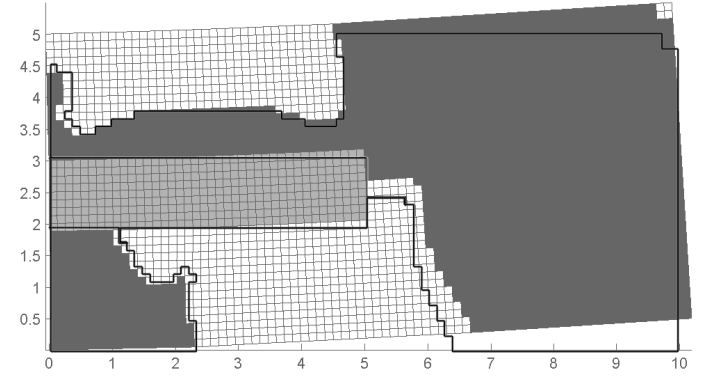

(b) Caso de Níquel homogêneo;

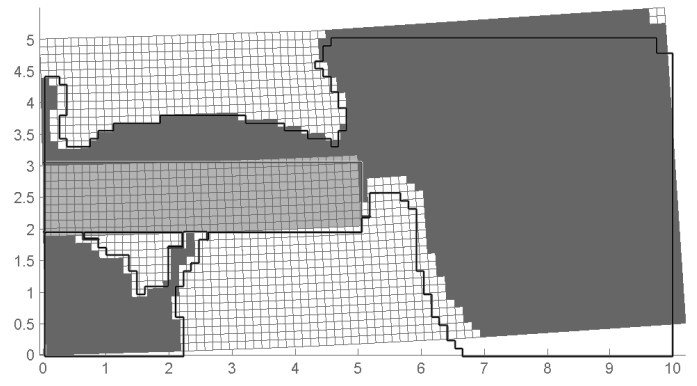

(c) Caso de gradação Cobre-Níquel;

Figura 5.24: Deformadas dos sensores otimizados.

Ao impor restrições (Seção 5.3.1), as estruturas gradadas apresentam resultados ligeiramente superiores aos dos casos homogêneos. Entre os casos homogêneos, a maior diferença de potencial entre os eletrodos da cerâmica é observada na estrutura constituída por Níquel: $35,18 \mathrm{~V}$. Já entre os gradados, as diferenças de potencial são de 37,02 $\mathrm{V}$ na estrutura com gradação paralela à cerâmica e de $36,63 \mathrm{~V}$ na com gradação perpendicular. Estes valores representam um aumento de 5,24\% e 4,13\%, respectivamente, em relação ao melhor caso homogêneo.

Nota-se que, dentre os homogêneos, os casos de Níquel sempre gera uma maior diferença de potencial elétrico entre os eletrodos da cerâmica do que os casos de Cobre. Apesar disto, os deslocamentos dos pontos de sensoriamento dos casos de Níquel são sempre inferiores aos dos pontos dos casos de Cobre. Isto é esperado e condizente com o discutido na Seção 5.1.3, já que o Níquel, por ser mais rígido, transmite melhor os deslocamentos da face de sensoriamento até os eletrodos das cerâmicas. Já o Cobre, que é mais flexível, mitiga boa parte dos deslocamentos da face de sensoriamento através de sua própria deformação estrutural.

Também se nota que praticamente todas as camadas possuem propriedades muito 
próximas do Cobre e do Níquel puro, ou seja, $\rho_{2} \simeq 0$ e $\rho_{2} \simeq 1$. Isto é indicativo de que, para o problema do sensor, materiais intermediários não implicam em grandes vantagens no objetivo de projeto. 


\section{Resultados Experimentais e Discussões}

Este Capítulo apresenta os resultados de procedimentos experimentais para a caracterização de materiais sinterizados e para a fabricação e verificação de um protótipo de dispositivo piezelétrico gradado.

\subsection{Protótipos com Formas Arbitrárias}

Visando verificar a execução do processo de fabricação proposto para a fabricação de dispositivos funcionalmente gradados, são fabricados protótipos com formatos arbitrários.

\subsubsection{Fabricação}

Para os primeiros protótipos, a fabricação se origina na sinterização de uma peça com $20 \mathrm{~mm}$ de diâmetro e $3 \mathrm{~mm}$ de altura, sendo composta por três camadas de $1 \mathrm{~mm}$ constituídas, na ordem, por: Níquel puro, mistura aproximadamente homogênea Cobre-Níquel (50 - 50 em massa) e Cobre puro. O parâmetros do processo de SJP são baseados em Rubio (2010), cujas curvas de temperatura e pressão mecânica são apresentadas na Figura 6.1. Em seguida, a peça gradada é fatiada por eletroerosão como ilustra a Figura 6.2.

As fatias gradadas são usinadas por corte a laser, obtendo-se o formato final desejado para as estruturas metálicas acopladas dos protótipos. Essas estruturas são coladas, via epóxi rápido, a cerâmicas piezoelétricas, cujos formatos finais são obtidos pela utilização de uma serra de precisão Disco DAD-322 com disco diamantado ZH05-SD1500-N1-50II (DISCO CORPORATION, 2012). A montagem final de dois protótipos com topologias arbitrárias são apresentados na Figura 6.3, onde as regiões mais próximas às cerâmicas são constituídas por Cobre e as mais distantes por Níquel. 


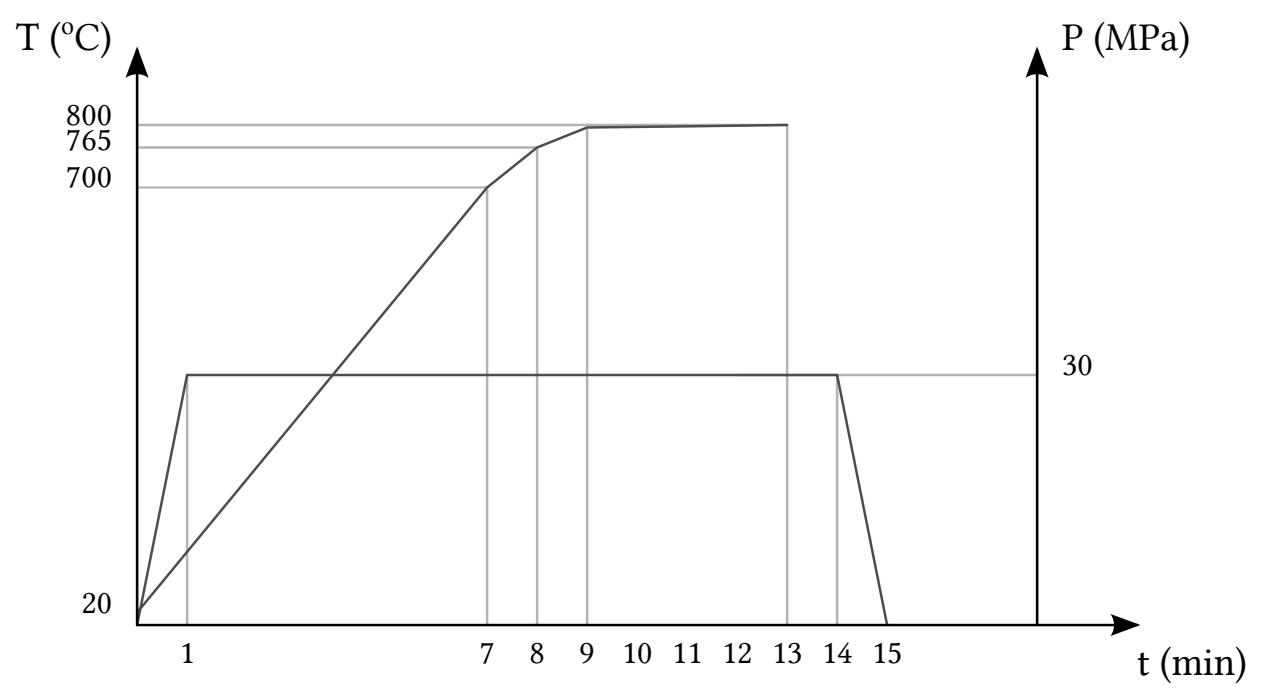

Figura 6.1: Curvas de temperatura e pressão mecânica da sinterização de amostras em Cobre e Níquel.

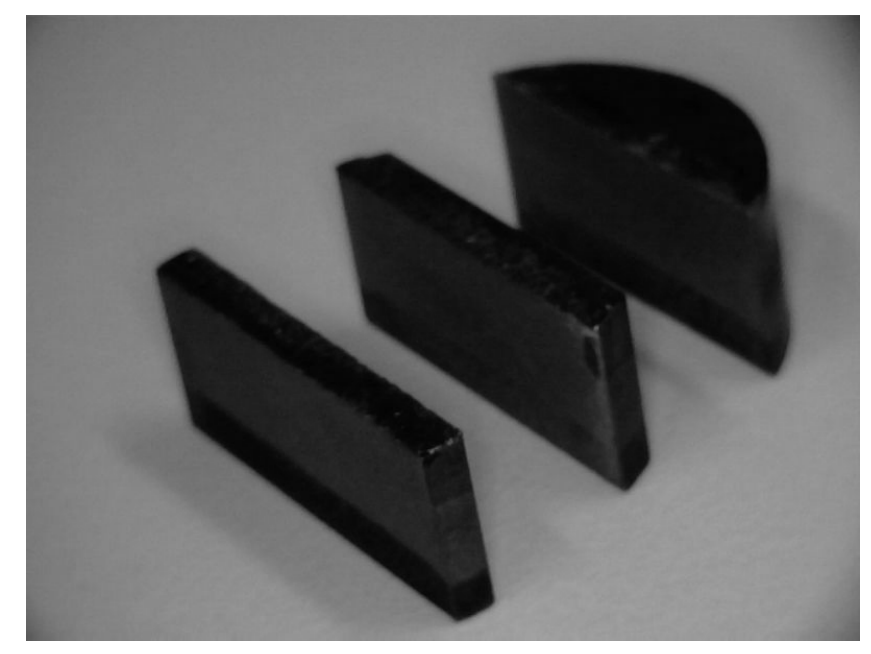

Figura 6.2: Eletroerosão de peça com gradação arbitrária.

\subsubsection{Discussão}

Pela fabricação de protótipos com topologia e gradação arbitrários foi possível verificar a exequibilidade do processo de fabricação proposto. Porém, a colagem das estruturas metálicas às cerâmicas mostrou-se pouco resistente e imprecisa. Para amenizar este gargalo, é interessante testar adesivos específicos para esta finalidade, como o Loctite Tile Bond High Temp Red RTV (HENKELNA, 2012).

\subsection{Gradação Cobre-Níquel}

Tendo em vista o levantamento das propriedades da gradação funcional Cobre-Níquel, realiza-se a sinterização de amostras cilíndricas homogêneas de Cobre e de Níquel que são 


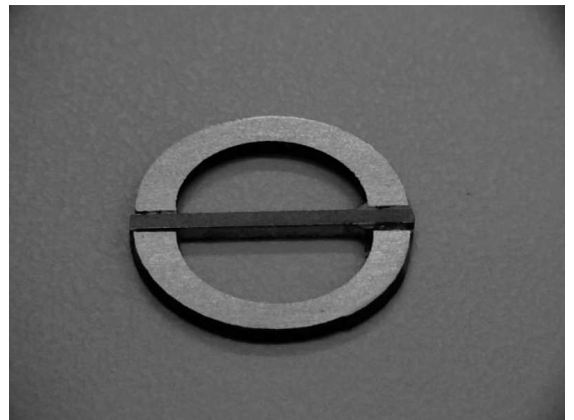

(a) Protótipo arbitrário circular;

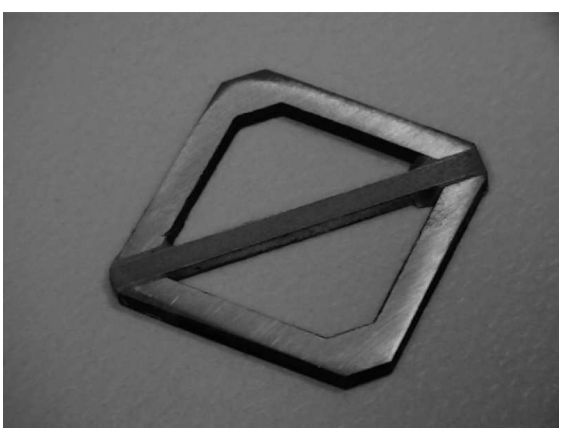

(b) Protótipo arbitrário triangular;

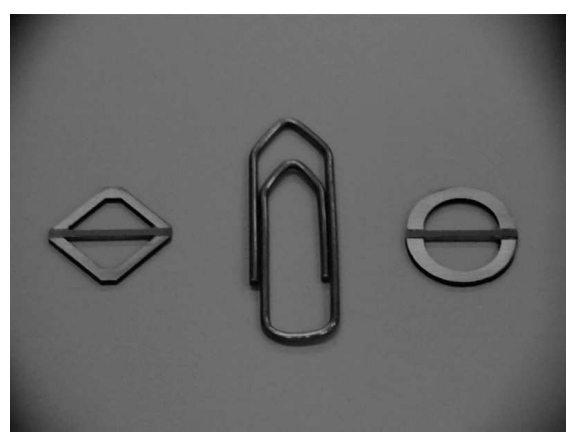

(c) Noção de escala.

Figura 6.3: Protótipos de dispositivos piezelétricos com topologia arbitrária.

submetidas a ensaios ultrassônicos para que os valores de suas propriedades elásticas sejam utilizadas na implementação do MOT, como descrito na Figura 4.1.

\subsubsection{Fabricação}

As amostras são fabricadas por processos de SJP idênticos, cujas curvas de temperatura e pressão mecânica são dadas na Figura 6.1.

Após serem sinterizadas, as amostras são usinadas em uma lapidadora Logitech PM5 (LOGITECH LTD., 2012), utilizando abrasivo baseado em pó de carbeto de silício com granulometria de $8 \mu \mathrm{m}$, de forma a deixar as suas faces planas e paralelas entre si, com desvios de paralelismo inferiores a $30 \mu \mathrm{m}$. As propriedades físicas finais das amostras (Tabela 6.1) são obtidas por um micrômetro e por uma balança de precisão, valendo-se do princípio de Arquimedes.

Tabela 6.1: Características físicas das amostras homogêneas.

\begin{tabular}{ccc}
\hline & Espessura $d(\mathrm{~mm})$ & Densidade $\varrho\left(\mathrm{g} / \mathrm{cm}^{3}\right)$ \\
\hline Níquel & 4,950 & 8,635 \\
Cobre & 4,131 & 7,860 \\
\hline
\end{tabular}




\subsubsection{Caracterização}

Ao proceder com os métodos descrito na Seção 4.2, são obtidas as velocidades de propagação relacionadas na Tabela 6.2. Para isto, foram utilizados no experimento um gerador de função Agilent 33250A e um osciloscópio Agilent DSO3102A (AGILENT TECHNOLOGIES, 2012).

Ressalte-se que, no caso da amostra de Cobre, não foi possível identificar mais do que um pico de intensidade no sinal obtido pelo transdutor receptor, devido à forte atenuação do material. Assim, foi necessário recorrer ao método alternativo descrito na mesma Seção, considerando o tempo $t_{a}$ que as ondas tomam para percorrer a distância entre os transdutores quando não há nenhum corpo entre eles.

Tabela 6.2: Velocidades de propagação e constantes elásticas das amostras homogêneas.

\begin{tabular}{ccccc|cc}
\hline & $C_{l}(\mathrm{~m} / \mathrm{s})$ & $C_{c}(\mathrm{~m} / \mathrm{s})$ & $K(\mathrm{GPa})$ & $G(G P a)$ & $E(G P a)$ & $\nu$ \\
\hline Níquel & 5530 & 2962 & 163,10 & 75,77 & 196,83 & 0,2989 \\
Cobre & 3821 & 1973 & 73,94 & 30,60 & 80,67 & 0,3182 \\
\hline
\end{tabular}

\subsubsection{Discussão}

A amostra de Níquel apresenta propriedades próximas do esperado, mas a amostra de Cobre apresenta um módulo de Young abaixo do esperado. Assim, com o objetivo de compreender melhor a causa desses valores dispares, a amostra é submetida a uma análise microscópica - Figura 6.4 .

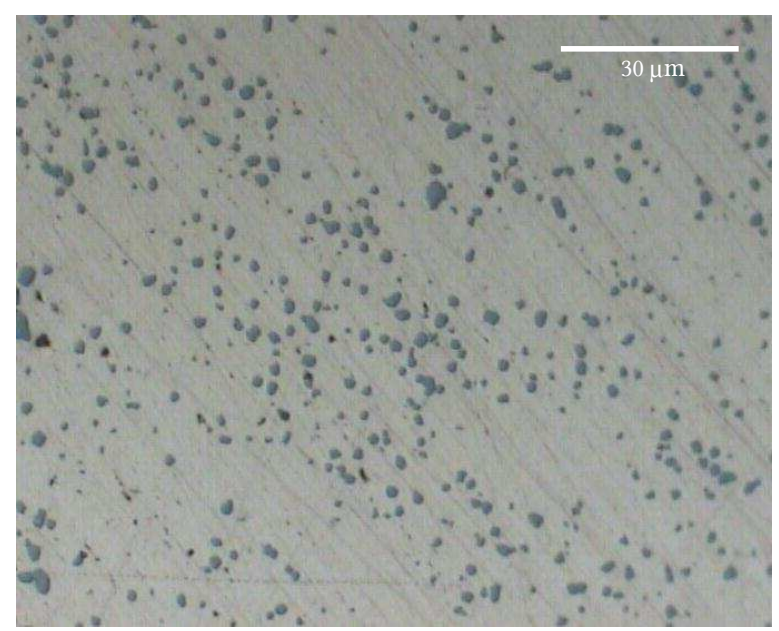

Figura 6.4: Seção da amostra de Cobre vista em microscópio óptico.

Nas imagens é possível notar uma grande quantidade de inclusões de um material mais escuro, praticamente insolúvel no Cobre. As evidências são grandes de que essas inclusões 
sejam compostas por Carbono que migrou do molde de grafite para a amostra, possivelmente afetando as propriedades elásticas da mesma. Através do software Mathematica 8 (WOLFRAM RESEARCH, 2012) foi feita a análise quantitativa do espectro de cores para determinar a área das inclusões escuras na Figura 6.4, determinando-se que esta equivale a 5, 93\% da área total. Considerando as especificações dos fornecedores do molde (MORGANITE BRASIL, 2012) e do pó de Cobre (JB QUíMICA, 2012), os densidades do grafite e do Cobre são, respectivamente, $2,28 \mathrm{~g} / \mathrm{cm}^{3}$ e $8,89 \mathrm{~g} / \mathrm{cm}^{3}$. Assim, estima-se que as inclusões de Carbono representem $1,52 \%$ em massa na amostra.

Essas disparidades também podem ocorrer devido ao método ultrassônico utilizado na caracterização das amostras. É conhecido que ondas sonoras encontram grande dificuldade para se propagar por amostras de cobre sinterizadas, o que é evidenciado pela identificação de apenas um pico de intensidade nas medições. Segundo Page e McCulloch (1986), ocorre uma rápida atenuação conforme os comprimentos de onda se aproximam do comprimento de correlação da percolação do meio, que define a transição entre um sistema de natureza homogênea (Euclidiano) e um sistema fractal. Esse comprimento define a transformação das excitações extensivas na forma de fônons para excitações localizadas na forma de fráctons.

\subsection{Protótipo de Atuador Piezelétrico}

Buscando a verificação dos resultados numéricos obtidos e do processo de fabricação proposto, nesta Seção é detalhada a construção e caracterização de um protótipo do moonie piezelétrico com restrição de gradação $\mathrm{Cu}-\mathrm{Ni}$ em camadas perpendiculares à cerâmica, descrito na Seção 5.1.

\subsubsection{Fabricação}

Como descrito no Capítulo 4, a fabricação de um dispositivo com gradação funcional de material se inicia com a sinterização de um tarugo constituído por camadas com diferentes proporções de materiais. O moonie da Figura 5.3(b) prevê 16 camadas, mas optou-se por desconsiderar as camadas incompletas das extremidades pela dificuldade de controlar espessuras muito finas. Assim, as misturas das 14 camadas restantes são determinadas com base nas curvas $K^{*}$ e $G^{*}$, dadas pelas Equações (4.10) e (4.11).

Considerando o estabelecido nas Equações (4.12) e (4.13), as frações em volume para o caso da gradação Níquel-Cobre são apresentadas na Tabela 6.3. Nela, discriminam-se os valores de rigidez alvo para as pseudodensidades de gradação $\rho_{2}$ e as proporções em volume $v_{N i}^{K}$ e $v_{N i}^{G}$ correspondentes a cada uma destas. Como se nota, estes valores são muito próximos 
e, portanto, é válido utilizar a aproximação de fração em volume média $v_{N i}^{*}$.

Tabela 6.3: Determinação das frações em volume de Níquel a partir da rigidez desejada.

\begin{tabular}{cc|cc|cc|c}
\hline Camada & $\rho_{2}$ & $K_{\text {alvo }}(G P a)$ & $G_{\text {alvo }}(G P a)$ & $v_{N i}^{K}$ & $v_{N i}^{G}$ & $v_{N i}^{*}$ \\
\hline 2 & 0,8430 & 149,10 & 68,68 & 0,8981 & 0,8923 & 0,8952 \\
3 & 0,0152 & 75,29 & 31,29 & 0,0246 & 0,0231 & 0,0238 \\
4 & 0,9485 & 158,51 & 73,44 & 0,9680 & 0,9661 & 0,9670 \\
5 & 0,0355 & 77,10 & 32,20 & 0,0567 & 0,0534 & 0,0550 \\
6 & 0,0020 & 74,11 & 30,69 & 0,0033 & 0,0031 & 0,0032 \\
7 & 0,4749 & 116,28 & 52,05 & 0,5969 & 0,5818 & 0,5894 \\
8 & 0,1357 & 86,04 & 36,73 & 0,2042 & 0,1941 & 0,1991 \\
\hline
\end{tabular}

Sabendo-se a fração em volume de cada componente nas camadas da gradação, basta utilizar as densidades da Tabela 6.1 para prosseguir com a pesagem dos pós de Cobre e Níquel. A Tabela 6.4 apresenta a massa de cada material para um tarugo com $20 \mathrm{~mm}$ de diâmetro com camadas de gradação com $1 \mathrm{~mm}$ de espessura.

Tabela 6.4: Massas de Cobre e Níquel em cada camada.

\begin{tabular}{cc|cc}
\hline Camada & $v_{N i}^{*}$ & $m_{C u}(g)$ & $m_{N i}(g)$ \\
\hline 2 & 0,8952 & 0,2588 & 2,4284 \\
3 & 0,0238 & 2,4105 & 0,0646 \\
4 & 0,9670 & 0,0815 & 2,6231 \\
5 & 0,0550 & 2,3334 & 0,1492 \\
6 & 0,0032 & 2,4613 & 0,0087 \\
7 & 0,5894 & 1,0139 & 1,5988 \\
8 & 0,1991 & 1,9776 & 0,5401 \\
\hline
\end{tabular}

Em seguida, os pós de cada camada são misturados manualmente, afim de obter uma mistura aproximadamente homogênea, e depositados no molde como detalhado na Seção 4.4. A sinterização do tarugo gradado segue a mesma rotina das amostras caracterizadas por ultrassom, apresentada na Figura 6.1.

Com o tarugo sinterizado, procede-se com a eletroerosão e corte a laser, como ilustrado na Figura 6.5. Na sequência, as estruturas gradadas são coladas a uma tira de PZT-5A comercial, dando a forma final ao protótipo - Figura 6.6. Finalmente, para que o protótipo se torne funcional, soldam-se fios aos eletrodos da cerâmica piezelétrica, como mostra a Figura 6.7. 


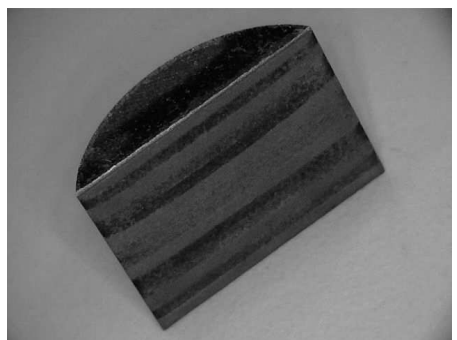

(a) Sinterização;

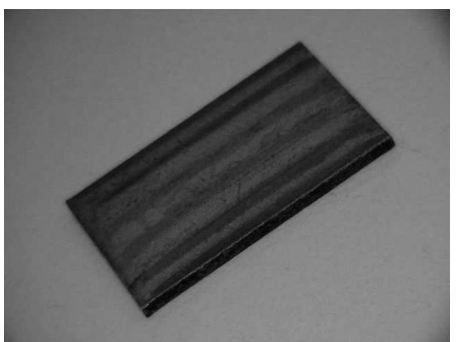

(b) Eletroerosão;

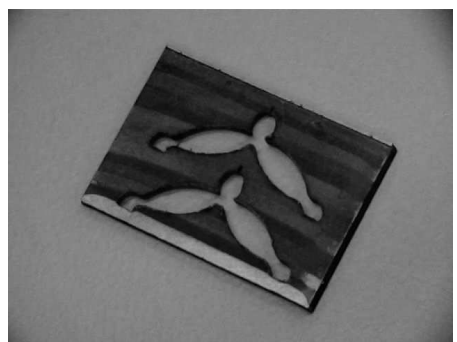

(c) Corte a laser;

Figura 6.5: Peças obtidas em cada etapa do processo de fabricação.

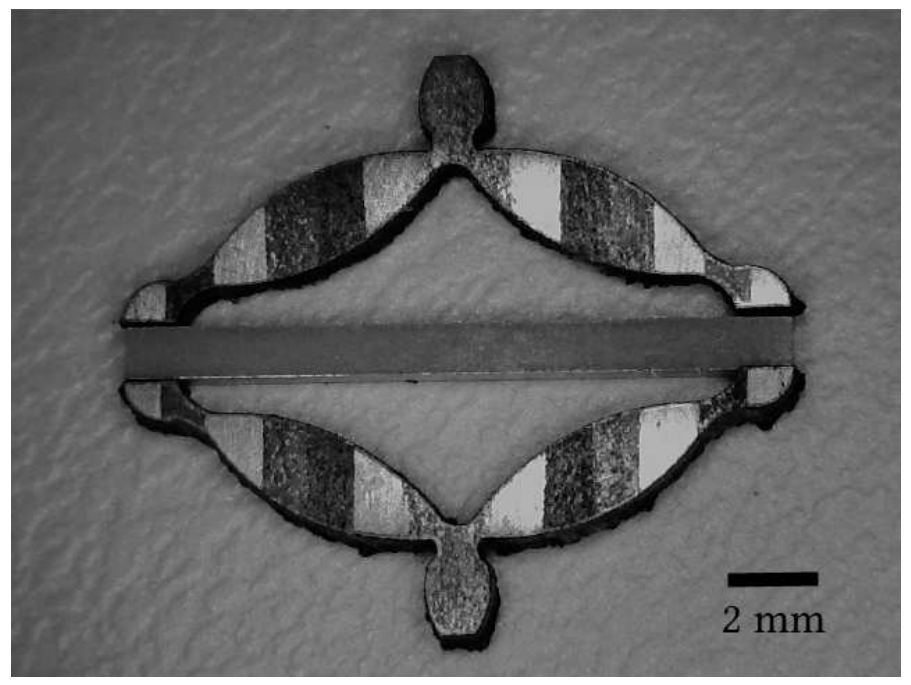

Figura 6.6: Protótipo de atuador gradado.

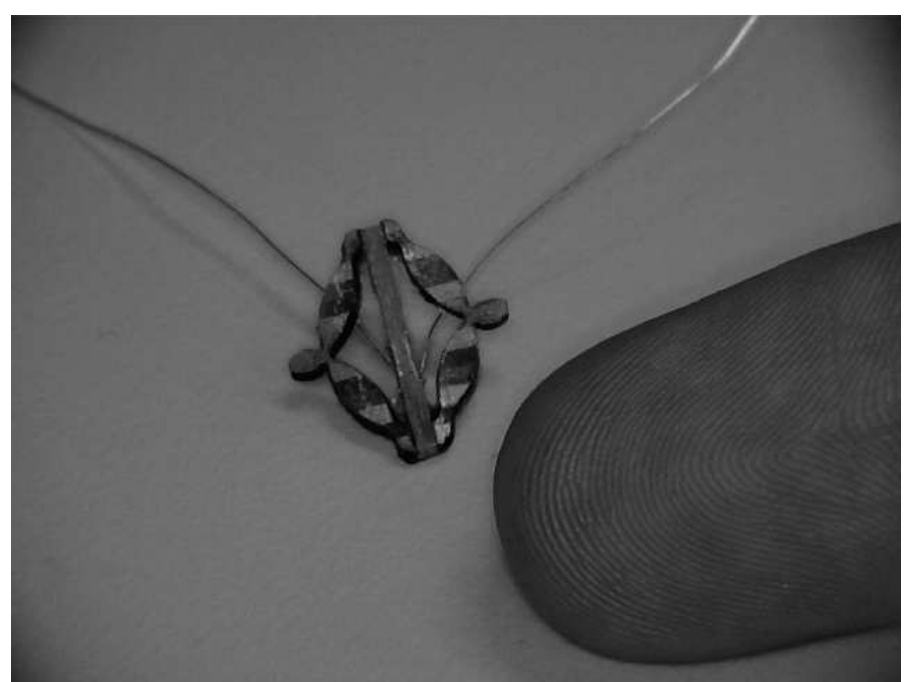

Figura 6.7: Condição de ensaio do protótipo de atuador gradado.

\subsubsection{Verificação Experimental}

Para aferir os deslocamentos gerados na face de atuação do protótipo, procede-se com o método descrito na Seção 4.5. Como o vibrômetro é destinado a medições de 
natureza dinâmica, o atuador é excitado a uma frequência de $4 k H z$, que aproxima o regime quasi-estático por ser distante das frequências de ressonância. Essa frequência é escolhida por ser inferior à primeira frequência de ressonância prevista para a estrutura, de acordo com a varredura em frequência (Figura 6.8) obtida pela simulação numérica feita através do software comercial COMSOL Multiphysics (COMSOL, INC., 2011). De fato, foi possível constatar experimentalmente a ausência de ressonâncias em torno desta frequência, através da variação da frequência de excitação.

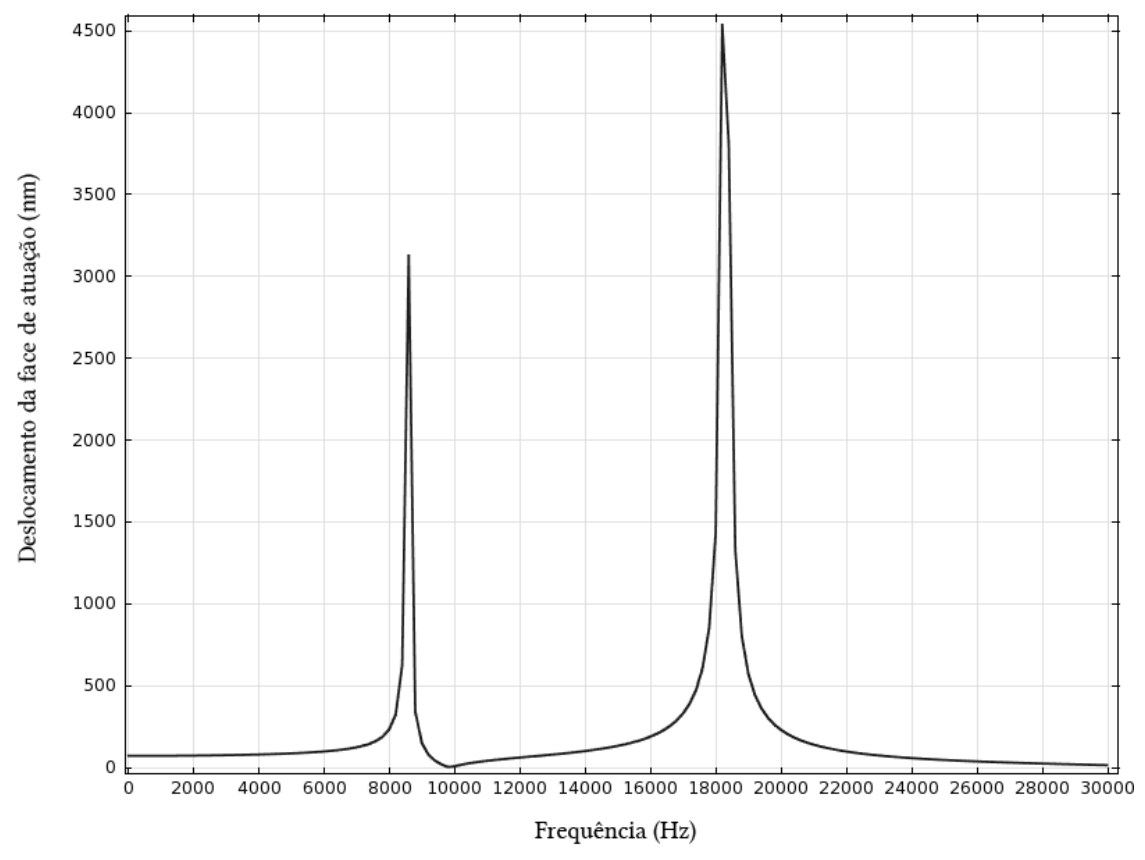

Figura 6.8: Deslocamento da face de atuação em função da frequência de excitação.

Assim, o protótipo foi ensaiado, como na montagem ilustrada na Figura 4.8, submetido a uma excitação com amplitude média de $18,2 \mathrm{~V}$ a $4 k H z$ produzida por um gerador de função Agilent 33250A (AGILENT TECHNOLOGIES, 2012) associado a um amplificador AR 800A3 (AR AMPLIFIERS, 2012). Utilizando o decodificador VD-09 do vibrômetro OFV-5000 (POLYTEC, 2011), a amplitude média de velocidade aferida pelo osciloscópio Agilent DSO3102A (AgILENT TEChNologies, 2012) foi $A_{v}=0,0078 \mathrm{~V}$. Como, pelas especificações do equipamento, $\varsigma_{v}=50 \mathrm{~mm} / \mathrm{s} / \mathrm{V}$, sabe-se que $A_{v}=0,39 \mathrm{~mm} / \mathrm{s}$. Então, através da relação dada na Equação (4.18), conclui-se que $A_{s}=15,52 \mathrm{~nm}$.

Pela simulação numérica, com a excitação da cerâmica em 18, $2 \mathrm{~V}$ a $4 k \mathrm{~Hz}$, a amplitude de deslocamento da face de atuação é de 80,39nm, como se observa pela deformada da Figura 6.9 . 


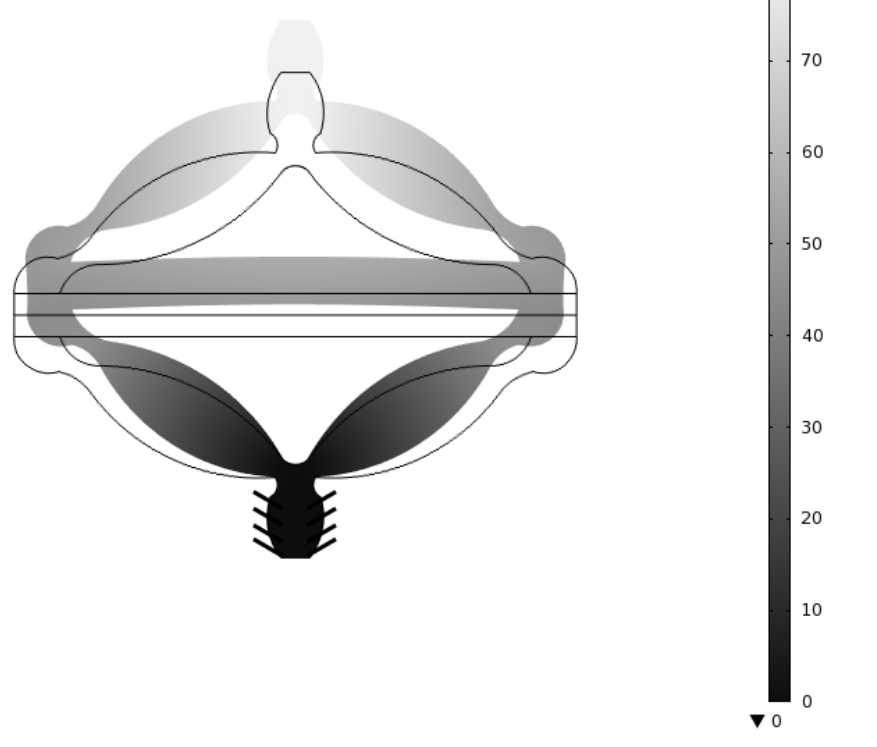

Figura 6.9: Deformada (em $\mathrm{mm}$ ) do protótipo sob excitação de $18,2 \mathrm{~V}$ a $4 k H z$.

\subsubsection{Discussão}

Numa gradação Cobre-Níquel, utilizar a aproximação dos módulos de rigidez enunciada pelas Equações (4.10) e (4.11) implica em tolerar um erro entre a rigidez considerada e a rigidez real da mistura. Considerando os módulos obtidos experimentalmente apresentados na Tabela 6.2, o máximo erro possível para cada proporção em volume é dado pela Figura 6.10. Assim, infere-se que para o módulo de compressibilidade $K$ o erro introduzido nunca é superior a 1,62\% e para o módulo de cisalhamento $G$ nunca é superior a 2,20\%. Sabe-se que a aproximação utilizada na Equação $(4.14$,$) para determinar a proporção em volume v_{N i}^{*}$ em cada camada da gradação, também podem introduzir discrepâncias. Porém, esses erros tem pouca significância, como é demonstrado pela Tabela 6.5.

Todavia, os erros acumulados pelas aproximações utilizadas nessa relação massa-rigidez tem pouca influência no distanciamento do modelo ao caso real, que se deve muito mais a erros inerentes à pesagem e mistura dos pós, ao processo de sinterização e à contaminação da amostra com material do molde.

No processo de verificação experimental do modelo numérico, nota-se uma grande discrepância nos deslocamentos observados na face de atuação do protótipo. Enquanto o modelo numérico indica 80,39nm, o que se observou no ensaio experimental foi um deslocamento de apenas 15,52nm, que representa um valor cerca de $80 \%$ menor. O modelo numérico já introduz variações pela desconsideração do amortecimento na estrutura e por considerar a porção metálica solidária à cerâmica ao invés de considerar a camada de cola, 


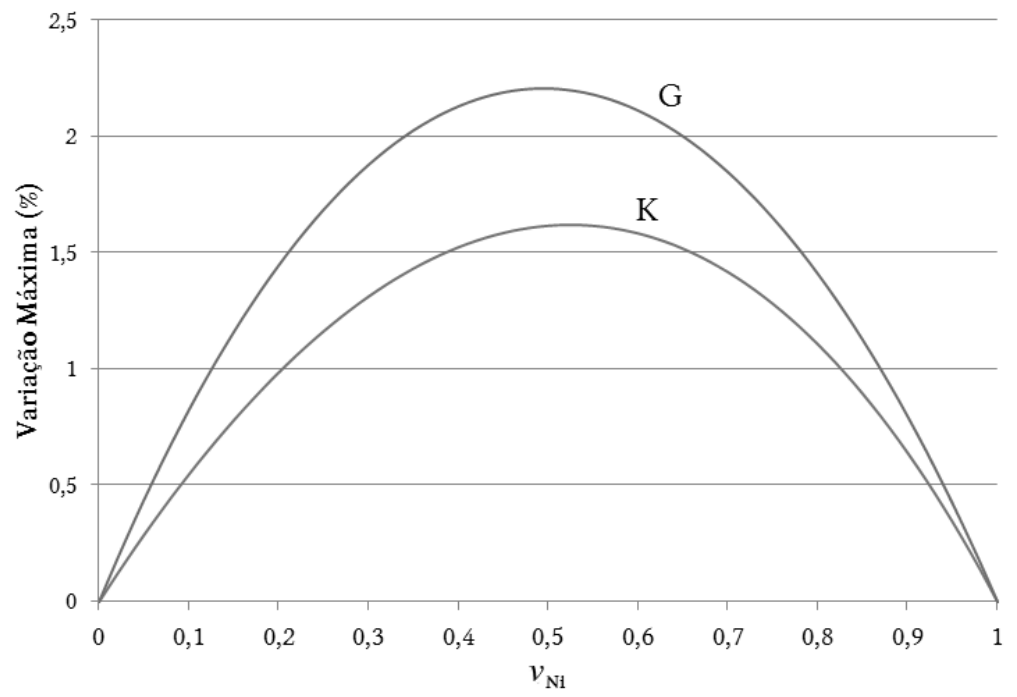

Figura 6.10: Variação máxima da rigidez conforme fração em volume de Níquel.

Tabela 6.5: Erro introduzido aos módulos de compressibilidade $(K)$ e de cisalhamento $(G)$ pela aproximação de material baseada no princípio variacional de Hashin-Shtrikman (HASHIN; SHTRIKMAN, 1963).

\begin{tabular}{cc|cc|cc|cc}
\hline Camada & $v_{N i}^{*}$ & $K_{\text {alvo }}(G P a)$ & $G_{a l v o}(G P a)$ & $K^{*}(G P a)$ & $G^{*}(G P a)$ & $\Delta K(\%)$ & $\Delta G(\%)$ \\
\hline 2 & 0,8952 & 149,10 & 68,68 & 148,72 & 68,86 & 0,2549 & 0,2621 \\
3 & 0,0238 & 75,29 & 31,29 & 75,25 & 31,31 & 0,0531 & 0,0639 \\
4 & 0,9670 & 158,51 & 73,44 & 158,36 & 73,51 & 0,0946 & 0,0953 \\
5 & 0,0550 & 77,10 & 32,20 & 77,00 & 32,25 & 0,1297 & 0,1553 \\
6 & 0,0032 & 74,11 & 30,69 & 74,11 & 30,69 & 0,0000 & 0,0000 \\
7 & 0,5894 & 116,28 & 52,05 & 115,59 & 52,40 & 0,5934 & 0,6724 \\
8 & 0,1991 & 86,04 & 36,73 & 85,71 & 36,90 & 0,3835 & 0,4628 \\
\hline
\end{tabular}

com suas devidas propriedades. Porém, ainda se acumulam a isto outros fatores intrínsecos ao processo de fabricação utilizado, como imperfeições na sinterização e no processo de colagem, sendo este último aprimorável através da utilização de adesivos mais adequados para esta finalidade, como o Loctite Tile Bond High Temp Red RTV (HENKELNA, 2012). 


\section{Conclusões e Trabalhos Futuros}

\subsection{Conclusões}

A formulação do MOT desenvolvida neste trabalho permite o projeto de mecanismos flexíveis constituídos por MGF com foco na manufatura. Esta formulação foi testada no projeto de atuadores e sensores piezelétricos, evidenciando que é possível melhorar o desempenho destes dispositivos com a agregação de gradações funcionais em suas estruturas acopladas. Pelos resultados obtidos, evidencia-se a vantagem de concentrar materiais mais flexíveis nas hinges de dispositivos flextensionais.

Porém, é possível notar que nem sempre materiais intermediários são a melhor alternativa, já que a resolução do problema de otimização, em alguns dos casos avaliados, levou a estruturas com regiões compostas puramente por um material ou por outro $\left(\rho_{2} \approx 0\right.$ ou $\left.\rho_{2} \approx 1\right)$. Este fato é evidente na garra com gradação em camadas perpendiculares à cerâmica (Figuras 5.11(b) e 5.12(a)) e no sensor com gradação em camadas paralelas à cerâmica (Figuras 5.19(b) e 5.20(a)). Todavia, a gradação de material continua sendo essencial para evitar concentrações de tensão nas interfaces entre materiais diferentes.

Para comprovar a viabilidade da fabricação destes dispositivos piezelétricos gradados, foi desenvolvido um processo de fabricação que combina a SJP com técnicas de microusinagem. Este processo foi validado através da fabricação de protótipos de atuadores piezelétricos, que apresentaram o funcionamento esperado, conforme demonstrado experimentalmente através de vibrômetro laser. Destaca-se como principal dificuldade do processo de fabricação, a etapa da colagem de cerâmicas piezelétricas a estruturas metálicas, tendo sido testadas diversas classes de adesivos sem a obtenção de resultados plenamente satisfatórios.

Assim, foi percorrido o ciclo completo de projeto, verificando as vantagens e a viabilidade da aplicação do conceito de MGF a dispositivos flextensionais piezelétricos nos âmbitos numérico e experimental. 


\subsection{Trabalhos Futuros}

Neste trabalho, o MOT foi implementado apenas para o projeto de estruturas estáticas, embora grande parte dos dispositivos piezelétricos tenham aplicação dinâmica. Assim, é natural a extensão da formulação desenvolvida para casos dinâmicos.

A formulação utilizada nos problemas de otimização deste trabalho se baseou na maximização de deslocamentos de saída ou na maximização do potencial elétrico de saída. Porém, ao utilizar esta abordagem observou-se uma tendência à formação de hinges (dobradiças) nas estruturas obtidas, ou seja, regiões extremamente delgadas e flexíveis que simulam juntas. Embora a técnica de projeção contorne este problema, pode ser interessante avaliar formulações alternativas que não busquem hinges. Cardoso e Fonseca (2004), por exemplo, apresentam uma formulação alternativa baseada na maximização da energia de deformação, que mitiga a ocorrência de hinges e pode ser uma abordagem interessante para os problemas de otimização deste trabalho.

No que se refere à fabricação, é importante melhorar a adesão de cerâmicas a estruturas metálicas ou fabricar gradações cerâmica-metal em peças inteiriças. Sabe-se que o processo desenvolvido é extensível a dispositivos piezelétricos integrais (CARBONARI; SILVA; PAULINO, 2010), visto que a SJP é capaz de produzir peças com gradação entre materiais cerâmicos e metálicos (RUBIO, 2010). Além disso, avanços recentes na técnica de EEF permitem a usinagem de materiais cerâmicos não-condutivos (KUCUKTURK; COGUN, 2010) e a técnica de CL produz bons resultados em materiais cerâmicos (BLACK; CHUA, 1997). Resta, então, que se trabalhe na obtenção de cerâmicas piezelétricas gradadas com materiais metálicos através da SJP.

Para fins de validação, é interessante a fabricação de lotes maiores de protótipos para dar base estatística à comparação entre os âmbitos numérico e experimental e para avaliar a reprodutibilidade na fabricação de dispositivos piezelétricos pelo processo de fabricação proposto. 


\section{Referências}

ADAMOWSKI, J. C.; BUIOCHI, F.; HIGUTI, R. T. Ultrasonic material characterization using large-aperture pvdf receivers. Ultrasonics, v. 50, p. 110-115, 2010.

AGILENT TECHNOLOGIES. 2012. Disponível em: <www.agilent.com>.

ALLIK, H.; HUGHES, T. J. R. Finite element method for piezoelectric vibration. International Journal for Numerical Methods in Engineering, v. 2, p. 151-157, 1970.

ALMAJID, A.; TAYA, M.; HUDNUT, S. Analysis of out-of-plane displacement and stress field in a piezocomposite plate with functionally graded microstructure. International Journal of Solids and Structures, v. 38, n. 19, p. 3377-3391, 2001.

AMIGO, R. C. R.; SILVA, E. C. N. Design of a flextensional piezoactuator based on functionally graded material concept using the topology optimization method. In: Proceedings of 21st International Congress of Mechanical Engineering. [S.l.: s.n.], 2011.

AMIGO, R. C. R.; VATANABE, S. L.; SILVA, E. C. N. Design, manufacturing and characterization of functionally graded flextensional piezoelectric actuators. Journal of Physics: Conference Series - Proceedings of 12th International Symposium on Multiscale, Multifunctional and Functionally Graded Materials, 2012.

ANDRADE, M. A. B. Análise de materiais piezelétricos compósitos para aplicações em transdutores de ultra-som. Dissertação (Mestrado) - Escola Politécnica da Universidade de São Paulo, 2006.

AR AMPLIFIERS. 2012. Disponível em: <www.arww-rfmicro.com>.

BENDSOE, M. P. Optimal shape design as a material distribution problem. Structural Optimization, v. 1, p. 192-202, 1989.

BENDSOE, M. P.; KIKUCHI, N. Generating optimal topologies in structural design using a homogenization method. Computer Methods in Applied Mechanics and Engineering, Elsevier Sequoia S. A., v. 71, p. 197-224, November 1988.

BLACK, I.; CHUA, K. L. Laser cutting of thick ceramic tile. Optics \& Laser Technology, v. 29, n. 4, p. 193-205, 1997.

BOURDIN, B. Filters in topology optimization. International Journal for Numerical Methods in Engineering, v. 50, p. 2143-2158, 2001.

BRUNS, T. E.; TORTORELLI, D. A. Topology optimization of non-linear elastic structures and compliant mechanisms. Computer Methods in Applied Mechanics and Engineering, v. 190, p. 3443-3459, 2001.

CALLISTER, W. D. Fundamentals of materials science and engineering. [S.1.]: John Wiley \& Sons, Inc, 2001. 
CARBOnARI, R. C.; NISHIWAKI, S.; PAUlinO, G. H.; SILVA, E. C. N. Piezoactuator design considering the optimum placement of fgm piezoelectric material. Proceedings of the Society of Photo-optical Instrumentations Engineers, v. 6523, p. 65230C, 2007.

CARBOnARI, R. C.; SILVA, E. C. N.; PAUlinO, G. H. Design of functionally graded piezoelectric actuators using topology optimization. Proceedings of the Society of Photo-optical Instrumentations Engineers, v. 6166, p. 616607, 2006.

CARBOnARI, R. C.; SILVA, E. C. N.; PAUlinO, G. H. Multi-actuated functionally graded piezoelectric micro-tools design: a multiphysics topology optimization approach. International Journal for Numerical Methods in Engineering, v. 77, p. 301-336, 2009.

CARBONARI, R. C.; SILVA, E. C. N.; PAULINO, G. H. Integral piezoactuator system with optimum placement of functionally graded material - a topology optimization paradigm. Journal of Intelligent Material Systems and Structures, v. 21, p. 1653-1668, 2010.

CARDOSO, E. L.; FONSECA, J. S. O. Strain energy maximization approach to the design of fully compliant mechanisms using the topology optimization. Latin American Journal of Solids and Structures, v. 1, p. 263-275, 2004.

CHU, C. L.; FAN, S. H. A novel long-travel piezoelectric-driven linear nanopositioning stage. Journal of the International Societies for Precision Engineering and Nanotechnology, v. 30, p. 85-95, 2006.

CHU, D. N.; XIE, Y. M.; HIRA, A.; STEVEN, G. P. Evolutionary structural optimization for problems with stiffness constraints. Finite Elements in Analysis and Design, v. 21, p. 239-251, 1996.

CLAEYSSEN, F.; LETTY, R. L.; BARILLOT, F.; LHERMET, N.; FABBRO, H.; GUAY, P.; YORCK, M.; BOUCHILLOUX, P. Mechanisms based on piezo actuators. Proceedings of Society of Photo-Optical Instrumentation Engineers, v. 4332, p. 225-233, 2001.

COMSOL, INC. COMSOL Multiphysics. 2011. Software. Disponível em: $<$ www.comsol.com $>$.

DAMJANOVIC, D. Ferroelectric, dielectric and piezoelectric properties of ferroelectric thin films and ceramics. Reports on Progress in Physics, v. 31, n. 3, p. 1267-1324, 1998.

DANTZIG, G. B. Linear programming and extensions. [S.l.]: Princeton, New Jersey, 1963.

DISCO CORPORATION. 2012. Disponível em:

$<$ http://www.disco.co.jp/eg/products/dicer/>.

EISINBERG, A.; MENCIASSI, A.; MICERA, S.; CAMPOLO, D.; CARROZZA, M.; DARIO, P. Pi force control of microgripper for assembling biomedical microdevices. IEEE Proceedings of Circuits Devices System, v. 148, p. 348-352, 2001.

ELKA, E.; ELATA, D.; ABRAMOVICH, H. The electromechanical response of mumultilayer piezoelectric structures. Journal of Microelectromechanical Systems, v. 13, n. 2 , p. $332-341,2004$.

HAERTLING, G. H. Rainbow ceramics: a new-type of ultra-high-displacement actuator. American Ceramic Society Bulletin, v. 73, p. 93-96, 1994.

HAFTKA, R. T.; GURDAL, Z.; KAMAT, M. P. Elements of structural optimization. 2. ed. [S.l.]: Kluwer Academic Publishers, 1990. 
HARTSUIJKER, C.; WELLEMAN, J. W. Engineering Mechanics. [S.l.]: Spinger, 2001.

HASHIN, Z.; SHTRIKMAN, S. A variational approach to the theory of the elastic behavior of multiphase materials. Journal of the Mechanics and Physics of Solids, v. 11, p. 127-140, 1963.

HE, J. H. Hamilton principle and generalized variational principles of linear thermopiezoelectricity. Journal of Applied Mechanics, v. 68, n. 4, p. 666-667, 2001 .

HENKELNA. Loctite Tile Bond High Temp Red RTV. 2012. Disponível em: $<$ www.henkelna.com $>$.

HUANG, M.; WANG, R.; THOMPSON, V.; REKOW, D.; SOBOYEJO, W. Bioinspired design of dental multilayers. Journal of Material Science: Materials in Medicine, v. 18, n. 1, p. 57-64, 2007.

HUGHES, T. J. R. The finite element method. [S.l.]: Englewood Cliffs, NY, 1987.

ICHINOSE, N.; MIYAMOTO, N.; TAKAHASHI, S. Ultrasonic transducers with functionally graded piezoelectric ceramics. Journal of the European Ceramic Society, v. 24, p. 1681-1685, 2004.

INDUSTRIAL LASER SOLUTIONS. Rotary stages for laser-machining tubes, stents. 2012. Disponível em: <http://www.industrial-lasers.com>.

IULA, A.; LAMBERTI, N.; PAPPALARDO, M. Analysis and experimental evaluation of a new planar piezoelectric accelerometer. IEEE Transactions on Mechatronics, v. 4, n. 2, p. 207-212, 1999.

JB QUíMICA. 2012. Disponível em: <www.jbquimica.com.br>.

JOG, C. S.; HABER, R. B. Stability of finite element models for distributed-parameter optimization and topology design. Computer Methods in Applied Mechanics and Engineering, v. 130, n. 3-4, p. 203-226, 1996.

KIEBACK, B.; NEUBRAND, A.; RIEDEL, H. Processing techniques of functionally graded materials. Materials Science and Engineering: A, v. 362, p. 81-105, 2003.

KIKUCHI, N.; HOLLISTER, S.; YOO, J. A concept of image-based integrational cae for production engineering. International Symposium on Optimization and Innovative Design, 1997.

KIYOnO, C. Y. Método de Otimização Topológica aplicado ao Projeto de Sonotrodos para Transdutores Piezelétricos. Dissertação (Mestrado) — Escola Politécnica da USP, 2008.

KJ LASER. Laser Cutting Gallery. 2012. Disponível em:

$<$ http://www.kjlasermicromachining.com>.

KOIZUMI, M. Fgm activities in japan. Composites Part B: Engineering, v. 28, p. 1-4, 1997.

KOSSOFF, G. The effects of backing and matching on the performance of piezoelectric ceramic transducers. IEEE Transactions of Sonic and Ultrasonic, v. 13, n. 1, p. 20-30, 1966. 
KUCUKTURK, G.; COGUN, C. A new method for machining of electrically nonconductive workpieces using electric discharge machining technique. Machining Science and Technology, v. 14, p. 189-207, 2010.

KURIHARA, K.; HIDA, M.; UMEMIYA, S.; KOGANEZAWA, S. Piezoelectric microactuators for use in magnetic disk drives. Japanese Journal of Applied Physics - Part 1, v. 43, p. $6725-6727,2004$.

KWON, K.; CHO, N.; JANG, W. The design and characterization of a piezo-driven inchworm linear motor with a reduction-lever mechanism. International Journal Series C-Mechanical Systems Machine Elements and Manufacturing, v. 47, p. 803-811, 2004.

LERCH, R. Simulation of piezoelectric devices by 2-dimensional and 3-dimensional finite elements. IEEE Transaction on Ultrasonics Ferroelectrics and Frequency Control, v. 37, p. 233-247, 1990.

LIU, Y.; TIAN, G.; WANG, Y.; LIN, J.; ZHANG, Q.; HOFMANN, H. F. Active piezoelectric energy harvesting: general principle and experimental demonstration. Journal of Intelligent Material Systems and Structures, v. 20, p. 575-585, 2009.

LOGITECH LTD. 2012. Disponível em: <www.logitech.uk.com>.

MATSUI, K.; TERADA, K. Continuos approximation of material distribution for topology optimization. International Journal for Numerical Methods in Engineering, v. 59, p. 1925-1944, 2004.

MITSUBISHI EDM LASER. eX Series. 2012. Disponível em: $<$ http://www.mitsubishi-world.com $>$.

MORGANITE BRASIL. 2012. Disponível em: <www.morganitebrasil.com.br>.

MOROZOV, A. K.; WEBB, D. C. A sound projector for acoustic tomography and global ocean monitoring. IEEE Journal of Oceanic Engineering, v. 27, p. 174-185, 2003.

NAILLON, M.; COURSANT, R. H.; BESNIER, F. Analysis of piezoelectric structures by a finite element method. Acta Eletronica, v. 25, n. 4, p. 341-362, 1983.

NAKASONE, P. H.; SILVA, E. C. N. Dynamic design of piezoelectric laminated sensors and actuators using topology optimization. Journal of Intelligent Material Systems and Structures, v. 21, p. 1627-1652, 2010.

NOVOTnY, A. A.; FEIJOO, R. A.; TAROCO, E.; PADRA, C. Topological sensitivity analysis. Computer Methods in Applied Mechanics and Engineering, v. 192, p. 803-829, 2003.

PAGE, J. H.; MCCULLOCH, R. D. Ultrasound propagation in sintered metal powder: evidence for a crossover from phonons to fractons. Physical Review Letters, v. 57, n. 11, p. 1324-1327, 1986.

POLYTEC. OFV-5000 Vibrometer Controller. 2011.

QIU, J.; TANI, J.; UENO, T.; MORITA, T.; TAKAHASHI, H.; DU, H. Fabrication and high durability of functionally graded piezoelectric bending actuators. Smart Materials and Structures, v. 12, n. 1, p. 115-121, 2003. 
REYNAERTS, D.; PEIRS, J.; BRUSSEL, H. van. A mechatronic approach to microsystem design. IEEE/ASME Transactions on Mechatronics, v. 3, p. 24-33, 1998.

RUBIO, W. M. Otimização topológica de transdutores piezelétricos com gradação funcional de material: projeto, simulação, análise e fabricação. Tese (Doutorado) — Escola Politécnica da Universidade de São Paulo, 2010.

RUBIO, W. M.; BUIOCHI, F.; ADAMOWSKI, J. C.; SILVA, E. C. N. Modeling of functionally graded piezoelectric ultrasonic transducers. Ultrasonics, v. 49, p. 484-494, 2009 .

RUPP, C. J.; EVGRAFOV, A.; MAUTE, K.; DUNN, M. L. Design of piezoelectric energy harvesting systems: a topology optimization approach based on multilayer plates and shells. Journal of Intelligent Material Systems and Structures, v. 20, p. 1923-1939, 2009.

SETHIAN, J. A.; WIEGMANN, A. Structural boundary design via level ser and immersed interface methods. Journal of Computational Physics, v. 163, p. 489-528, 2000.

SHIBATA, T.; UNNO, K.; MAKINO, E.; SHIMADA, S. Fabrication and characterization of diamond afm probe integrated with pzt thin film sensor and actuator. Sensors and Actuators A - Physical, v. 114, p. 398-405, 2004.

SHIN, K. H.; PARK, K. I.; KIM, Y.; SA-GONG, G. Vibrating sample magnometer using a multilayer piezoelectric actuator. Physica Status Solidi B: Basic Solid State Physics, v. 241, n. 7, p. 1633-1636, 2001.

SIGMUND, O. Design of multiphysics actuators using topology optimization - part ii: Two-material structures. Journal of Computer Methods in Applied Mechanics and Engineering, v. 190, p. 6605-6627, 2000.

SIGMUND, O.; PETERSSON, J. Numerical instabilities in topology optimization: a survey on procedures dealing with checkerboards, mesh-dependencies and local minima. Structural Optimization, v. 16, n. 1, p. 68-75, 1998.

SILVA, E. C. N.; NISHIWAKI, S.; KIKUCHI, N. Topology optimization design of flextensional actuators. IEEE Transaction on Ultrasonics Ferroelectrics and Frequency Control, v. 47, n. 3, p. 657-671, 2000.

SILVA, E. C. N.; WALTERS, M. C.; PAULINO, G. H. Modeling bamboo as a functionally graded material: lessons for the analysis of affordable materials. Journal of Materials Science, v. 41, p. 6991-7004, 2006.

SPS SYNTEX INC. Instruction Manual Model SPS-1050. [S.1.], 2008.

SVANBERG, K. The method of moving asymptotes - a new method for structural optimization. International Journal for Numerical Methods in Engineering, v. 24, p. 359-373, 1987.

SVANBERG, K. On the convexity and concavity of compliances. Structural and Multidisciplinary Optimization, v. 7, p. 42-46, 1994.

TAO, Y.; WU, X.; XIAO, D.; WU, Y.; CUI, H.; XI, X.; ZHU, B. Design, analysis and experiment of a novel ring vibratory gyroscope. Sensors and Actuators A: Physical, v. 168, p. 286-299, 2011. 
VANDERPLAATS, G. N. Numerical optimization techniques for engineering design: with applications. New York, EUA: McGraw-Hill, 1984.

WANG, H.; RITTER, T.; CAO, W.; SHUNG, K. K. High frequency properties of passive materials for ultrasonic transducers. IEEE Transactions on Ultrasonics, Ferroelectrics and Frequency Control, v. 48, n. 1, p. 78-84, 2001.

WIRE CUT COMPANY. EDM Machined Sample Parts-Wire. 2012. Disponível em: $<$ http://www.wirecutcompany.com>.

WOLFRAM RESEARCH. Wolfram Mathematica. 2012. Software. Disponível em: $<$ www.wolfram.com/mathematica $>$.

WORKSHOP OF PHOTONICS. Book Of Samples. 2012. Disponível em: $<$ http://www.wophotonics.com>.

WORONKO, A.; HUANG, J.; ALTINTAS, Y. Piezoelectric tool actuator for precision machining on conventional cnc turning center. Journal of the Societies for Precision Engineering and Nanotechnology, v. 27, p. 335-345, 2003.

ZHU, X.; MENG, Z. Operational principle, fabrication and displacement characteristics of functionally gradient piezoelectric ceramic actuator. Sensors and Actuators A, v. 48, p. 169-175, 1995. 


\section{Apêndice A - Piezeletricidade}

Piezeletricidade é a propriedade de certos materiais de gerar energia elétrica quando submetidos a esforços mecânicos. Essa denominação deriva do prefixo grego " $\pi \iota \varepsilon \sigma \eta "$ ", que significa "pressão". O efeito piezelétrico também se dá na forma reversa, ou seja, esses materiais apresentam a geração de forças mecânicas internas quando submetidos a um campo elétrico.

Alguns materiais naturais apresentam propriedades piezelétricas, exemplo de alguns cristais, como o quartzo, e de compostos orgânicos, como os ossos e o DNA. No entanto, alguns materiais artificiais com propriedades piezelétricas intensificadas foram desenvolvidos, como o Titanato Zircanato de Chumbo (PZT) e o Titanato de Bário $\left(\mathrm{BaTiO}_{3}\right)$. Nestes, o efeito piezelétrico é induzido artificialmente através de um processo de polarização, que torna as orientações dos grãos aproximadamente paralelas. Para isso, a cerâmica é inicialmente aquecida até a temperatura de Curie, acima da qual os dipolos presentes no material são maleáveis. A cerâmica é, então, submetida a um campo elétrico de grande magnitude (superior a 2000 V/mm), fazendo com que os dipolos se alinhem, como na Figura A.1(b). Por fim, o campo é mantido e o material é resfriado lentamente, tornando os dipolos da cerâmica aproximadamente alinhados, como na Figura A.1(c).

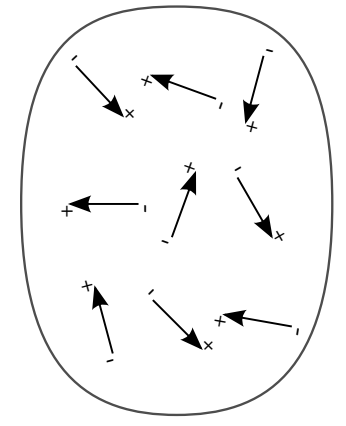

(a)

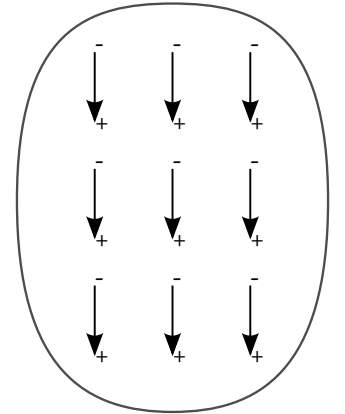

(b)

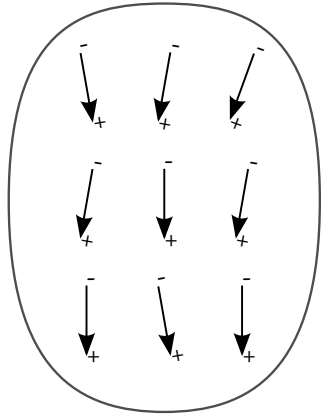

(c)

Figura A.1: Estado dos dipolos elétricos (a) antes, (b) durante e (c) após o processo de polarização.

A Figura A.2 representa como uma célula unitária de $\mathrm{BaTiO}_{3}$ se expande na direção paralela ao campo e se contrai na perpendicular. 


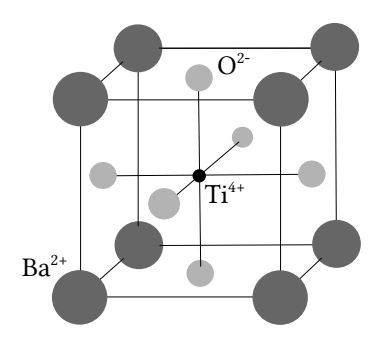

(a)
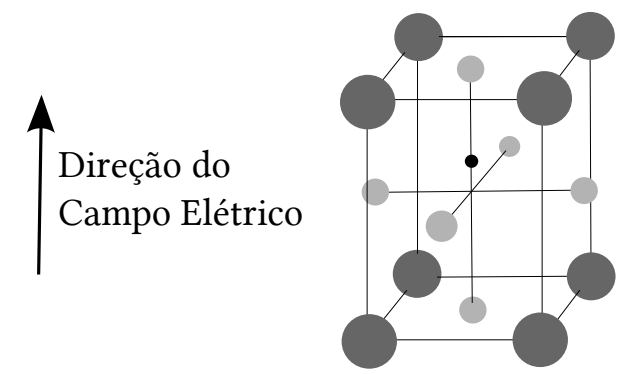

(b)

Figura A.2: Célula unitária de $\mathrm{BaTiO}_{3}$ (a) antes e (b) depois da polarização (CALLISTER, 2001).

As relações constitutivas piezelétricas relacionam grandezas mecânicas - geralmente a deformação $\mathbf{S}$ e a tensão $\mathbf{T}$ - com grandezas elétricas - geralmente o deslocamento elétrico $\mathbf{D}$ e o campo elétrico E. Embora essa relação não seja linear, é possivel utilizar uma aproximação pelos primeiros termos da série de Taylor correspondente, para expressar a dependência de $\mathbf{T}$ e $\mathbf{D}$ das variáveis $\mathbf{S}$ e $\mathbf{E}$ na seguinte forma:

$$
\left\{\begin{array}{l}
\mathbf{T}=\left(\frac{\partial \mathbf{T}}{\partial \mathbf{S}}\right)_{\mathbf{E}} \mathbf{S}+\left(\frac{\partial \mathbf{T}}{\partial \mathbf{E}}\right)_{\mathbf{S}} \mathbf{E} \\
\mathbf{D}=\left(\frac{\partial \mathbf{D}}{\partial \mathbf{S}}\right)_{\mathbf{E}} \mathbf{S}+\left(\frac{\partial \mathbf{D}}{\partial \mathbf{E}}\right)_{\mathbf{S}} \mathbf{E}
\end{array}\right.
$$

onde as derivadas parciais com os subscritos $\mathbf{E}$ e $\mathbf{S}$ são calculadas, respectivamente, a campo elétrico constante e a deformação mecânica constante.

Lerch (1990) reescreve as relações anteriores de forma mais usual:

$$
\left\{\begin{array}{l}
\mathbf{T}=\mathbf{c}_{E} \mathbf{S}-\mathbf{e}^{t} \mathbf{E} \\
\mathbf{D}=\mathbf{e S}+\varepsilon_{S} \mathbf{E}
\end{array}\right.
$$

onde $\mathbf{c}_{E}$, e e $\varepsilon_{S}$ são, respectivamente, as matrizes elástica, piezelétrica e dielétrica, dadas a seguir.

$$
\begin{aligned}
\mathbf{c}_{E} & =\left[\begin{array}{cccccc}
c_{11} & c_{12} & c_{13} & 0 & 0 & 0 \\
c_{12} & c_{11} & c_{13} & 0 & 0 & 0 \\
c_{13} & c_{13} & c_{33} & 0 & 0 & 0 \\
0 & 0 & 0 & c_{44} & 0 & 0 \\
0 & 0 & 0 & 0 & c_{44} & 0 \\
0 & 0 & 0 & 0 & 0 & c_{66}
\end{array}\right] \\
\mathbf{e} & =\left[\begin{array}{cccccc}
0 & 0 & 0 & 0 & e_{15} & 0 \\
0 & 0 & 0 & e_{15} & 0 & 0 \\
e_{31} & e_{31} & e_{33} & 0 & 0 & 0
\end{array}\right]
\end{aligned}
$$




$$
\varepsilon_{S}=\left[\begin{array}{ccc}
\varepsilon_{11} & 0 & 0 \\
0 & \varepsilon_{11} & 0 \\
0 & 0 & \varepsilon_{33}
\end{array}\right]
$$

$\mathrm{Na}$ formulação apresentada, os materiais piezelétricos são elástica e piezeletricamente anisotrópicos na direção de polarização 3 (independentemente de coincidir com a direção $y$ ou $z$ ) e isotrópicos no plano 12. Como neste trabalho os problemas analisados são bidimensionais, a Equação A.6 faz a direção de polarização coincidir com y:

$$
\left\{\begin{array}{c}
T_{x x} \\
T_{y y} \\
T_{z z} \\
T_{y z} \\
T_{x z} \\
T_{x y} \\
\hline D_{x} \\
D_{y} \\
D_{z}
\end{array}\right\}=\left[\begin{array}{cccccc|ccc}
c_{11} & c_{13} & c_{12} & 0 & 0 & 0 & 0 & 0 & e_{31} \\
c_{13} & c_{33} & c_{13} & 0 & 0 & 0 & 0 & 0 & e_{33} \\
c_{12} & c_{13} & c_{11} & 0 & 0 & 0 & 0 & 0 & e_{31} \\
0 & 0 & 0 & c_{44} & 0 & 0 & 0 & e_{15} & 0 \\
0 & 0 & 0 & 0 & c_{66} & 0 & 0 & 0 & 0 \\
0 & 0 & 0 & 0 & 0 & c_{44} & e_{15} & 0 & 0 \\
\hline 0 & 0 & 0 & 0 & 0 & e_{15} & -\varepsilon_{11} & 0 & 0 \\
0 & 0 & 0 & e_{15} & 0 & 0 & 0 & -\varepsilon_{11} & 0 \\
e_{31} & e_{33} & e_{31} & 0 & 0 & 0 & 0 & 0 & -\varepsilon_{33}
\end{array}\right]\left\{\begin{array}{c}
S_{x x} \\
S_{y y} \\
S_{z z} \\
S_{y z} \\
S_{x z} \\
S_{x y} \\
-E_{x} \\
-E_{y} \\
-E_{z}
\end{array}\right\}
$$




\section{Apêndice B - Método dos Elementos Finitos Piezelétrico}

O Método dos Elementos Finitos (MEF) é uma técnica computacional empregada na resolução de problemas regidos por equações diferenciais e sujeitos a determinadas condições de contorno. Nesse método, o domínio é dividido em subdomínios, ditos elementos finitos, dentro dos quais as equações diferenciais governantes são aproximadas por funções mais simples, chamadas funções de forma.

\section{B.1 Princípio Variacional Piezelétrico}

Este trabalho utiliza o princípio variacional piezelétrico para determinar a relação entre as variáveis nodais e as condições de contorno do problema. As equações dinâmicas do meio piezelétrico contínuo provêm do princípio de Hamilton (NAILLON; COURSANT; BESNIER, 1983) e são dadas por:

$$
\delta \int_{t_{1}}^{t_{2}}(\mathcal{L}+\mathcal{W}) d t=0
$$

onde $t_{1}$ e $t_{2}$ definem o intervalo de tempo, $\mathcal{L}$ denota o lagrangiano e $\mathcal{W}$ denota o trabalho virtual das carregamentos externos mecânicos e elétricos. Sendo que, pelo princípio dos trabalhos virtuais, $t_{1}=t$ e $t_{2}=t$.

No caso piezelétrico, o lagrangiano $\mathcal{L}$ é dado por (HE, 2001):

$$
\mathcal{L}=\mathcal{T}+\mathcal{H}
$$

onde $\mathcal{T}$ denota a energia cinética associada e $\mathcal{H}$ denota a entalpia elétrica, que corresponde ao funcional de Gibbs (DAMJANOVIC, 1998) e equivale à energia potencial $\mathcal{V}$ do sistema.

Sendo assim, tomando por base a Equação (A.2), tem-se que (HE, 2001):

$$
\mathcal{H}=\frac{\mathbf{S}^{t} \mathbf{T}-\mathbf{E}^{t} \mathbf{D}}{2}
$$


A energia cinética $\mathcal{T}$ é definida por Naillon, Coursant e Besnier (1983) como:

$$
\mathcal{T}=\frac{\varrho \dot{\mathbf{u}}^{t} \dot{\mathbf{u}}}{2}
$$

onde $\varrho$ denota a densidade do meio e $\dot{\mathbf{u}}$ o campo de velocidades.

Na entalpia elétrica $\mathcal{H}$, pela aplicação do operador variacional, tem-se:

$$
\delta \mathcal{H}=\delta \mathbf{S}^{t}\left(\mathbf{c}^{E} \mathbf{S}-\mathbf{e}^{t} \mathbf{E}\right)-\delta \mathbf{E}^{t}\left(\mathbf{e S}+\boldsymbol{\varepsilon}^{S} \mathbf{E}\right)
$$

Já na energia cinética $\mathcal{T}$, a primeira variação é dada por:

$$
\delta \mathcal{T}=\varrho \delta \dot{\mathbf{u}}^{t} \dot{\mathbf{u}}
$$

que, ao realizar uma integração por partes, leva a:

$$
\int_{t_{1}}^{t_{2}} \delta \mathcal{T} d t=\int_{t_{1}}^{t_{2}}\left(\varrho \delta \dot{\mathbf{u}}^{t} \dot{\mathbf{u}}\right) d t=\left.\varrho \delta \mathbf{u}^{t} \dot{\mathbf{u}}\right|_{t_{1}} ^{t_{2}}-\int_{t_{1}}^{t_{2}}\left(\varrho \delta \mathbf{u}^{t} \ddot{\mathbf{u}}\right) d t
$$

onde o primeiro termo vai a zero, visto que $\delta \mathbf{u}=0$ em $t=t_{1}$ e $t=t_{2}$.

Logo, a variação da energia cinética vale:

$$
\delta \mathcal{T}=-\varrho \delta \mathbf{u}^{t} \ddot{\mathbf{u}}
$$

Portanto, o lagrangiano $\mathcal{L}$ pode ser expresso como:

$$
\delta \mathcal{L}=\int_{t_{1}}^{t_{2}} \int_{V}\left(-\varrho \delta \mathbf{u}^{t} \ddot{\mathbf{u}}+\delta \mathbf{S}^{t}\left(\mathbf{c}^{E} \mathbf{S}-\mathbf{e}^{t} \mathbf{E}\right)-\delta \mathbf{E}^{t}\left(\mathbf{e S}+\varepsilon^{S} \mathbf{E}\right)\right) d V d t
$$

Por sua vez, o trabalho virtual $\delta \mathcal{W}$ realizado pelos carregamentos pontuais mecânicos $\mathbf{f}$ e elétricos q na variação do campo de deslocamento $\delta \mathbf{u}$ e de potencial elétrico $\delta \phi$ é dado por:

$$
\delta \mathcal{W}=\int_{t_{1}}^{t_{2}}\left(\delta \mathbf{u}^{t} \mathbf{f}-\delta \boldsymbol{\phi}^{t} \mathbf{q}\right) d t
$$

Com isso, o variacional resultante é dado por (ALLIK; HUGHES, 1970):

$$
\int_{V}\left(-\varrho \delta \mathbf{u}^{t} \ddot{\mathbf{u}}+\delta \mathbf{S}^{t}\left(\mathbf{c}^{E} \mathbf{S}-\mathbf{e}^{t} \mathbf{E}\right)-\delta \mathbf{E}^{t}\left(\mathbf{e} \mathbf{S}+\varepsilon^{S} \mathbf{E}\right)\right) d V+\delta \mathbf{u}^{t} \mathbf{f}-\delta \boldsymbol{\phi}^{t} \mathbf{q}=0
$$

De modo que, finalmente, obtêm-se as equações de equilíbrio piezelétricas, expressas por:

$$
\begin{cases}\int_{V}\left(\varrho \delta \mathbf{u}^{t} \ddot{\mathbf{u}}+\delta \mathbf{S}^{t}\left(\mathbf{c}^{E} \mathbf{S}-\mathbf{e}^{t} \mathbf{E}\right)\right) d V & =\delta \mathbf{u}^{t} \mathbf{f} \\ \int_{V}\left(\delta \mathbf{E}^{t}\left(\mathbf{e} \mathbf{S}+\varepsilon^{S} \mathbf{E}\right)\right) d V & =\delta \boldsymbol{\phi}^{t} \mathbf{q}\end{cases}
$$

Porém, nos problemas de otimização estudados neste trabalho, são incluídas molas nas faces de atuação/sensoriamento $\Gamma_{t}$, visando a garantia de uma rigidez estrutural mínima. 
Sendo $k_{m}$ a rigidez dessas molas, a energia potencial $\mathcal{V}$ torna-se:

$$
\mathcal{V}=\mathcal{H}+\frac{\mathbf{u}_{\Gamma_{t}}^{t} k_{m} \mathbf{u}_{\Gamma_{t}}}{2}
$$

Além disso, este trabalho considera apenas o caso estático. Portanto, as equações de equilíbrio piezelétrico tornam-se:

$$
\begin{cases}\int_{V}\left(\delta \mathbf{S}^{t}\left(\mathbf{c}^{E} \mathbf{S}-\mathbf{e}^{t} \mathbf{E}\right) \delta \mathbf{u}+k_{m} \mathbf{u}_{\Gamma_{t}} \delta \mathbf{u}_{\Gamma_{t}}\right) d V & =\delta \mathbf{u}^{t} \mathbf{f} \\ \int_{V}\left(\delta \mathbf{E}^{t}\left(\mathbf{e} \mathbf{S}+\boldsymbol{\varepsilon}^{S} \mathbf{E}\right)\right) d V & =\delta \boldsymbol{\phi}^{t} \mathbf{q}\end{cases}
$$

\section{B.2 Estado Plano de Tensões Mecânicas}

A formulação de MEF deste trabalho considera a situação de Estado Plano de Tensões Mecânicas (EPTM). Essa aproximação se aplica aos casos onde duas dimensões do domínio possuem dimensões de mesma grandeza, mas muito superiores à da terceira, conforme mostrado na Figura B.1

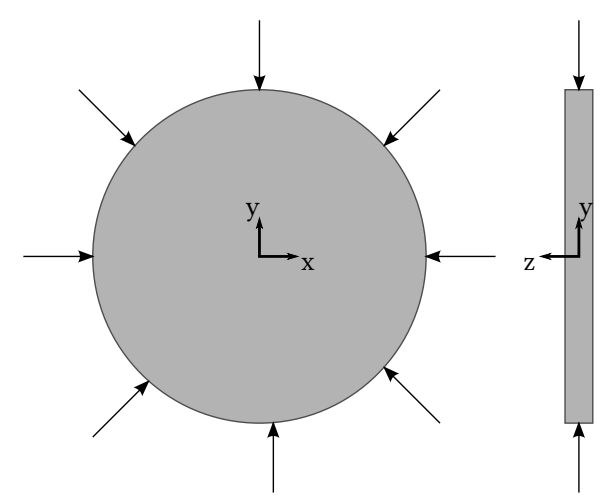

Figura B.1: Estado Plano de Tensões Mecânicas.

Nessa situação, é razoável considerar a seguinte hipótese:

$$
T_{z z}=T_{x z}=T_{y z}=D_{z}=0
$$

Logo, para o material não-piezelétrico isotrópico, as relações elásticas se tornam:

$$
\left\{\begin{array}{l}
T_{x x} \\
T_{y y} \\
T_{x y}
\end{array}\right\}=\frac{E}{(1+\nu)(1-2 \nu)}\left[\begin{array}{ccc}
1-\nu & \nu & 0 \\
\nu & 1-\nu & 0 \\
0 & 0 & \frac{1-2 \nu}{2}
\end{array}\right]\left\{\begin{array}{c}
S_{x x} \\
S_{y y} \\
S_{x y}
\end{array}\right\}
$$


Para o material piezelétrico anisotrópico, a Equação A.6 se torna:

$$
\left\{\begin{array}{l}
T_{x x} \\
T_{y y} \\
T_{x y} \\
D_{x} \\
D_{y}
\end{array}\right\}=\left[\begin{array}{ccccc}
c_{11}-\frac{c_{12}^{2}}{c_{11}} & c_{13}-\frac{c_{12} c_{13}}{c_{11}} & 0 & 0 & e_{31}-\frac{e_{31} c_{12}}{c_{11}} \\
c_{13}-\frac{c_{12} c_{13}}{c_{11}} & c_{33}-\frac{c_{13}^{2}}{c_{11}} & 0 & 0 & e_{33}-\frac{e_{31} c_{13}}{c_{11}} \\
0 & 0 & c_{66} & e_{15} & 0 \\
0 & 0 & e_{15} & -\varepsilon_{11} & 0 \\
e_{31}-\frac{e_{31} c_{12}}{c_{11}} & e_{33}-\frac{e_{31} c_{13}}{c_{11}} & 0 & 0 & -\varepsilon_{33}-\frac{e_{31}^{2}}{c_{11}}
\end{array}\right]\left\{\begin{array}{c}
S_{x x} \\
S_{y y} \\
S_{x y} \\
-E_{x} \\
-E_{y}
\end{array}\right\}
$$

\section{B.3 Elemento Isoparamétrico de Quatro Nós}

No software desenvolvido neste trabalho, foi implementado o elemento isoparamétrico de quatro nós. Nele, os deslocamentos mecânicos $\mathbf{u}_{e}=\left\{u_{e_{x}} u_{e_{y}}\right\}^{t}$ e o potencial elétrico $\phi_{e}$ do elemento são calculados a partir de seus valores nodais, dados pelos vetores $\{\mathbf{U}\}_{e}$ e $\{\boldsymbol{\Phi}\}_{e}$.

$$
\begin{aligned}
\mathbf{u}_{e} & =\left[\mathbf{N}_{\mathbf{u}}\right]^{t}\{\mathbf{U}\}_{e} \\
\phi_{e} & =\left[\mathbf{N}_{\phi}\right]^{t}\{\boldsymbol{\Phi}\}_{e}
\end{aligned}
$$

onde os vetores das funções de forma, $\left[\mathbf{N}_{\mathbf{u}}\right]$ e $\left[\mathbf{N}_{\phi}\right]$, são dados por:

$$
\begin{gathered}
{\left[\mathbf{N}_{\mathbf{u}}\right]=\left[\begin{array}{cccccccc}
N_{1} & 0 & N_{2} & 0 & N_{3} & 0 & N_{4} & 0 \\
0 & N_{1} & 0 & N_{2} & 0 & N_{3} & 0 & N_{4}
\end{array}\right]} \\
{\left[\mathbf{N}_{\phi}\right]=\left[\begin{array}{lllll}
N_{1} & N_{2} & N_{3} & N_{4}
\end{array}\right]}
\end{gathered}
$$

onde as funções de forma $N_{i}$ são funções de interpolação de Lagrange, definidas em termos de coordenadas locais normalizadas, sendo que $-1 \leq \xi, \eta \leq 1$.

$$
\begin{aligned}
& N_{1}(\xi, \eta)=\frac{(1-\xi)(1-\eta)}{4} \\
& N_{2}(\xi, \eta)=\frac{(1+\xi)(1-\eta)}{4} \\
& N_{3}(\xi, \eta)=\frac{(1+\xi)(1+\eta)}{4} \\
& N_{4}(\xi, \eta)=\frac{(1-\xi)(1+\eta)}{4}
\end{aligned}
$$

A transformação entre os sistemas de coordenadas é feita pela matriz Jacobiana $[\mathbf{J}]$ da seguinte forma:

$$
\left\{\begin{array}{l}
d x \\
d y
\end{array}\right\}=[\mathbf{J}]^{t}\left\{\begin{array}{c}
d \xi \\
d \eta
\end{array}\right\}
$$


onde:

$$
[\mathbf{J}]=\left[\begin{array}{ll}
\frac{\delta x}{\delta \xi} & \frac{\delta y}{\delta \xi} \\
\frac{\delta x}{\delta \eta} & \frac{\delta y}{\delta \eta}
\end{array}\right]
$$

Calculados os deslocamentos mecânicos e os potenciais elétricos, é possivel obter o vetor deformação mecânica $\mathbf{S}$ e o vetor campo elétrico $\mathbf{E}$ tendo em vista que:

$$
\begin{aligned}
& \mathbf{S}=\left[\begin{array}{lll}
\frac{\partial u_{x}}{\partial x} & \frac{\partial u_{y}}{\partial y} & \left(\frac{\partial u_{x}}{\partial y}+\frac{\partial u_{y}}{\partial x}\right)
\end{array}\right]^{t} \\
& \mathbf{E}=-\left[\begin{array}{ll}
\frac{\partial \phi}{\partial x} & \frac{\partial \phi}{\partial y}
\end{array}\right]^{t}
\end{aligned}
$$

Logo, pelas Equações (B.18) e (B.19), tem-se que:

$$
\begin{gathered}
\mathbf{S}=\left[\mathbf{B}_{\mathbf{u}}\right]\{\mathbf{U}\}_{e} \\
\mathbf{E}=-\left[\mathbf{B}_{\phi}\right]\{\boldsymbol{\Phi}\}_{e}
\end{gathered}
$$

onde:

$$
\begin{gathered}
{\left[\mathbf{B}_{\mathbf{u}}\right]=\left[\begin{array}{cc}
\frac{\delta}{\delta x} & 0 \\
0 & \frac{\delta}{\delta y} \\
\frac{\delta}{\delta y} & \frac{\delta}{\delta x}
\end{array}\right]\left[\mathbf{N}_{\mathbf{u}}\right]} \\
{\left[\mathbf{B}_{\phi}\right]=\left[\begin{array}{c}
\frac{\delta}{\delta x} \\
\frac{\delta}{\delta y}
\end{array}\right]\left[\mathbf{N}_{\phi}\right]}
\end{gathered}
$$

\section{B.4 Formulação Matricial do Elemento}

Uma vez definidas as propriedades do elemento isoparamétrico utilizado, é possível definir sua formulação matricial. Para isso, utiliza-se o conceito dos trabalhos virtuais, pelo qual:

$$
\begin{array}{r}
\delta \mathbf{S}=\left[\mathbf{B}_{\mathbf{u}}\right]\{\delta \mathbf{U}\}_{e} \\
\delta \mathbf{E}=-\left[\mathbf{B}_{\phi}\right]\{\delta \boldsymbol{\Phi}\}_{e}
\end{array}
$$

Assim, as relações da Equação (B.12) podem ser expressas como:

$$
\left\{\begin{array}{c}
\{\delta \mathbf{U}\}_{e}^{t} \int_{V_{e}}\left(\begin{array}{c}
\varrho\left[\mathbf{N}_{\mathbf{u}}\right]^{t}\left[\mathbf{N}_{\mathbf{u}}\right]\{\ddot{\mathbf{U}}\}_{e}+ \\
+\left[\mathbf{B}_{\mathbf{u}}\right]^{t}\left[\mathbf{c}^{E}\right]\left[\mathbf{B}_{\mathbf{u}}\right]\{\mathbf{U}\}_{e}+ \\
+\left[\mathbf{B}_{\mathbf{u}}\right]^{t}[\mathbf{e}]^{t}\left[\mathbf{B}_{\phi}\right]\{\boldsymbol{\Phi}\}_{e}
\end{array}\right) d V_{e}=\{\delta \mathbf{U}\}_{e}^{t}\left[\mathbf{N}_{\mathbf{u}}\right]^{t}\{\mathbf{f}\}_{e} \\
\{\delta \boldsymbol{\Phi}\}_{e}^{t} \int_{V_{e}}\left(\begin{array}{c}
{\left[\mathbf{B}_{\phi}\right]^{t}[\mathbf{e}]\left[\mathbf{B}_{\mathbf{u}}\right]\{\mathbf{U}\}_{e}-} \\
-\left[\mathbf{B}_{\phi}\right]^{t}\left[\boldsymbol{\varepsilon}^{S}\right]\left[\mathbf{B}_{\phi}\right]\{\boldsymbol{\Phi}\}_{e}
\end{array}\right) d V_{e}=\{\delta \boldsymbol{\Phi}\}_{e}^{t}\left[\mathbf{N}_{\phi}\right]^{t}\{\mathbf{q}\}_{e}
\end{array}\right.
$$


de onde se define que:

$$
\begin{aligned}
& {\left[\mathbf{K}_{\mathbf{u u}}\right]_{e}=\int_{V_{e}}\left[\mathbf{B}_{\mathbf{u}}\right]^{t}\left[\mathbf{c}^{E}\right]\left[\mathbf{B}_{\mathbf{u}}\right] d V_{e}} \\
& {\left[\mathbf{K}_{\mathbf{u} \phi}\right]_{e}=\int_{V_{e}}\left[\mathbf{B}_{\mathbf{u}}\right]^{t}[\mathbf{e}]^{t}\left[\mathbf{B}_{\phi}\right] d V_{e}} \\
& {\left[\mathbf{K}_{\phi \phi}\right]_{e}=\int_{V_{e}}\left[\mathbf{B}_{\phi}\right]^{t}\left[\boldsymbol{\varepsilon}^{S}\right]\left[\mathbf{B}_{\phi}\right] d V_{e}}
\end{aligned}
$$

Hughes (1987) define a operação de montagem de uma matriz $\mathbf{M}$ de elementos finitos qualquer, a partir de matrizes elementares $\mathbf{m}_{e}$ como:

$$
\mathbf{M}=\bigwedge_{e=1}^{N} \mathbf{m}_{e}
$$

onde $N$ é o número total de elementos e $\bigwedge$ é denominado operador montagem.

Portanto, a equação linear estática do MEF piezelétrico é dada por:

$$
\left[\begin{array}{cc}
\mathbf{K}_{\mathbf{u u}} & \mathbf{K}_{\mathbf{u} \phi} \\
\mathbf{K}_{\phi \mathbf{u}} & -\mathbf{K}_{\phi \phi}
\end{array}\right]\left\{\begin{array}{c}
\mathbf{U} \\
\mathbf{\Phi}
\end{array}\right\}=\left\{\begin{array}{c}
\mathbf{F} \\
\mathbf{Q}
\end{array}\right\}
$$

onde:

$$
\begin{aligned}
{\left[\mathbf{K}_{\mathbf{u u}}\right] } & =\bigwedge_{e=1}^{N}\left[\mathbf{K}_{\mathbf{u u}}\right]_{e} \\
{\left[\mathbf{K}_{\mathbf{u} \phi}\right] } & =\bigwedge_{e=1}^{N}\left[\mathbf{K}_{\mathbf{u} \phi}\right]_{e} \\
{\left[\mathbf{K}_{\phi \phi}\right] } & =\bigwedge_{e=1}^{N}\left[\mathbf{K}_{\phi \phi}\right]_{e}
\end{aligned}
$$

\section{B.5 Prescrição de Deslocamentos}

Conhecendo a matriz de rigidez total e o vetor de carregamentos, determina-se o vetor de deslocamentos nodais. Porém, caso seja necessário prescrever o valor do potencial elétrico de determinados nós, é preciso enunciar um novo problema linear.

Inicialmente, o sistema da Equação (B.36) é reescrito como:

$$
\left[\begin{array}{ccc}
\mathbf{K}_{\mathbf{u u}} & \mathbf{K}_{\mathbf{u} \phi_{l}} & \mathbf{K}_{\mathbf{u} \phi_{p}} \\
\mathbf{K}_{\phi_{l} \mathbf{u}} & \mathbf{K}_{\phi_{l} \phi_{l}} & \mathbf{K}_{\phi_{l} \phi_{p}} \\
\mathbf{K}_{\phi_{p} \mathbf{u}} & \mathbf{K}_{\phi_{p} \phi_{l}} & \mathbf{K}_{\phi_{r} \phi_{p}}
\end{array}\right]\left\{\begin{array}{c}
\mathbf{U} \\
\boldsymbol{\Phi}_{l} \\
\boldsymbol{\Phi}_{p}
\end{array}\right\}=\left\{\begin{array}{c}
\mathbf{F} \\
\mathbf{Q}_{l} \\
\mathbf{Q}_{p}
\end{array}\right\}
$$

onde $\boldsymbol{\Phi}_{p}$ são os potenciais elétricos prescritos e $\boldsymbol{\Phi}_{l}$ os livres.

Considerando as condições de contorno de Dirichlet e sabendo-se que $\mathbf{Q}_{l}=\mathbf{0}$, por se tratarem de cargas internas, o novo sistema é dado por:

$$
\left[\begin{array}{ccc}
\mathbf{K}_{\mathbf{u u}} & \mathbf{K}_{\mathbf{u} \phi_{l}} & \mathbf{0} \\
\mathbf{K}_{\phi_{l} \mathbf{u}} & \mathbf{K}_{\phi_{l} \phi_{l}} & \mathbf{0} \\
\mathbf{0} & \mathbf{0} & \mathbf{I}
\end{array}\right]\left\{\begin{array}{c}
\mathbf{U} \\
\boldsymbol{\Phi}_{l} \\
\boldsymbol{\Phi}_{p}
\end{array}\right\}=\left\{\begin{array}{c}
\mathbf{F} \\
\mathbf{0} \\
\mathbf{0}
\end{array}\right\}+\left\{\begin{array}{c}
-\mathbf{K}_{\mathbf{u} \phi_{p}} \boldsymbol{\Phi}_{p} \\
-\mathbf{K}_{\phi_{l} \phi_{p}} \boldsymbol{\Phi}_{p} \\
\boldsymbol{\Phi}_{p}
\end{array}\right\}
$$




\section{B.6 Verificação da Implementação do MEF}

Tendo em vista a verificação do MEF implementado pelo software desenvolvido via MATLAB (denominado AMIGOT), nesta Seção o mesmo problema é resolvido neste e no software COMSOL (COMSOL, INC., 2011). O domínio controle é idêntico ao da Figura 5.1, porém com dimensão de altura igual a $5 \mathrm{~mm}$ ao invés de $6 \mathrm{~mm}$. Aplica-se um potencial elétrico de $-10 \mathrm{~V}$ ao eletrodo livre. A deformada obtida via COMSOL é apresentada na Figura B.2.

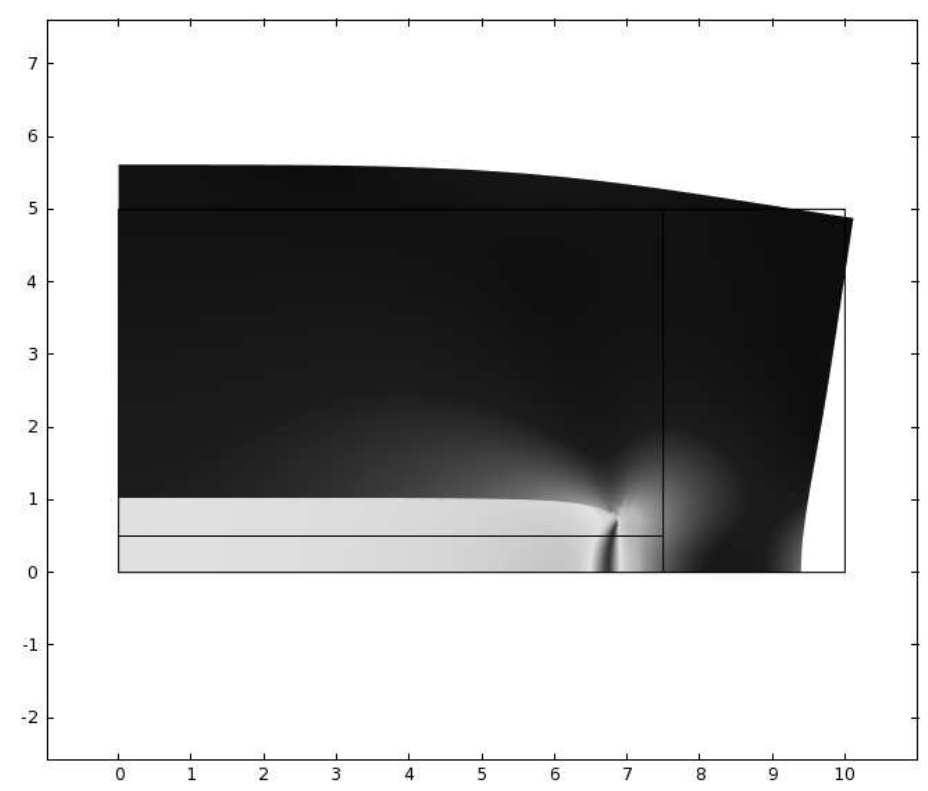

Figura B.2: Deformada do domínio controle obtida via COMSOL.

A comparação dos deslocamentos, na coordenadas $x$, entre alguns nós do domínio é apresentada na Tabela B.1, onde é possível notar valores muito próximos. O desvio médio observado, considerando todos os nós do domínio, é igual a 0, 00010186\% e o Modal Assurance Criterion (MAC) assume valor 0, 999999999998487000, ou seja, praticamente unitário.

No caso dos deslocamentos na coordenada y, a Tabela B.2 apresenta, novamente, valores muito próximos. O desvio médio observado, considerando todos os nós do domínio, é igual a 0,00008464\% e o MAC também assume valor unitário (0, 999999999999113000).

Por último, os potenciais elétricos são apresentados na Tabela B.3. Novamente, valores muito próximos. O desvio médio observado, considerando todos os nós do domínio, é igual a 0,00000330\% e o MAC também assume valor unitário (0, 999999999999879000). 
Tabela B.1: Comparação entre deslocamentos na coordenada $x$ obtidos via AMIGOT e COMSOL.

\begin{tabular}{|c|c|c|c|}
\hline Nó & Comsol $(\mathrm{mm})$ & AMIGOT $(\mathrm{mm})$ & Desvio (\%) \\
\hline 1 & 0,00 & 0,00 & 0,00 \\
\hline 2 & $-4,21 \times 10^{-8}$ & $-4,21 \times 10^{-8}$ & $4,51 \times 10^{-7}$ \\
\hline 3 & $-8,43 \times 10^{-8}$ & $-8,43 \times 10^{-8}$ & $3,68 \times 10^{-7}$ \\
\hline 4 & $-1,27 \times 10^{-7}$ & $-1,27 \times 10^{-7}$ & $3,36 \times 10^{-6}$ \\
\hline 5 & $-1,69 \times 10^{-7}$ & $-1,69 \times 10^{-7}$ & $1,42 \times 10^{-6}$ \\
\hline$\vdots$ & $\vdots$ & $\vdots$ & $\vdots$ \\
\hline 1148 & 0,00 & 0,00 & 0,00 \\
\hline 1149 & $-3,53 \times 10^{-8}$ & $-3,53 \times 10^{-8}$ & $7,93 \times 10^{-7}$ \\
\hline 1150 & $-7,07 \times 10^{-8}$ & $-7,07 \times 10^{-8}$ & $4,53 \times 10^{-7}$ \\
\hline 1151 & $-1,06 \times 10^{-7}$ & $-1,06 \times 10^{-7}$ & $1,29 \times 10^{-6}$ \\
\hline 1152 & $-1,41 \times 10^{-7}$ & $-1,41 \times 10^{-7}$ & $1,97 \times 10^{-6}$ \\
\hline$\vdots$ & $\vdots$ & $\vdots$ & $\vdots$ \\
\hline 2634 & $-3,41 \times 10^{-7}$ & $-3,41 \times 10^{-7}$ & $2,43 \times 10^{-7}$ \\
\hline 2635 & $-3,37 \times 10^{-7}$ & $-3,37 \times 10^{-7}$ & $7,64 \times 10^{-7}$ \\
\hline 2636 & $-3,31 \times 10^{-7}$ & $-3,31 \times 10^{-7}$ & $7,54 \times 10^{-7}$ \\
\hline$\vdots$ & $\vdots$ & $\vdots$ & $\vdots$ \\
\hline 3317 & $7,02 \times 10^{-7}$ & $7,02 \times 10^{-7}$ & $3,27 \times 10^{-8}$ \\
\hline 3318 & $7,02 \times 10^{-7}$ & $7,02 \times 10^{-7}$ & $3,91 \times 10^{-7}$ \\
\hline 3319 & $7,02 \times 10^{-7}$ & $7,02 \times 10^{-7}$ & $6,72 \times 10^{-7}$ \\
\hline 3320 & $7,02 \times 10^{-7}$ & $7,02 \times 10^{-7}$ & $7,83 \times 10^{-8}$ \\
\hline 3321 & $7,02 \times 10^{-7}$ & $7,02 \times 10^{-7}$ & $2,56 \times 10^{-7}$ \\
\hline
\end{tabular}


Tabela B.2: Comparação entre deslocamentos na coordenada $y$ obtidos via AMIGOT e COMSOL.

\begin{tabular}{|c|c|c|c|}
\hline Nó & Comsol $(m m)$ & AmigOT $(\mathrm{mm})$ & Desvio (\%) \\
\hline 1 & 0,00 & 0,00 & 0,00 \\
\hline$\vdots$ & $\vdots$ & $\vdots$ & $\vdots$ \\
\hline 189 & $1,58 \times 10^{-6}$ & $1,58 \times 10^{-6}$ & $7,16 \times 10^{-7}$ \\
\hline 190 & $1,58 \times 10^{-6}$ & $1,58 \times 10^{-6}$ & $1,92 \times 10^{-6}$ \\
\hline 191 & $1,58 \times 10^{-6}$ & $1,58 \times 10^{-6}$ & $1,29 \times 10^{-6}$ \\
\hline 192 & $1,58 \times 10^{-6}$ & $1,58 \times 10^{-6}$ & $1,50 \times 10^{-6}$ \\
\hline 193 & $1,57 \times 10^{-6}$ & $1,57 \times 10^{-6}$ & $8,78 \times 10^{-7}$ \\
\hline$\vdots$ & $\vdots$ & $\vdots$ & $\vdots$ \\
\hline 961 & $2,92 \times 10^{-6}$ & $2,92 \times 10^{-6}$ & $5,91 \times 10^{-7}$ \\
\hline 962 & $2,85 \times 10^{-6}$ & $2,85 \times 10^{-6}$ & $6,80 \times 10^{-7}$ \\
\hline 963 & $2,78 \times 10^{-6}$ & $2,78 \times 10^{-6}$ & $1,69 \times 10^{-6}$ \\
\hline 964 & $2,70 \times 10^{-6}$ & $2,70 \times 10^{-6}$ & $7,83 \times 10^{-7}$ \\
\hline$\vdots$ & $\vdots$ & $\vdots$ & $\vdots$ \\
\hline 2210 & $3,57 \times 10^{-6}$ & $3,57 \times 10^{-6}$ & $3,55 \times 10^{-7}$ \\
\hline 2211 & $3,57 \times 10^{-6}$ & $3,57 \times 10^{-6}$ & $1,31 \times 10^{-6}$ \\
\hline 2212 & $3,56 \times 10^{-6}$ & $3,56 \times 10^{-6}$ & $6,59 \times 10^{-8}$ \\
\hline 2213 & $3,56 \times 10^{-6}$ & $3,56 \times 10^{-6}$ & $1,37 \times 10^{-6}$ \\
\hline 2214 & $3,55 \times 10^{-6}$ & $3,55 \times 10^{-6}$ & $8,65 \times 10^{-7}$ \\
\hline$\vdots$ & $\vdots$ & $\vdots$ & $\vdots$ \\
\hline 3319 & $-5,00 \times 10^{-7}$ & $-5,00 \times 10^{-7}$ & $8,74 \times 10^{-7}$ \\
\hline 3320 & $-6,14 \times 10^{-7}$ & $-6,14 \times 10^{-7}$ & $5,33 \times 10^{-7}$ \\
\hline 3321 & $-7,28 \times 10^{-7}$ & $-7,28 \times 10^{-7}$ & $5,86 \times 10^{-7}$ \\
\hline
\end{tabular}


Tabela B.3: Comparação entre potenciais elétricos obtidos via AMIGOT e COMSOL.

\begin{tabular}{cccc}
\hline Nó & Comsol $(V)$ & AmigOT $(V)$ & Desvio $(\%)$ \\
\hline 111 & $-2,49962722$ & $-2,49963000$ & $1,11309 \times 10^{-6}$ \\
112 & $-2,49959477$ & $-2,49959000$ & $1,90774 \times 10^{-6}$ \\
113 & $-2,49955857$ & $-2,49956000$ & $5,73249 \times 10^{-7}$ \\
114 & $-2,49951799$ & $-2,49952000$ & $8,02518 \times 10^{-7}$ \\
$\vdots$ & $\vdots$ & $\vdots$ & $\vdots$ \\
175 & $-4,99983755$ & $-4,99984000$ & $4,90265 \times 10^{-7}$ \\
176 & $-4,99982737$ & $-4,99983000$ & $5,26303 \times 10^{-7}$ \\
177 & $-4,99981609$ & $-4,99982000$ & $7,82656 \times 10^{-7}$ \\
178 & $-4,99980366$ & $-4,99980000$ & $7,31855 \times 10^{-7}$ \\
179 & $-4,99979003$ & $-4,99979000$ & $6,5548 \times 10^{-9}$ \\
$\vdots$ & $\vdots$ & $\vdots$ & $\vdots$ \\
400 & $-7,49213075$ & $-7,49213000$ & $9,96049 \times 10^{-8}$ \\
401 & $-7,47682809$ & $-7,47683000$ & $2,55656 \times 10^{-7}$ \\
402 & $-7,47761433$ & $-7,47761000$ & $5,78495 \times 10^{-7}$ \\
$\vdots$ & $\vdots$ & $\vdots$ & $\vdots$ \\
3320 & 0,00 & 0,00 & 0 \\
3321 & 0,00 & 0,00 & 0 \\
\hline & & &
\end{tabular}




\section{Apêndice C - Programação Linear Sequencial}

A Programação Linear (PL) é um método matemático utilizado na resolução de problemas lineares de otimização, isto é, que possam ser expressos na seguinte forma:

$$
\begin{aligned}
\text { Maximizar : } & F(\mathbf{d})=\mathbf{A} \cdot \mathbf{d} \\
\mathbf{d} & \\
\text { tal que }: & g_{1}(\mathbf{d})=\mathbf{B} \cdot \mathbf{d} \leq b_{1} \\
& g_{2}(\mathbf{d})=\mathbf{\Gamma} \cdot \mathbf{d} \geq b_{2} \\
& g_{3}(\mathbf{d})=\mathbf{\Delta} \cdot \mathbf{d}=b_{3}
\end{aligned}
$$

onde $\mathbf{A}, \mathbf{B}, \boldsymbol{\Gamma}$ e $\boldsymbol{\Delta}$ são vetores de constantes, $\mathbf{d}$ é o vetor das variáveis de projeto e $b_{1}, b_{2}$ e $b_{3}$ são constantes escalares.

Mesmo com o problema de otimização topológica não sendo linear, é possível fazer tal aproximação por séries de Taylor de primeira ordem, introduzindo um erro dependente da derivada segunda de $\mathbf{d}$. Assim, a função objetivo e as restrições podem ser aproximadas em torno de um ponto $\mathbf{d}^{0}$, sendo que na primeira os termos constantes podem ser desprezados por não exercerem influência sobre a solução.

$$
\begin{aligned}
& \text { Maximizar : } \quad F^{l i n}(\mathbf{d})=\left[\frac{\partial F}{\partial d_{1}} \cdots \frac{\partial F}{\partial d_{n}}\right]_{\mathbf{d}^{0}} \cdot \mathbf{d} \\
& \text { d } \\
& \begin{aligned}
\text { tal que }: & g_{1}^{l i n}(\mathbf{d})=\left[\frac{\partial g_{1}}{\partial d_{1}} \cdots \frac{\partial g_{1}}{\partial d_{n}}\right]_{\mathbf{d}^{0}} \cdot \mathbf{d} \leq b_{1}-\left\{g_{1}-\left[\frac{\partial g_{1}}{\partial d_{1}} \cdots \frac{\partial g_{1}}{\partial d_{n}}\right] \cdot \mathbf{d}\right\}_{\mathbf{d}^{0}} \\
g_{2}^{l i n}(\mathbf{d}) & =\left[\frac{\partial g_{2}}{\partial d_{1}} \cdots \frac{\partial g_{2}}{\partial d_{n}}\right]_{\mathbf{d}^{0}} \cdot \mathbf{d} \geq b_{2}-\left\{g_{2}-\left[\frac{\partial g_{2}}{\partial d_{1}} \cdots \frac{\partial g_{2}}{\partial d_{n}}\right] \cdot \mathbf{d}\right\}_{\mathbf{d}^{0}} \\
g_{3}^{l i n}(\mathbf{d}) & =\left[\frac{\partial g_{3}}{\partial d_{1}} \cdots \frac{\partial g_{3}}{\partial d_{n}}\right]_{\mathbf{d}^{0}} \cdot \mathbf{d}=b_{3}-\left\{g_{3}-\left[\frac{\partial g_{3}}{\partial d_{1}} \cdots \frac{\partial g_{3}}{\partial d_{n}}\right] \cdot \mathbf{d}\right\}_{\mathbf{d}^{0}}
\end{aligned}
\end{aligned}
$$

A solução de problemas lineares, como o apresentado, pode ser obtida via algoritmos como o Simplex (DANTZIG, 1963) ou o Kamarkar (HAFTKA; GURDAL; KAMAT, 1990). Todavia, como essa aproximação apresenta um erro tolerável apenas numa região muito próxima ao ponto $\mathbf{d}^{0}$, é necessário formular e resolver problemas subsequentes em torno de cada ponto $\mathbf{d}^{i+1}$ - Figura C.1. Esse processo iterativo é denominado Programação Linear Sequencial (PLS). 


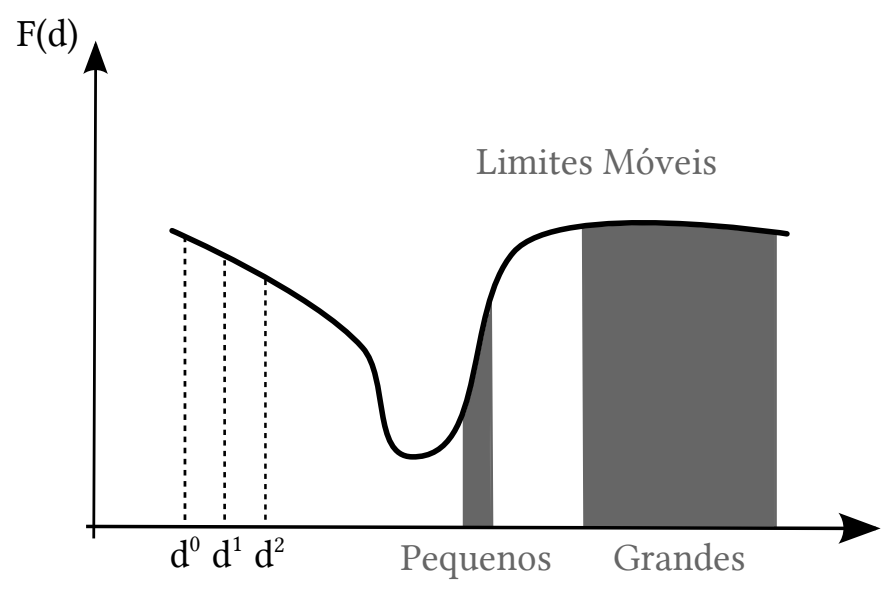

Figura C.1: Programação linear sequencial e os limites móveis.

A distância entre dois pontos de iterações consecutivas é definida por limites móveis. O tamanho dos limites móveis é determinante para a precisão do ponto ótimo e para o número de iterações necessárias até sua obtenção. Se os limites forem muito grandes, acarretarão em um grande erro; se forem muito pequenos, levarão a excessivas iterações. Logo, faz-se necessário variar os limites móveis de acordo com algum critério. As abordagens mais usuais são as dependentes da curvatura da função objetivo, utilizando limites grandes para baixas curvaturas e pequenos para altas - Figura C.1. 


\section{Anexo A - Relações entre Constantes Elásticas}

Relações entre constantes elásticas de materiais isotrópicos (HARTSUIJKER; WELLEMAN, 2001).

\begin{tabular}{cccccc}
\hline & $K$ & $E$ & $G$ & $\nu$ & $M$ \\
\hline$(E, G)$ & $\frac{E G}{3(3 G-E)}$ & $E$ & $G$ & $\frac{E}{2 G}-1$ & $\frac{G(4 G-E)}{3 G-E}$ \\
$(K, G)$ & $K$ & $\frac{9 K G}{3 K+G}$ & $G$ & $\frac{3 K-2 G}{2(3 K+G)}$ & $K+\frac{4 G}{3}$ \\
$(G, \nu)$ & $\frac{2 G(1+\nu)}{3(1-2 \nu)}$ & $2 G(1+\nu)$ & $G$ & $\nu$ & $\frac{2 G(1-\nu)}{1-2 \nu}$ \\
$(E, \nu)$ & $\frac{E}{3(1-2 \nu)}$ & $E$ & $\frac{E}{2(1+\nu)}$ & $\nu$ & $\frac{E(1-\nu)}{(1+\nu)(1-2 \nu)}$ \\
$(K, \nu)$ & $K$ & $3 K(1-2 \nu)$ & $\frac{3 K(1-2 \nu)}{2(1+\nu)}$ & $\nu$ & $\frac{3 K(1-\nu)}{1+\nu}$ \\
$(K, E)$ & $K$ & $E$ & $\frac{3 K E}{9 K-E}$ & $\frac{3 K-E}{6 K}$ & $\frac{3 K(3 K+E)}{9 K-E}$ \\
$(M, G)$ & $M-\frac{4 G}{3}$ & $\frac{G(3 M-4 G)}{M-G}$ & $G$ & $\frac{M-2 G}{2 M-2 G}$ & $M$ \\
\hline
\end{tabular}

onde $K$ denota o módulo de compressibilidade, $E$ o módulo de Young, $G$ o módulo de cisalhamento, $\nu$ o coeficiente de Poisson e $M$ o módulo de onda de pressão. 


\section{Anexo B - Propriedades Teóricas do Material Piezelétrico Utilizado}

Propriedades elásticas do PZT-5A (COMSOL, INC., 2011).

\begin{tabular}{crl}
\hline$\varrho$ & 7,750 & $\mathrm{~g} / \mathrm{cm}^{3}$ \\
$c_{11}$ & 12,0346 & $10^{10} \mathrm{~N} / \mathrm{m}^{2}$ \\
$c_{12}$ & 7,51791 & $10^{10} \mathrm{~N} / \mathrm{m}^{2}$ \\
$c_{13}$ & 7,50901 & $10^{10} \mathrm{~N} / \mathrm{m}^{2}$ \\
$c_{33}$ & 11,0867 & $10^{10} \mathrm{~N} / \mathrm{m}^{2}$ \\
$c_{44}$ & 2,10526 & $10^{10} \mathrm{~N} / \mathrm{m}^{2}$ \\
$e_{13}$ & $-5,35116$ & $\mathrm{C} / \mathrm{m}^{2}$ \\
$e_{33}$ & 15,7835 & $\mathrm{C} / \mathrm{m}^{2}$ \\
$e_{15}$ & 12,2947 & $\mathrm{C} / \mathrm{m}^{2}$ \\
$\varepsilon_{11} / \varepsilon_{0}$ & 1650 & \\
$\varepsilon_{33} / \varepsilon_{0}$ & 1700 & \\
$\varepsilon_{0}$ & 8,85 & $10^{-12} \mathrm{~F} / \mathrm{m}$ \\
\hline
\end{tabular}

